\title{
THE PRECORDIAL FLECTROCARDIOGRAM
}

Frank N. Wilson, M.D., Franklin D. Johnston, M.D., Francis F. Rosenbaum, M.D., Herman Erlanger, M.D., Charias E. Kossmann, M.D., Hans Hecht, M.D., Nelson Cotrim, M.D., Roberto Menezes de Oliveira, M.D., Roberto Scarsi, M.D., and PaUl S. Barker, M.D.

$I^{M}$ NTRODUCTION.-Considerable progress has been made in the standardization of precordial leads, but there is still a great diversity of opinion as to how many leads of this kind should be taken, as to what precordial points should be explored, and as to the proper location for the remote or reference electrode. The multiplicity of leads still in use and still being recommended is the source of much confusion and continues to retard progress in the setting up of adequate normal standards and satisfactory diagnostic criteria applicable to the interpretation of the precordial electrocardiogram. It is not, therefore, surprising that there has been a persistent demand that a single standard method for taking precordial leads be established. Unfortunately, it is not easy to meet this demand for there is no generally accepted method for determining which leads are likely to prove most and which least useful, and there are very great differences of opinion as to whether the decisions required should be made on empirical grounds alone, or, if not, as to what general principles should be taken into consideration.

It seems to us that it would be less difficnlt to solve this problem if there were a more widespread and clearer understanding of the nature of precordial leads, the relations between them and leads of other kinds, the considerations which led to their use, the advantages which have made them popular, and the purposes which they may be expected to serve. We propose, therefore, to discuss the precordial and other special leads which we have used with much satisfaction over a long period. We want to emphasize the principles upon which they are based and to call attention to the kind of information that may be obtained by their use in cases of bundle branch block, ventricular hypertrophy, and myocardial infaretion. It is in these conditions partieularly that the standard limb leads have been found inadequate.

It is true that much of our present knowledge of the human electrocardiogram has been gained by the correlation of electrocardiographic data and clinical or post-mortem observations. Nevertheless, electro-

\footnotetext{
From the Lepartment of Internal Medicine, University of Michigan Medical School. Much of the work upon which this article is based was done with the aid of a grant from the Horace H. Rackham School of Graduate Studies.

This material was presented by Frank N. Wilson at the fffty-third annual meeting of the Association of Life Insurance Medical Dircctors of $\Lambda$ merica held Oct. 21 and 22,1942 , and has been published in the Transactions of that Association (Vol. xxix, pp. 154-251).

Received for publication Oct. 16, 1943.
} 
cardiography can hardly be considered a purely cmpirical science. Its physical and physiological foundations rest upon a large body of critical experimentation dictated by well-constructed hypotheses. These foundations were laid, and the more fundamental principles upon which the interpretation of electrocardiographic records is based were established, before the string galvanometer was introduced into clinical medicine. The more important advances made since that time have come about in the same way as those which preceded them. Few major electrocardiographic problems have been solved by clinical observations alone. For this there are excellent reasons. Our basic knowledge of action currents was acquired by means of direct leads from the tissues under investigation. Unfortunately, it was not possible to study the action currents of the human heart by this method, and indirect leads were necessarily employed for this purpose. The standard limb leads now in universal use have become indispensable, and they are entirely adequate for most of the purposes which clinical electrocardiograms can be expected to serve. They are not, however, capable of furnishing detailed information of the sort obtained when the surface of the exposed heart is explored by means of direct leads. Experiments on animals in which such leads have been employed have consequently continued to play a very important role in the solution of our most difficult electrocardiographic problems. We have not, however, been able hitherto to use all the knowledge acquired in this way to the best possible advantage, because the difference in character between the limb leads used in man and the direct leads used in animal experiments has made it difficult to correlate our elinical and experimental observations. Precordial leads of the type now widely used are much more closely related to direct leads than to the standard limb leads. It is precisely for this reason that they are of such great value. They not only detect lesions which are beyond the range of the standard limb leads, but furnish data that can be directly compared with the data obtained by the use of epicardial leads in animals.

Unipolar Direct Leads.-For the study of eardiac action currents, all kinds of direct leads are not equally satisfactory. In their extensive and detailed study of the course of the excitatory process over the ventricular muscle, Lewis and Rothschild ${ }^{1}$ found that it was difficult or impossible to interpret the records obtained when both electrodes were in contact with the ventricular surface. When leads of this kind were used, the cardiac impulse spreading through the ventricular wall from within outward often reached both electrodes at approximately the same time. The deflections recorded under these circumstances were of complicated and variable form and gave no reliable clue to the time of arrival of the impulse beneath either electrode. When, on the other hand, a small electrode in contact with the epicardium was paired with an electrode on the chest wall, the QRS complex almost invariably 
displayed a large and very abrupt deflection which was easily recognized and was found to coincide with the activation of the muscle in contact with the exploring electrode. The name "intrinsic deflection" was therefore assigned to it. The other deflections of the QRS group, which were attributed to the excitation of muscle more distant from this electrode, were referred to as "extrinsic deflections," but their origin was not studied in detail.

Because of the character of the laws which govern the distribution of electric currents in three-dimensional conductors such as the body, ${ }^{2}$ the magnitude of the potential variations produced by the heartbeat at any point outside the heart itself rapidly diminishes as the distance from the epicardial surface increases. In the course of a cardiac cycle, the variations in potential at a point on the ventricular surface are in the neighborhood of thirty times as large as those displayed by the arms and legs. The deflections of leads from the ventricular surface to some distant part of the body, such as those employed by Lewis and Rothschild, represent the potential variations of the electrode in contact with the heart; the potential variations of the distant electrode are negligible by comparison. It is convenient to use the term "unipolar" to designate leads of this kind in which one electrode is almost completely indifferent, and the term "bipolar" to designate leads like the standard limb leads in which the two electrodes are approximately equidistant from the heart and may, in general, be expected to undergo potential variations of about the same magnitude.

The electrocardiograms reproduced in Fig. 1 were obtained by means of unipolar direct leads from the exposed heart of a dog. Six points on the anterior surface of the ventricle, easy to identify later by their relations to the vesscls or other surface markings, were selected, and, from these, control curves were taken with a small exploring electrode. Points 7,6 , and 8 were on the right ventricle; the first was on the thickest part of the wall near the atrioventricular groove, and the other two were in the central region close to the trabeculated area which overlies the attachment of the anterior papillary muscle. Points 3,1 , and 2 were on the left ventricle; the first was very close to the junction of the free wall and septum, the second near the left margin of the heart, and the third very near the apex. After the control records had been taken, temporary right bundle branch block was produced by pressing upon the right side of the septum above the anterior papillary muscle with the back of a small knife thrust through the wall of the conus arteriosus just below the pulmonary valve. A second set of curves was taken immediately after the knife was withdrawn, and a third set when the right bundle branch had recovered almost completely. Left branch block was then produced by making a cut on the septum just beneath the aortic valve, and a fourth set of records was made. Standard Lead I was taken simultaneously with each direct lead. The intrinsic deflection is easily identified in all of the curves except the first and third from 
point 7 ; it is the sudden downward movement which begins with the peak of $R$. The beginning of this deflection, and therefore the peak of $R$, marks the time of activation of the subepicardial muscle in contact with the exploring electrode. Whether the activation of this muscle and the beginning of the intrinsic deflection coincide exactly or only approximately need not concern us here. It will be noted that, in the leads from the right ventricle, the peak of $R$ fell very late in the QRS interval when right branch block was present and at the very beginning of this interval when left branch block was present. In the leads from points 1 and 2 on the left ventricle these relations are reversed; the peak of $R$ fell late in the QRS interval when left branch block was present and early when right branch block was present. No curve was obtained from point 3 while right branch block was present, for the form of the ventricular deflections of Lead I shows that, when the second tracing was taken from this point, the right bundlc branch had already recovered its conductivity. In the lead from point 3 taken after left branch block was produced, the peak of $R$ was not later than before. This point was very close to the septum, and, in our experience, the intrinsic deflection in leads from this region has seldom been much delayed either by left or by right branch lolock.

When the cardiac mechanism is normal, the intrinsic deflection is always earliest in leads from the trabeculated region, as in the present instance (point 8). It is only a very little later in leads from the central region but considerably later in leads from points near the atrioventricular groove (point $\%$ ) and from points on the conus arteriosus. The earliest intrinsic deflections found on the left ventricle usually occur in leads from the apex (point 2), the latcst, in leads from the lateral part of the free wall toward the base. This region was not studied in the experiment under consideration. The intrinsic deflection is normally considerably later over the surface of the left ventriele as a whole, than over the surface of the right. This difference is attributable to the greater thickness of the left ventricular wall, for in general the latest intrinsic deflections are found where the muscle is thickest.

Origin of the QRS Deflections.--The origin of the more conspicuous and more constant components of the various kinds of ventricular complexes seen in unipolar direct leads is clearly understood. It must be emphasized that, although the excitation of the muscle in contact with the exploring electrode produces a much larger and much more sudden fluctuation in the potential of this electrode than the excitation of any equal mass of muscle at a greater distance from it, every unit of ventricular muscle, without exception, produces action currents which contribute to the form of these complexes. It is obvious that those components of the QRS complex which precede the intrinsic deflection must be attributed to the excitation of muscle which passed into the active state ahead of the subepicardial muscle beneath the exploring electrode, 


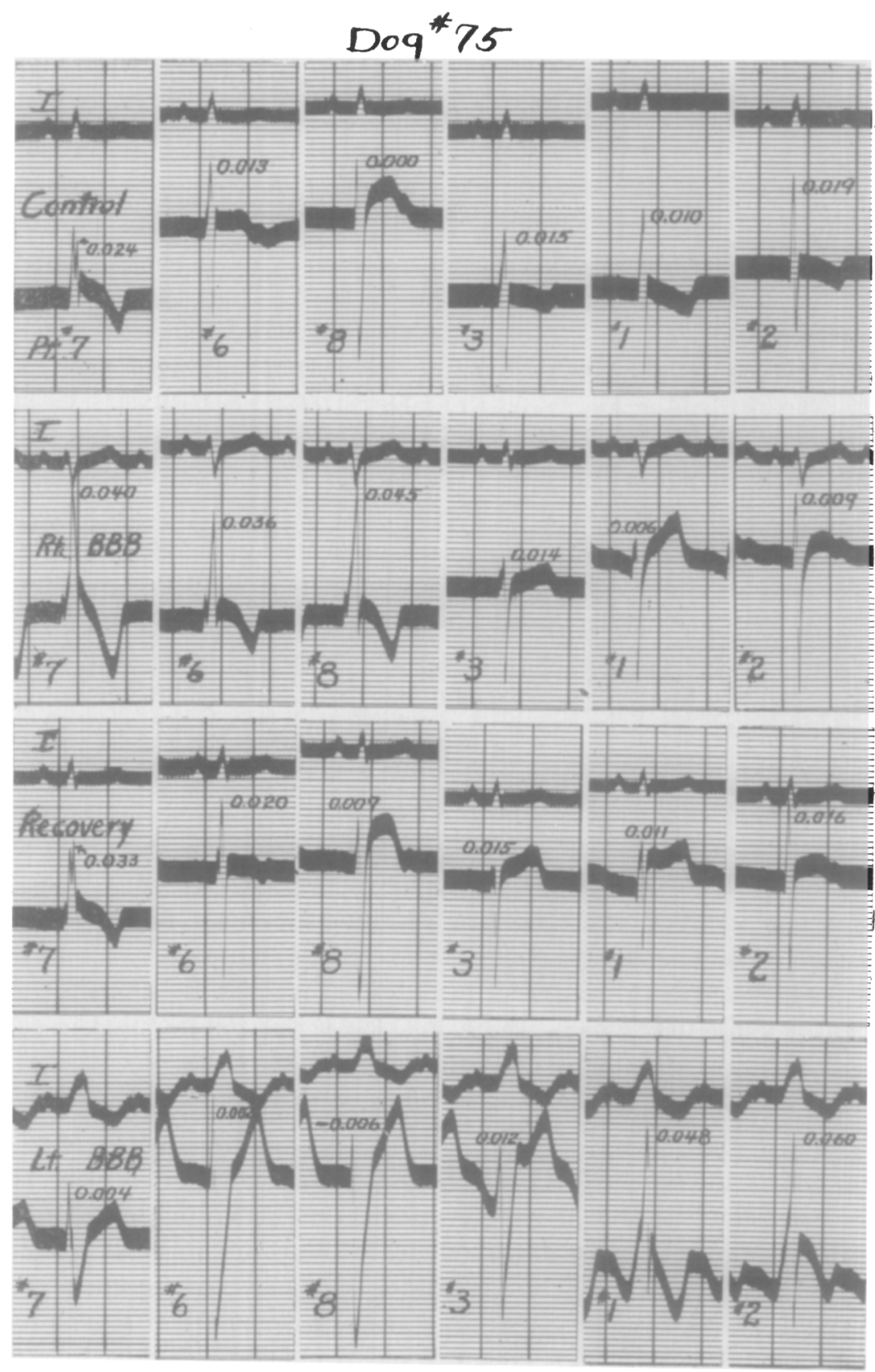

Fig. 1.-Unipolar direct leads from six points on the exposed ventricular surface of a dog's heart. 
and that those components which follow the intrinsic deffection must be ascribed to the excitation of muscle that was still in the resting state when the muscle under the contact became active. The peak of $R$, therefore, separates the QRS components attributable to muscle activated before, from the QRS components attributable to muscle activated after, the cardiac impulse reached the muscle in contact with the exploring electrode.

The resting cardiac muscle fiber is surrounded by a "membrane" which has a very low electrical conductivity. Across this membrane there is an electromotive foree which makes its inner surface strongly negative in comparison with its outer surface. The passage of the excitatory process along the fiber is associated with a sudden and very great increase in the conductivity of the resting membrane and a sudden decrease in the electromotive force (Cole and $\mathrm{Curtis}^{3}$ ); in other words, with the sudden development of a short circuit between the inside and the outside of the fiber. Current flows out of the resting part of the fiber adjacent to the short circuit and re-enters the fiber through the short-circuited region. The effect in the medium outside the fiber is the same as if the cardiac impulse were immediately preceded by the positive pole, and accompanied by the negative pole, of a battery. In other words, we may say that, across the boundary between active and resting muscle, there is an electromotive force which makes the potential of points toward which the excitation wave is advancing positive, and the potential of points which lie behind it negative.

The cardiac impulse spreads through the free walls of the ventricles from within outwards and normally enters the septum from both sides simultaneously. Throughout its course it is traveling away from the ventricular cavities, and these are continuously on the negative side of all the boundaries between active and resting muscle. Consequently, the potential of both cavities is negative throughout the QRS interval ${ }^{4}$ except in those instances in which one side of the septum becomes active before the other, in which case the ventricular cavity on the side activated last almost always displays initial positivity.

Electromotive forces distant from two neighboring points have approximately the same effect upon the potential of both. It is clear, then, that no cardiac electromotive force except that generated by the muscle between them is capable of producing a large difference of potential between two points which are separated solely by the thickness of the ventricular wall. If the potential of the adjacent part of the ventricular cavity is negative, the potential of an epicardial electrode can be positive only while the excitation wave is passing through the part of the ventricular wall upon which it rests. In taking unipolar leads the galvanometer connections are so made that positivity of the exploring electrode produces an upward deflection in the completed record. The $R$ deflection in unipolar leads from the ventricular epicardium of the 
normal heart must, therefore, represent the electrical forces produced by excitation of the muscle between the exploring electrode and the ventricular cavity. The total voltage of thesc forces is, of course, not measured by the height of the $\mathbf{R}$ deflection, but by the difference in potential between the endocardial and the epicardial surfaces of that part of the ventricular wall in contact with the exploring electrode. In bundle branch block most, if not all, of the septal musele is activated from the contralateral side, and the potential of the homolateral cavity is positive until the cardiac impulse has passed through the septum. Under these circumstances, the $\mathbf{R}$ deflection in leads from the outer surface of the delayed ventricle ordinarily displays two peaks. The part of this deflection which precedes the division is due to activation of the septum and represents positivity of the ventricular cavity which is transmitted through the inactive ventricular wall; the part which follows the division is due to activation of the ventricular wall adjacent to the electrode. When this second peak of $\mathrm{R}$ is written, the ventricular cavity is negative.

When the excitatory process reaches the muscle in contact with an epicardial electrode, the boundary between resting and active muscle disappears from that part of the ventricular wall upon which the electrode rests. The potential of the electrode then becomes the potential of the adjacent part of the ventricular cavity. The sudden drop in the potential of the electrode when the electromotive force across the wall beneath it disappears is responsible for the intrinsic deflection. The total length of this deflection is an approximate measure of the voltage across the ventricular wall when the cardiae impulse arrived at the epicardial surface.

If the excitatory process is still spreading through some part of the ventricular wall when the intrinsic deflection occurs, the negativity of the ventricular cavity outlasts this deflection and an $\mathbf{S}$ wave is inseribed. If, on the other hand, the muscle in contact with the exploring electrode is the last ventricular muscle to pass into the active state, the ventricular cavity is at zero potential when the intrinsic deflection is complete and there can be no $\mathrm{S}$ deflection.

If the subendocardial muscle of some part of the ventricular wall passes into the active state earlier than the subendocardial muscle which lies between the exploring electrode and the ventricular cavity, the initial negativity of the cavity is transmitted to this electrode and a $\mathrm{Q}$ deflection occurs. If the cavity is initially positive because of circumstances that we have already mentioned, there can be no $Q$. Since late activation of the subendocardial muscle is likely to be associated with late activation of the subepicardial muscle of the same region, $\mathrm{Q}$ deflections are most common in those leads in which the intrinsic deflection occurs late in the QRS interval, and in which, therefore, $R$ is tall and $S$ is small or absent. 
In summarizing our remarks on the interpretation of the QRS deflections of unipolar direct leads from the ventricular surface, we may point out that the character of these deflections depends mainly upon the time at which the muscle in contact with the exploring electrode becomes active in relation to the beginning and to the nnd of the QRS interval. If this musele becomes active very carly in this interval, the $R$ deflection is narrow and usually small and the $S$ wave broad and nsually deep. If it is activated very late in this interval, $R$ is tall and broad, $\mathrm{S}$ small and narrow, and a $Q$ wave is often present. If it is activated at the very end of the QRS interval, $S$ is absent but there may be a conspicuous $Q$. As the time of activation of the muscle beneath the exploring electrode approaches the beginning of the QRS interval, $\mathrm{R}$ becomes smaller, $\mathrm{S}$ larger, and $\mathrm{Q}$ less frequent. As it approaches the ond of the QRS interval, $R$ becomes larger, $S$ smaller, and $Q$ more frequent. In this discussion we have of course, not considered many of the perplexing details of the QRS complex which may be cneountered in unipolar direct leads as well as in leads of other kinds. Some of these cannot be interpreted satisfactorily until more data are available tham at present.

Unipolar Precordial Leads.--The potential of every extracardiac point inside the body is determined by the potential over the heart's outer surface. The potential variations of every element of the enicardial surface contribute in some measure to the potential variations of an electrode placed upon the precordium. But the magnitude of the contribution made by the potential variations of any given surface element is large if its distance from the electrode is small, and vice versa; in fact, it varies roughly as the inverse cube of this distance. For this reason, the potential variations of a precordial electrode are determined to a very large extent by the potential variations of the elements of ventricular surface nearest it. If all or the groat majority of these elements simultaneously display potential rariations of the same sort, the potential variations of the electrode are of the same character though very much smaller. If, on the other hand, the various elements that are more or less equidistant from the electrode display potential variations of many different kinds, the potential variations of the electrode represent a mixture which may not resemble any of its components very closely.

Since the conditions last mentioned rarely exist, it is almost always possible, by moving an electrode step by step across the precordium, to determine the nature of the potential variations of the various parts of the anterior ventricular surface, provided that the potential variations of the exploring electrode are accurately recorded. In 1929 we began to use multiple precordial leads of this kind to ascertain which of the two ventricles first became active in the classical varieties of bundle branch block. In our first experiments the precordial electrode was paired with an electrode on the left leg. The data obtained in this 
way were not entirely conclusive because the components of the QRS complex due to potential variations of the precordial electrode could not be distinguished with certainty from those due to potential variations of the leg electrode.

In man, the potential variations of the precordium are ordinarily three to five times as large as those of the extremities or of the back. When a precordial electrode is paired with an extremity or dorsal electrode, the general outline of the ventricular complex is determined, therefore, to a very large extent by the potential variations of the former and is much the same regardless of whether the latter is placed upon the right arm, the left arm, the left leg, or the left infrascapular region. But in leads of this kind the potential variations of the reference electrode do exert a very considerable influence upon the details of the form of the ventricular complex and make it impossible to utilize the simple prineiples applicable to the interpretation of the deflections of unipolar direct leads in the analysis of the records obtained.

Many seem to believe that precordial leads are merely additional leads comparable in all respeets to the standard limb leads. The practice of taking a single precordial lead and calling it Lead IV, a practice which we have never followed, is no doubt partly responsible for this entirely unjustifiable attitude. There is no limit to the number of pairs of points on the trunk which might have served as locations for the electrodes if the "cut and try" method had been used to find the additional leads most likely to prove helpful in those situations in which limb leads had been found inadequate. Precordial leads of the kind under consideration were selected for this purpose because observations dictated by theoretical considerations showed that they are in reality semidirect leads from the anterior ventrieular surface, eapable within certain limits of serving the same purposes as direct leads from the ventral wall of the exposed heart. They cannot serve these purposes, however, unless the potential variations of the reference electrode are made negligibly small in eomparison with those of the cxploring electrode. The extent to which the character of the potential variations of the precordial electrode is obscured by the potential variations of the remote electrode determines the degree to which the advantages gained by placing one electrode upon the precordium have been surrendered.

Considerations of this kind led ụs to devise an artificial ground which reduces the potential variations of the remote electrode to a minimum and makes it possible to obtain essentially unipolar leads from the precordium, from the esophagus, from the extremities, and from other parts of the body. This artificial ground consists of a central terminal which is connected through three equal resistances of 5,000 ohms each to eleetrodes on the right arm, left arm, and left leg. ${ }^{5}$ The effect of this device is to balance out those electric forces responsible for differences in potential between these extremities and consequently for the deflec- 
tions of the standard limb leads. It is easily demonstrated that at every instant the potential of the central terminal must be equal to the algebraic mean of the potentials of the three extremity electrodes. On the basis of the assumptions upon which Einthoven's equilateral triangle is founded it has been shown that those components of the heart's resultant electromotive force which are parallel to the plane defined by this triangle have no effect whatever upon the potential of the central terminal. The effects of those components which are perpendicular to this plane cannot be accurately predicted on theoretical grounds. The experiments of Eckey and Fröhlich, ${ }^{6}$ Burger, ${ }^{7}$ Burger and Wuhrmann, ${ }^{8}$ and of others ${ }^{9}$ have, however, demonstrated that the largest potential variations of the central terminal do not ordinarily exceed 0.3 millivolt. The potential of this terminal is, therefore, so nearly constant that for practical purposes we may consider it zero throughout the cardiac cycle. We shall then consider all leads unipolar in which an exploring electrode is paired with an artificial ground of this type.

When we began to use precordial leads in our studies of human and experimental bundle branch block, it soon became evident that the most satisfactory way to obtain semidirect leads from both ventricles was to move the exploring electrode across the precordium, along a line roughly perpendicular to the anterior interventricular sulcus and extending from a point at least as far to the right as the right margin of the sternum to a point well beyond the left border of the heart. We have changed our original method of taking precordial leads only as regards the number and the locations of the points explored, which have undergone minor modifications from time to time. We now take unipolar leads from each of the six points specified by the Committee of the American Heart Association for the Standardization of Precordial Leads. $^{10}$ In accordance with the recommendations of this committee, these leads are designated $V_{1}, V_{2}, V_{3}, V_{4}, V_{5}$, and $V_{6}$. The first of these six points is at the right margin of the sternum in the fourth intercostal space and the second on the left sternal margin at the same level. The remaining points lie on a broken line extending from the second point to the apex beat and thence around the left side of the chest at the apical level. When the apex beat cannot be located, this line is drawn from the second point to the intersection of the left midclavicular line and the fifth intercostal space and is continued around the chest at the level of this intersection. The third point is midway between the second and the left midclavienlar line, the fourth in the midelavicular line, the fifth in the anterior axillary line, and the sixth in the midaxillary line. In addition to these six standard leads, we usually take a unipolar lead from the tip of the ensiform cartilage (Lead $V_{F}$ ) and often take unipolar leads from the posterior axillary line (Lead $V_{7}$ ), from the left infrascapular region $\left(V_{B}\right)$, and from other points.

In the precordial electrocardiograms of normal subjects the ventricular complexes of the leads from the right side of the precordium are 
strikingly similar to those obtained by placing the exploring electrode directly upon the thinner parts of the anterior wall of the dog's right ventricle (Fig. 2). In these complexes the $R$ deflection is small and narrow and $S$ is deep and relatively broad. The peak of $R$ occurs early in the QRS interval and is followed by a large, abrupt downward movement which obviously corresponds to the intrinsie deflection of direct leads. The ventricular complexes of the leads from the left side of the precordium are similar to those seen in direet leads from the thicker parts of the anterior and left lateral walls of the dog's left ventricle.

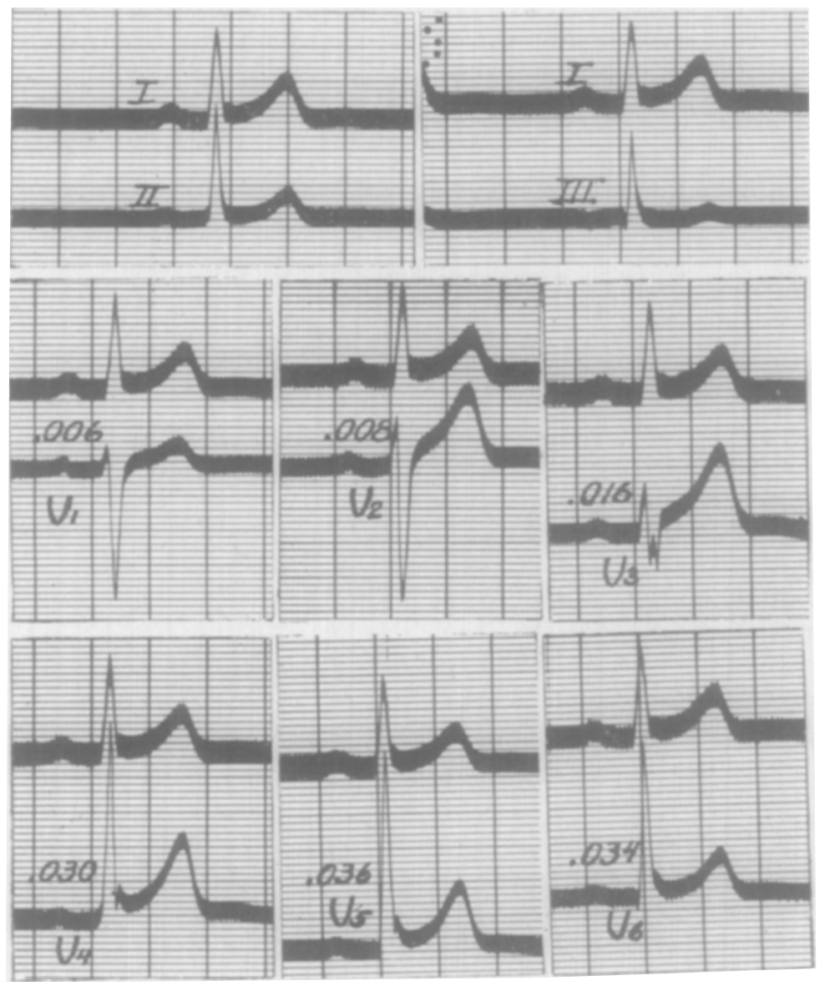

Fig. 2.-The precordial electrocardiogram of a normal subject. The decimals marked on each record give the time of the intrinsic deflection with reference to the beginning of the ORS interval in Lead I, which was taken simultaneously with each precordial lead. QReproduced from an article by Wilson in Stroud's Diagnosis and Treatment of Cardiovascular Disease, by permission of the $\mathrm{F}$. H. Davis Company. Philadelphia.)

The $\mathbf{R}$ component of these complexes is tall and relatively wide and it is often preceded by a $Q$ and followed by an $S$ deflection. The peak of $R$ which marks the onset of the intrinsie downstroke is, on the average, about 0.02 second later in leads from the left side of the precordium than in leads from the right side. ${ }^{11}$ In precordial leads this downstroke is not nearly so sudden as in direct leads and, therefore, is somewhat more difficult to identify with certainty when the contour of the ventricular complex is complicated or unfamiliar. 


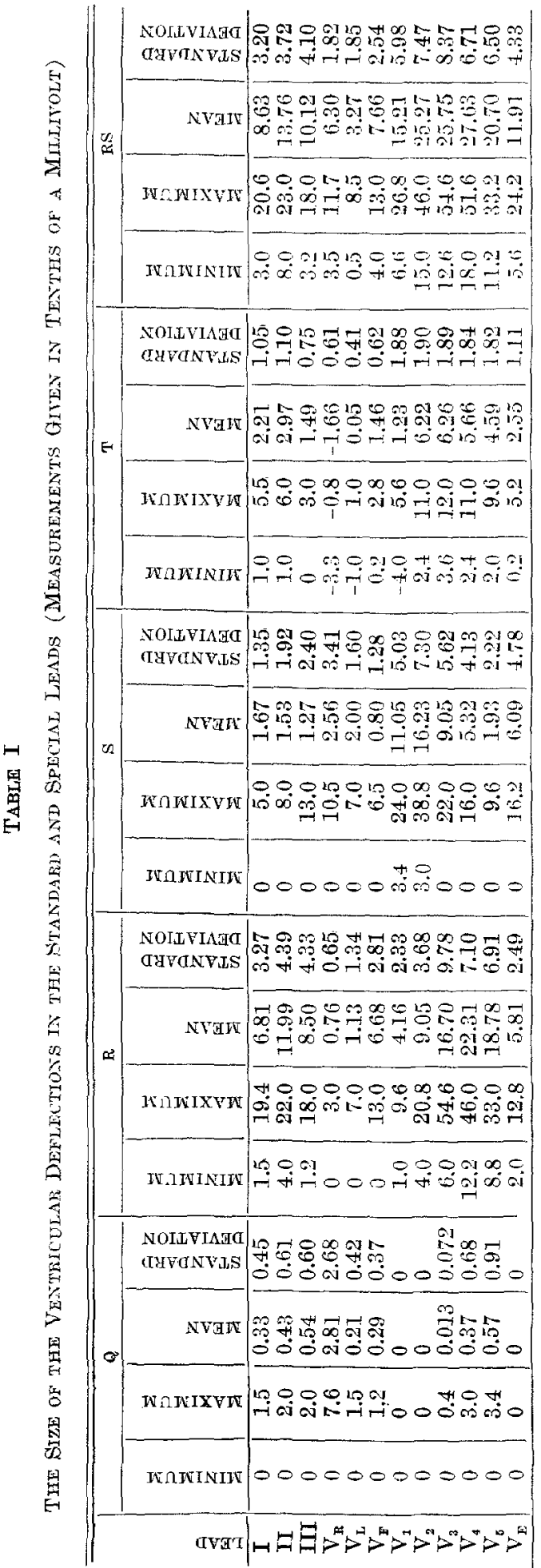


Between those parts of the precordium which yield small $R$ and deep $S$ waves and those which yield QRS complexes of the opposite kind, there is a zone which yields complexes that are intermediate in form or represent a combination of the deflections obtained at points farther to the right and those obtained at points farther to the left. This zone varies from subject to subject, both in location and in width, and is probably not absolutely constant in either particular in one and the same subject. The ventricular complexes of leads from this transitional zone may display notching and other peculiarities of form. They represent a mixture to which the potential variations of those parts of the right ventricle and the potential variations of those parts of the left ventricle which are least distant from the exploring electrode contribute in approximately equal measure.

The minimal, maximal, and mean voltages of $Q, R, S$, and $T$ in precordial Leads $V_{1}, V_{2}, V_{3}, V_{4}, V_{5}$, and $V_{E}$, and in unipolar extremity Leads $V_{R}, V_{L}$, and $V_{F}$, found by Kossmann and Johnston ${ }^{11}$ in their study of thirty normal subjects, are given in Table $\mathrm{I}$, which is reproduced in modified form from their paper.

Unipolar Limb Leads.-Unipolar leads from the right arm $\left(V_{R}\right)$, left $\operatorname{arm}\left(V_{L}\right)$, and left leg $\left(V_{F}\right)$ furnish a valuable means of comparing the potential variations of these extremities with those of the right and left sides of the precordium and, consequently, of establishing relations between the complexes of the standard limb leads and those of the precordial leads. Unipolar limb leads mav be taken by placing the exploring electrode used in taking the precordial leads on each of the extremities in turn or by transferring the wire connected to this electrode to each of the extremily electrodes. When this is done, the deflections obtained are often inconveniently small unless the sensitivity of the electrocardiograph is increased to one and a half or two times its normal value. Goldberger ${ }^{12}$ has introduced a procedure which avoids this difficulty. By breaking the connection between the central terminal and the extremity upon which the exploring electrode has been placed, he increases the size of the deflections obtained by fifty per cent without changing the sensitivity of his apparatus. The standard limb leads and the unipolar limb leads are, of course, directly and simply related. The deflections of any two leads of either set furnish all the data needed to compute the deflections of the other four. The equations which express these relations have been published elsewhere ${ }^{5}$ and need not be repeated here. It may be pointed out here, however, that when the ventricular complexes of Leads I and II are similar in form, the complexes of Lead $V_{R}$ resemble the complexes of these leads turned upside down; that when the complexes of Lead III are the inverse of those of Lead I, the complexes of Lead $V_{L}$ are like those of Lead I; and that when the complexes of Leads II and III are alike, those of Lead $V_{F}$ have the same form. It should also be remembered that at any instant 
the algebraic sum of the potentials of the three extremities is equal to zero.

The Position of the Heart From the Electrocardiographic Standpoint. -The standard and unipolar electrocardiograms of six subjects are reproduced in Fig. 3. Not all of these tracings are normal, but, so far
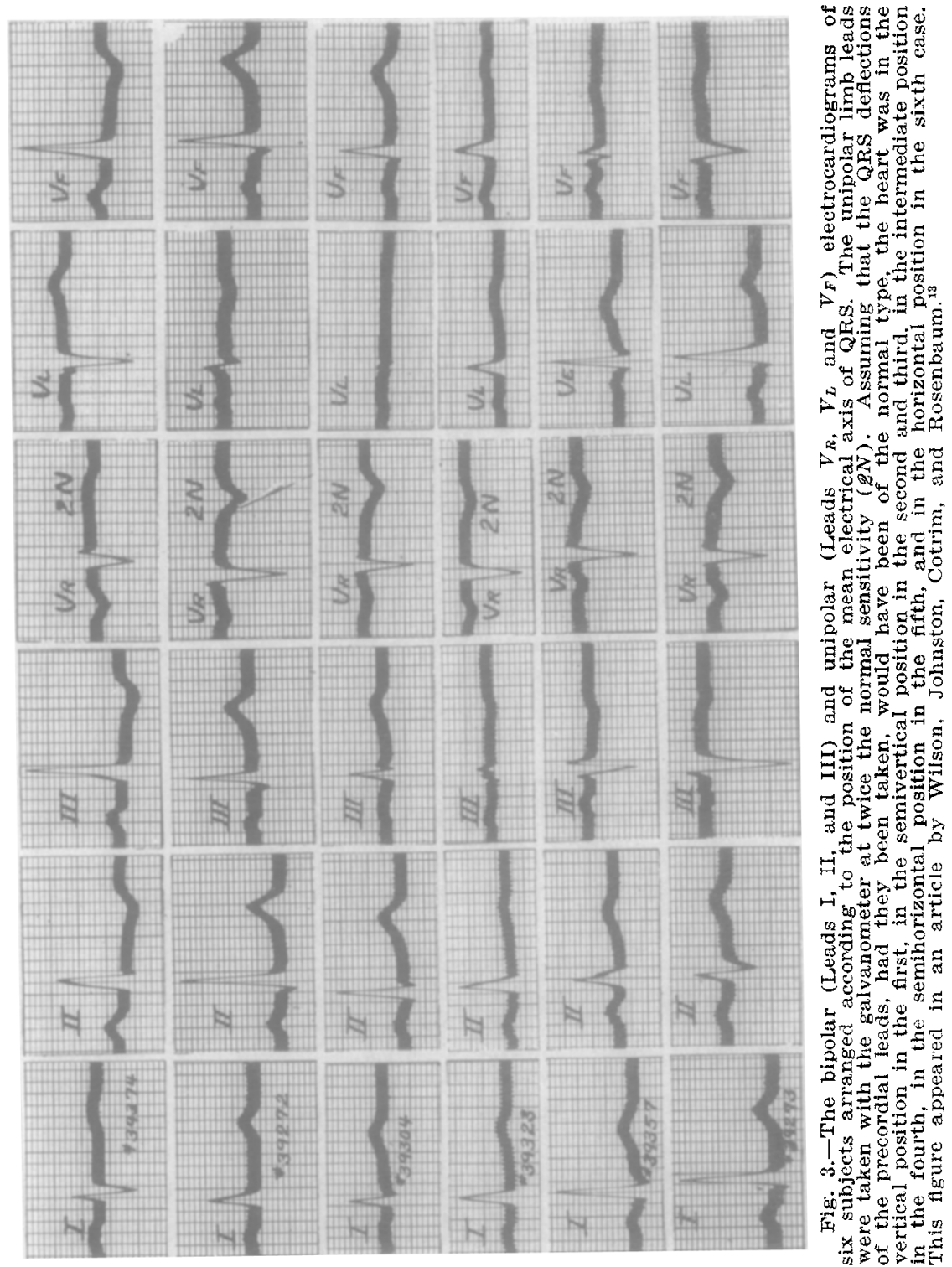

as the QRS complexes are concerned, similar curves could be selected from any collection of normal records. They are arranged in accordance with the position of the mean electrical axis of QRS; the one which displays the greatest degree of right axis deviation is at the top and the one which displays the greatest degree of left axis deviation is 
at the bottom. It will be noted that the potential variations of the right arm $\left(V_{R}\right)$ were always of the same general kind. In some cases, this extremity was slightly positive at the beginning, at the end, or both at the beginning and at the end of the QRS interval, but throughout by far the greater part of this interval it was conspicuously negative in all. The potential variations of the other two extremities varied greatly with the position of the electrical axis. When this axis was shifted to the right, as in the first electrocardiogram, the potential variations of the left $\operatorname{arm}\left(V_{L}\right)$ were like those which occur over the anterior surface of the dog's right ventricle and over the right side of the precordium in normal subjects. The potential variations of the left leg $\left(V_{F}\right)$, on the other hand, resembled those which occur over the anterolateral surface of the dog's left ventricle and over the left side of the precordium in normal subjects. When the electrieal axis was shifted to the left, as in the last electrocardiogram, just the reverse was the case; the potential variations of the left arm resembled those normally found on the left side of the precordium, and the potential variations of the leit leg, those normally found on the right side..$^{13}$

When the heart is normal, the precordial electrocardiogram has essentially the same form regardless of whether the limb leads display right axis deviation, no axis deviation, or left axis deviation. It is evident, therefore, that, in normal subjects with right axis deviation, the potential variations of the right ventricular surface are transmitted to the left arm and the potential variations of the left ventricular surface to the left leg, whereas, in normal subjects with left axis deviation, the potential variations of the right ventricular surface are transmitted to the left leg and the potential variations of the left ventricular surface to the left arm. These differences in the magnitudes of the contributions made by the right and left ventricular surfaces to the potential variations of arm and leg can hardly depend upon factors lying within the heart itself; they must be due to variations in the spatial relations between the surfaces of the two ventricles and the attachments of the extremities to the trunk. When the electrocardiogram is normal, there is an obvious correlation between the inclination of the mean electrical axis of QRS and the position of the heart. When the long axis of the heart is nearly vertical, right axis deviation is usually present, and when the long axis of the heart is nearly horizontal, left axis deviation is usually present. We are justified, therefore, in using the terms "vertical" and "horizontal" in defining the position of the heart from the purely electrocardiographic standpoint. So far as the normal heart is concerned, the first five of the six cardiac positions deseribed below could be defined equally well in terms of the position of the electrical axis, but, if that were done, the definitions could not be applied when the heart was abnormal.

From the purely electrocardiographic standpoint, we may then define the following six positions of the heart (Fig. 3). 
Vertical Position.--

a. The ventricular complexes of I ead $V_{L}$, resemble those of Leads $V_{1}$ and $V_{2}$.

b. The ventricular complexes of Lead $V_{F}$ resemble those of Leads $V_{5}$ and $V_{6}$.

Semivertical Positions.-...

a. The ventricular complexes of Lead $V_{F}$ resemble the ventricular complexes of Leads $V_{5}$ and $V_{6}$.

b. The QRS deflections of Lead $V_{L}$ are small.

Intermediate Position.-

a. The ventricular complexes of Leads $V_{L}$ and $V_{F}$ are similar in form and size and like those of Leads $V_{\tilde{a}}$ and $V_{6}$.

Semihorizontal Position.-

a. The ventricular complexes of Thead $V_{L}$ resemble those of Leads $V_{5}$ and $V_{6}$.

b. The QRS deffections of Lead $V_{F}$ are small.

Horizontal Position.-...

a. The ventricular complexes of Lead $V_{L_{L}}$ resemble those of Leads $V_{5}$ and $V_{6}$.

b. The ventricular complexes of Lead $V_{F}$ resemble those of Iseads $V_{1}$ and $V_{2}$.

Indeterminate Position.--There is no obvious relationship between the ventricular complexes of the limb leads and those of the precordial leads.

As long ago as 1910, Kraus and Nicolai ${ }^{14}$ asserted that the deflections obtained by leading from the surface of the body are very similar to those that would be obtained by leading from those parts of the heart's surface beneath and nearest the electrodes. They based this opinion upon the principles that govern the distribution of electric currents in volume conductors. As regards precordial leads, we reached a similar conclusion long before we knew that others had held this view, and we have demonstrated that there is a very close relation between the potential variations of a precordial clectrode and the potential variations of the underlying ventricular surface. There is considerable evidence of a less direct kind that the potential variations of the right arm are similar to the potential variations of those parts of the heart's surface that are nearest the right shoulder; that the potential variations of the left arm resemble the potential variations of those parts of the heart's surface that are nearest the left shoulder; and that the potential variations of the left leg are like the potential variations of the heart's diaphragmatic surface.

We have already mentioned that the potential of the right arm is ordinarily negative throughout the greater part of the QRS interval. The reason lies in the relation of the right shoulder to the great valvular 
orifices at the base of the heart. The negativity of the ventricular cavities is transmitted through these orifices to the adjacent parts of the body, including the right shoulder and the right arm. The potential variations of this extremity do not represent the potential variations of the ventricular cavities in pure form, of course, but mixed with the potential variations of other regions and particularly with those of the surface of the right ventricle. Over the thinner parts of the free wall of this chamber, the potential is normally weakly positive at the very beginning of the QRS interval, but during the rest of this interval it does not differ from that of the ventricular cavity. It is frequently impossible, therefore, to say whether the base of the heart or the right ventricular surface contributed most to the potential variations of the right arm. The same is sometimes true with reference to the potential variations of the left arm. It is well known that when the heart assumes a more vertical position or turns about its long axis in the direction required to bring the left ventricle below the right, the potential variations of the left leg become more like those of the left ventricular surface and less like those of the right ventricular surface, while the potential variations of the left arm change in the opposite way. When the heart assumes a more horizontal position or twists about its long axis in the direction required to bring the right ventricle below the left, the effects upon the potential variations of the left arm and left leg are the reverse of those described.

It is also true that, in myocardial infaretion, the potential variations that occur at the epicardial surface of the infaret are transmitted to the left arm when the anterolateral wall of the left ventricle is involved and to the left leg when the diaphragmatic wall is involved.

But although the anatomical position of the heart and its electrocardiographic position are very closely related, there are reasons for believing that the correlation between the two cannot be perfect. It is evident that those parts of the right ventricular surface which play the most important role in determining the potential variations of the right side of the precordium are not identical with those parts of this surface which play the most important role in determining the potential variations of the left arm or left leg, as the case may be. A similar statement may be made with reference to those parts of the left ventricular surface which contribute most to the potential variations of the left side of the precordium. Significant relations between the ventricular complexes of the precordial leads, and the ventricular complexes of the unipolar limb leads of the kind upon which the determination of the electrocardiographic position of the heart is based, can be expected to occur, therefore, only when potential variations of one kind take place simultaneously over a large part of the right ventricular surface and potential variations of another kind take place simultaneously over a large part of the left ventricular surface. This is likely to happen 
when the heart is normal, in right and in left bundle branch block, and in preponderant hypertrophy of the right or left ventriele. It is much less likely to happen when there are lesions which give rise to local modifications of the potential variations at the surface of the heart or

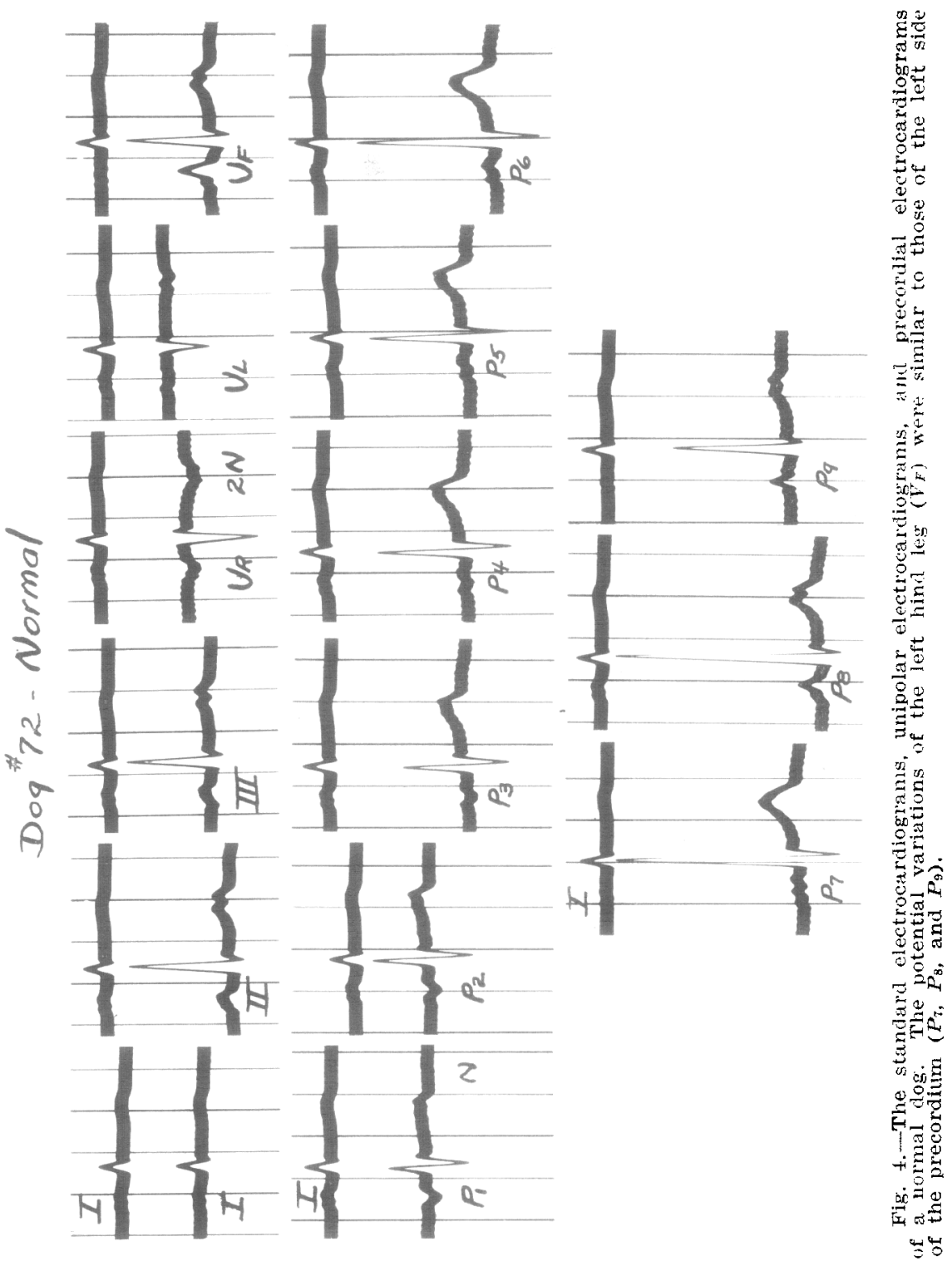

which have opposite effects upon the potential variations of different parts of the surface of the same ventricle. For this reason and because the potential variations of the extremities often represent complicated mixtures, it is often impossible in cases of myocardial disease to establish any relation between the potential variations of the procordium and the potential variations of the extremities. 
It should be noted that the electrocardiographic position of the heart may change even though the anatomical position remains the same. This is tme because the former is determined not only by the orientation of the surfaces of the two ventricles, but also by the distribution of potential variations of various kinds on each of them. In bundle branch block, for example, the regions in which the same kind of potential variations occur at the same time are not exactly the same in extent and configuration as when the heart is normal. Consequently, the onset of bundle branch block may, although it usually does not, alter the position of the heart from the electrocardiographic standpoint.

The Precordial Electrocardiograms of Dog and Man.- The electrocardiograms reproduced in Fig. 4 represent observations made in the course of an experiment upon a normal dog. Standard limb leads, unipolar limb leads, and nine unipolar precordial leads were taken. The first precordial lead was from a point $4 \mathrm{~cm}$., and the sceond from a point $2 \mathrm{~cm}$., to the right of the midline; the other precordial leads were taken by moving the exploring electrode across the precordium in a baseapex direction and were from points $2 \mathrm{~cm}$. apart. The precordial curves closely resemble the normal human curves obtained by the same method. The potential variations of the two forelegs were similar; both extremities were negative throughout the greater part of the QRS interval (Leads $V_{R}$ and $V_{L}$ ). The ventricular complexes of Lead $V_{F}$ are like those obtained from the left side of the precordium (Leads $P_{5}, P_{6}, P_{7}$, $P_{8}$, and $P_{0}$ ) and the deflections of Lead $V_{L}$ are smaller than those of the other unipolar limb leads. The heart, then, was in the semivertical position.

The electrocardiograms shown in Fig. 5 are reproduced to demonstrate that there are no essential differences between the precordial electrocardiograms obtained in canine bundle branch block and those obtained in human bundle branch block. The curves of the first set represent left branch block in man; those of the second set left branch block in the dog. In both cases the ventricular complexes of the leads from the right side of the precordium display small $R$ and broad $S$ deflections, indicating that the excitatory process arrived at the surface of the right ventricle very early in the QRS interval. The ventricular complexes of the leads from the left side of the precordium, on the other hand, are dominated by broad notehed $R$ waves of the kind secn in direct leads from the anterolateral wall of the left ventricle after section of the left branch of the His bundle. The curves of the third set represent human right branch block, and those of the fourth set, canine right branch block. Here the complexes of the leads from the right side of the precordium display a small initial $R$ and a tall final $R$ deflection and are similar to those obtained by leading directly from the surface of the right ventricle after the right branch of the His bundle has been cut. The ventricular complexes of the leads from the left side of the 

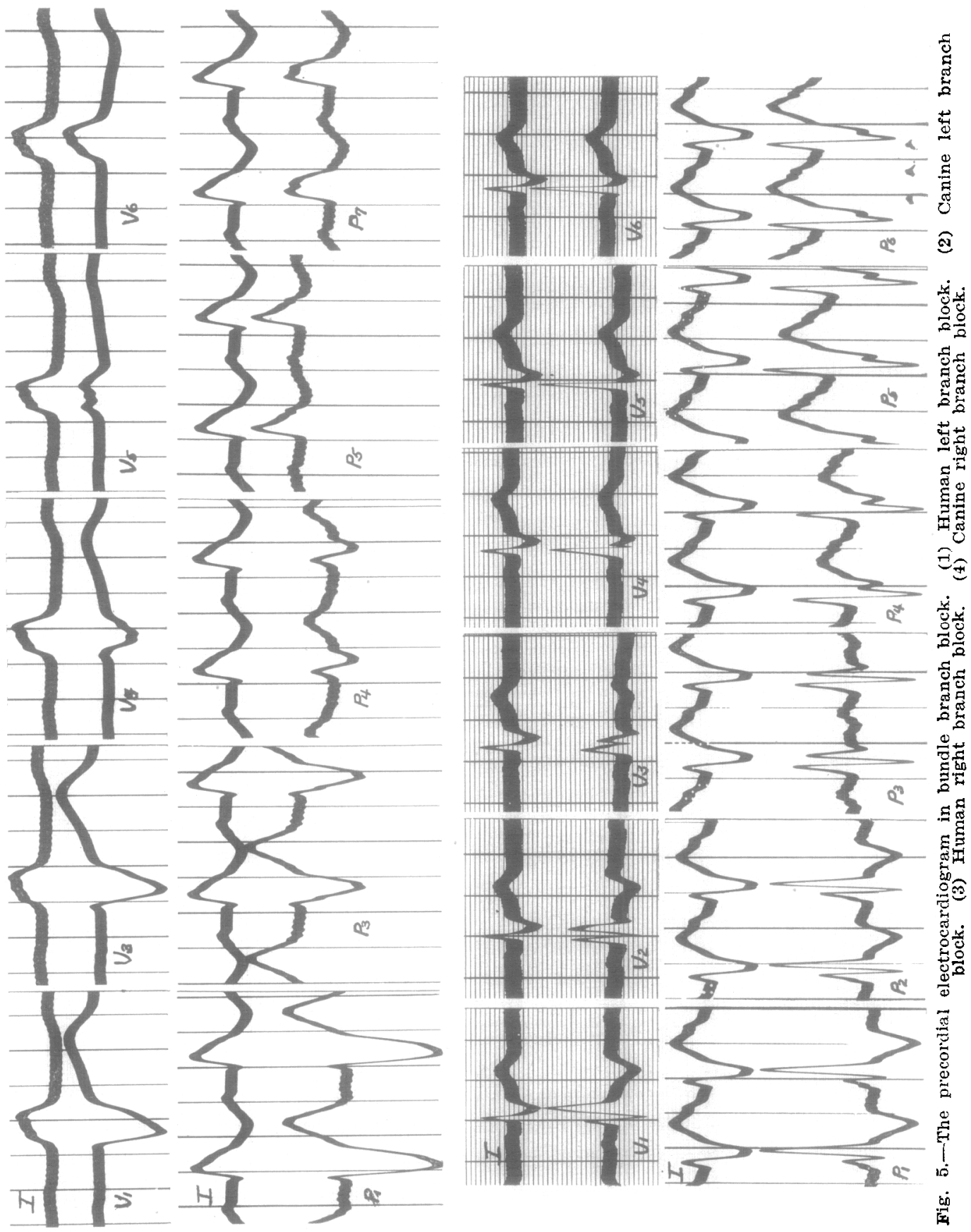
precordium show narrow $R$ and broad notched $\mathbf{S}$ deflections, indicating early activation of the left ventricular surface. It is evident that, if precordial leads instead of limb leads had been used in the early studies of experimental and clinical bundle branch block, human left branch block could never have been mistaken for right branch block over a period of nearly twenty years. The confusion which prevailed over this long period arose because the dog's heart is almost always in the semivertical position, whereas, in patients with arterioselerotic heart disease, the heart is usually in either the semihorizontal or the horizontal position. Since the relations between the surfaces of the two ventricles and the attachments of the extremities to the trunk are not constant in man, the differentiation of human right, from human left, bundle branch block, on the basis of the data furnished by limb leads alone, is sometimes impossible.

Comparison of Precordial and Direct Leads.--The similarities and differences between unipolar precordial leads and unipolar direct leads from the anterior surface of the heart can be appreciated only when these two kinds of leads are directly compared. In a number of experiments on dogs, we cut one of the branches of the His bundle under aseptic conditions, restored the chest, and allowed the animal to recover completely. Approximately one month after this operation, unipolar precordial leads were taken with the chest intact. The heart was then exposed by splitting the sternum and the potential variations of numerous points on its anterior surface were recorded.

The curves shown in Fig. 6 are from an experiment in which the right branch of the His bundle was cut twenty-six days before the electrocardiographic studies were made. Six of the precordial electrocardiograms are reproduced $\left(P_{1}, P_{3}, P_{4}, P_{5}, P_{6}\right.$, and $\left.P_{8}\right)$. Below these are the records of the potential variations of three points on the right ventricle (points 4, 5, and 6), one point near the interventricular suleus (point 3), and two points on the surface of the left ventricle (points 1 and 2). Point 4 was on the conus, point 5 near the atrioventricular groove, point 6 in the central region, and points 2 and 1 on the left horder above the apex.

The first three curves of the bottom row were obtained before the chest was opened by thrusting a sharp exploring electrode, insulated except at the extreme tip, through the chest wall on the right side of the precordium. The first curve was taken when the tip of the electrode was just beneath the skin, the second when it touched the heart and yielded a monophasic response, and the third when it was in the cavity of the right ventricle. The last three curves were obtained in the same way except that the sharp electrode was thrust through the chest wall at a point just inside the apex beat, consequently entering the cavity of the left ventricle. It will be noted that the cavity of this chamber was negative throughout the QRS interval. The potential variations of the 
cavity of the right ventricle were not of the usual torm inasmuch as the positivity of this chamber, due to the spread of the impulse through the septum from left to right, did not oceur at the very beginning of the $Q R S$ interval as it ordinarily does. The $\mathbf{R}$ wave to which it gave rise is preceded, therefore, by a $Q$ deflection.

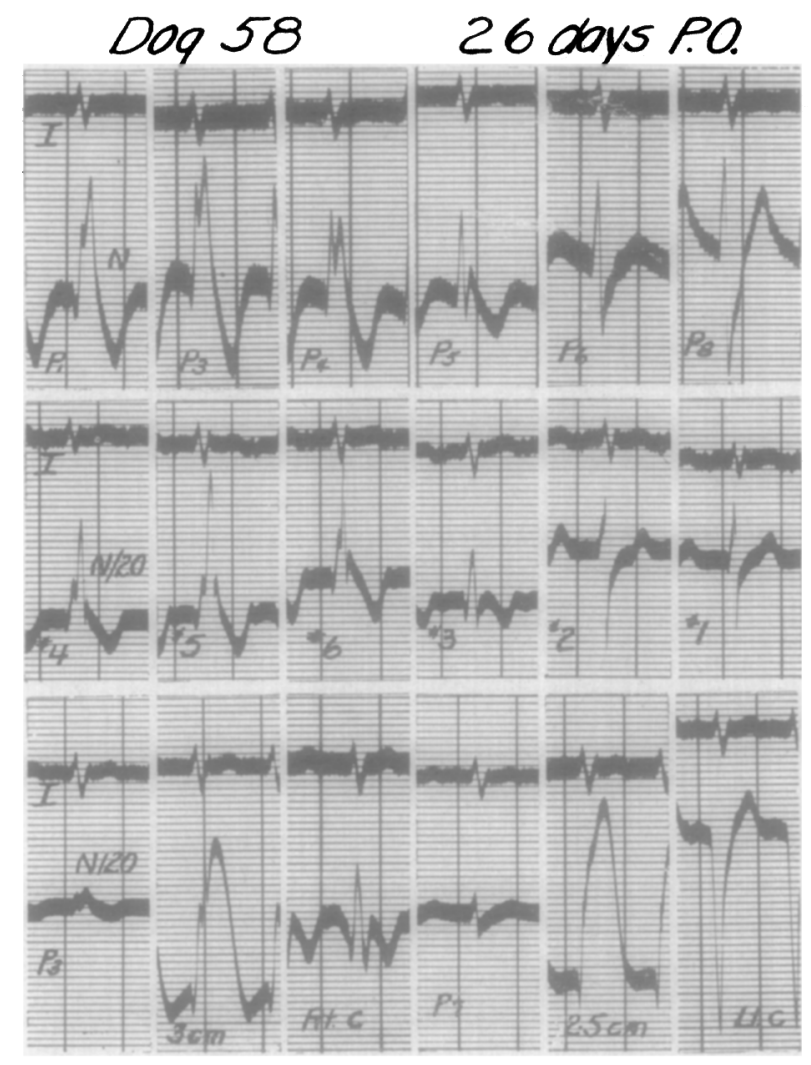
Fig. 6.-Precordial and direct leads in canine right branch block. (1) The pre-
cordial electrocardiogram. (2) Direct leads from three points on the right and three
points on the left ventricle. (3) $l t .6$, Hotential variations of the cavity of the right ventricle. $L t$. $C$, Potential variations of the cavity of the left ventricle.

The curves shown in Fig. 7 are from an experiment in which the left branch of the His bundle was cut twenty-seven days before the electrocardiographic study was made. Eight precordial electrocardiograms are reproduced. Below these are records of the potential variations of the cavity of the right ventricle (12c), three points on the right ventricle $(8,3,5)$, three points on the left ventricle $(6,1,7)$, and the cavity of the left ventricle $(13 \mathrm{c})$. Point 8 was near the atrioventricular groove, point 3 in the upper part of the central region, point 5 close to the trabeculated region, point 6 near the apex, and points 1 and $r$ on the left border well above the apex. It will be noted that the cavity of the right ventricle was ncgative throughout the QRS interval while that of the left ventricle displayed initial positivity. 
The similarity between the potential variations of the exposed surface of the right ventricle and the potential variations of the right side of the precordium, and between the potential variations of the surface of the left ventricle and those of the left side of the prccordium in these two experiments requires no comment. We may point out, however, that the first peak of the bifid $\mathrm{R}$ waves often recorded over the delayed ventricle oceurs when the cavity of that ventricle is positive, whereas the second peak, which marks the beginning of the intrinsic deflection, occurs when the cavity is negative. In those cases in which the peak of this broad $R$ wave is not divided, the first peak is usually represented by a shoulder on its ascending limb.

Dog 64
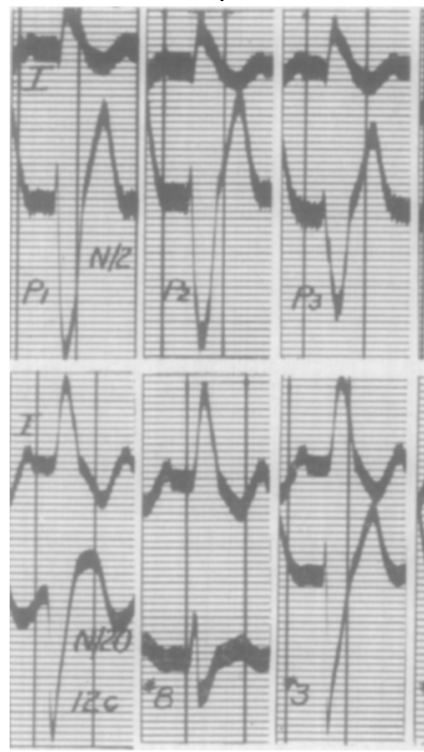

27 days P. O.
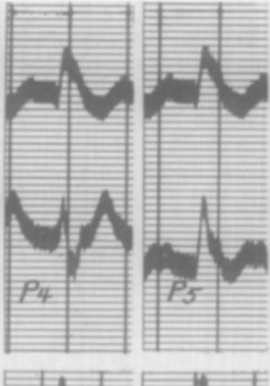
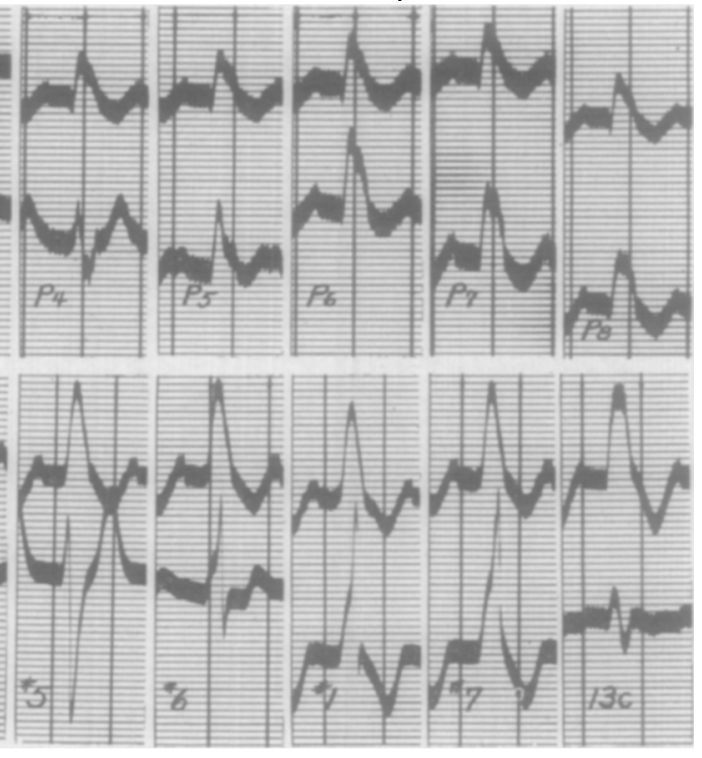

Fig. 7.-Precordial and direct leads in canine left hranch block. (1) The precordial electrocardiogram. (2) The potential variations of three points $(8,3,5)$ on the right, and three points $(6,1, \gamma)$ on the left ventricle. Potential variations of the cavity of the right $(12 c)$ and of the cavity of the left $(13 c)$ ventricle.

II uman Bundle Branch Block.-There can be no reasonable doubt that, in man as in the dog, the potential variations of the right side of the precordium (Leads $V_{1}$ and $V_{2}$ ) ordinarily resemble the potential variations of the anterior surface of the right ventricle, while the potential variations of the left side of the precordium (Leads $V_{5}$ and $V_{6}$ ) ordinarily resemble the potential variations of the anterolateral surface of the left ventricle.

The electrocardiograms shown in Fig. 8 are those of a man who was examined both before and after he developed left bundle branch block. The tracings of March 28, 1940, display slight left axis deviation and flat $\mathrm{T}$ waves in Lead II; the QRS interval measures about 0.09 second. 
On Dec. 9, 1940, left branch block was present. On this occasion, unipolar limb leads, unipolar precordial leads, and unipolar leads from the left infrascapular region were taken. The potential variations of the left arm $\left(V_{L}\right)$ were similar to those of the left side of the precordium and the left infrascapular region, whereas the potential variations of the left leg were like those of the right side of the precordium in general outline. The heart was then in the horizontal position.

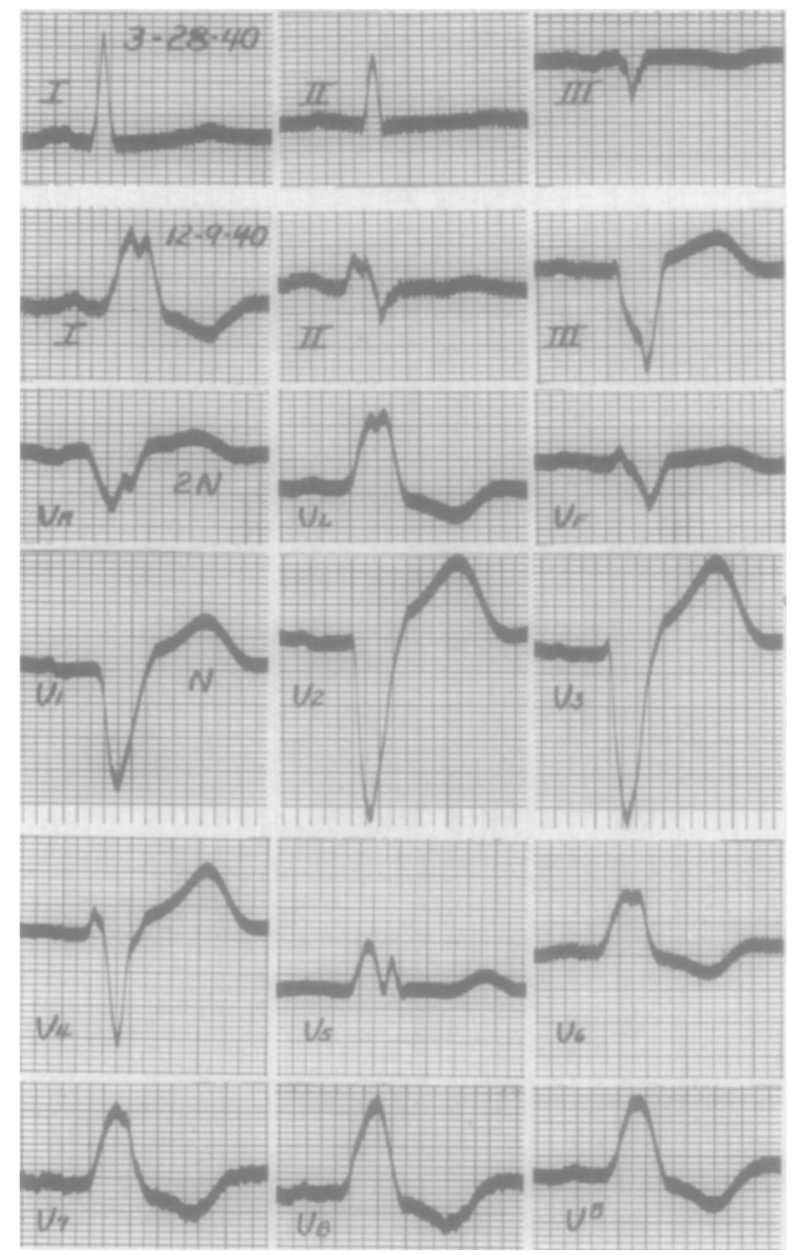

Fig. 8.- Left bundle branch block; heart in the horizontal position.

The electrocardiograms shown in Fig. 9 are those of a tall thin man who was examined for the first time on Feb. 3, 1937, when his electrocardiogram was normal. There was no deviation of the mean electrical axis at this time. On April 14, 1940, left hundle branch block was present and there were broad notched $R$ deflections in Leads I and II and broad $S$ deflections in Lead III. The precordial electrocardiograms of that date differ in no essential particular from those reproduced and 
the heart was evidently in the semihorizontal position. On Nov. 27, 1940, and on Dec. 6, 1940, the ventricular complexes of the standard limb leads were of the concordant type. The unipolar leads taken on the latter date show that the potential variations of the left leg were like those of the left side of the precordium and the left infrascapular region. The potential variations of the left arm were small on both oecasions. On these two days the heart was in the semivertical position.

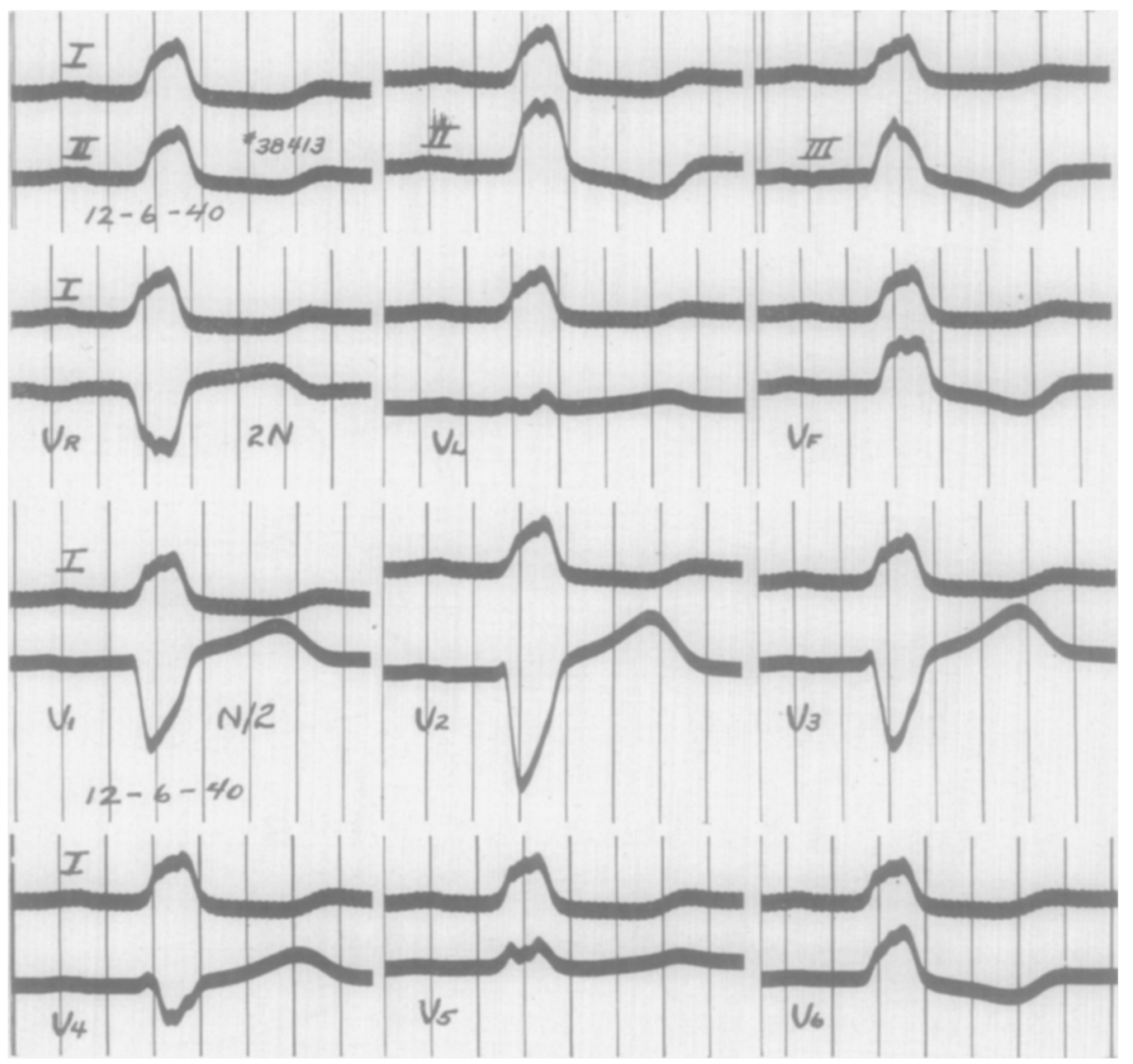

Fig. 9.-Left bundle branch block; heart in the semivertical position.

The patient whose electrocardiograms were reproduced in Fig. 10 was under observation from Feb. 26, 1941, until March 22, 1941. The ventricular complexes of the standard limb leads were quite variable in form. The curves taken on February 28 display discordant ventrieular complexes of the kind ascribed to bundle branch block of the rare type. Those taken on March 14 show small bizarre QRS deflections in Lead I and diphasic QRS complexes with broad initial $R$ waves in Leads II and III. In the tracings taken on Feb. 26, March 13, March 18, and March 26 , the ventricular complexes are intermediate in form between the two types reproduced. Precordial electrocardiograms were taken on March 


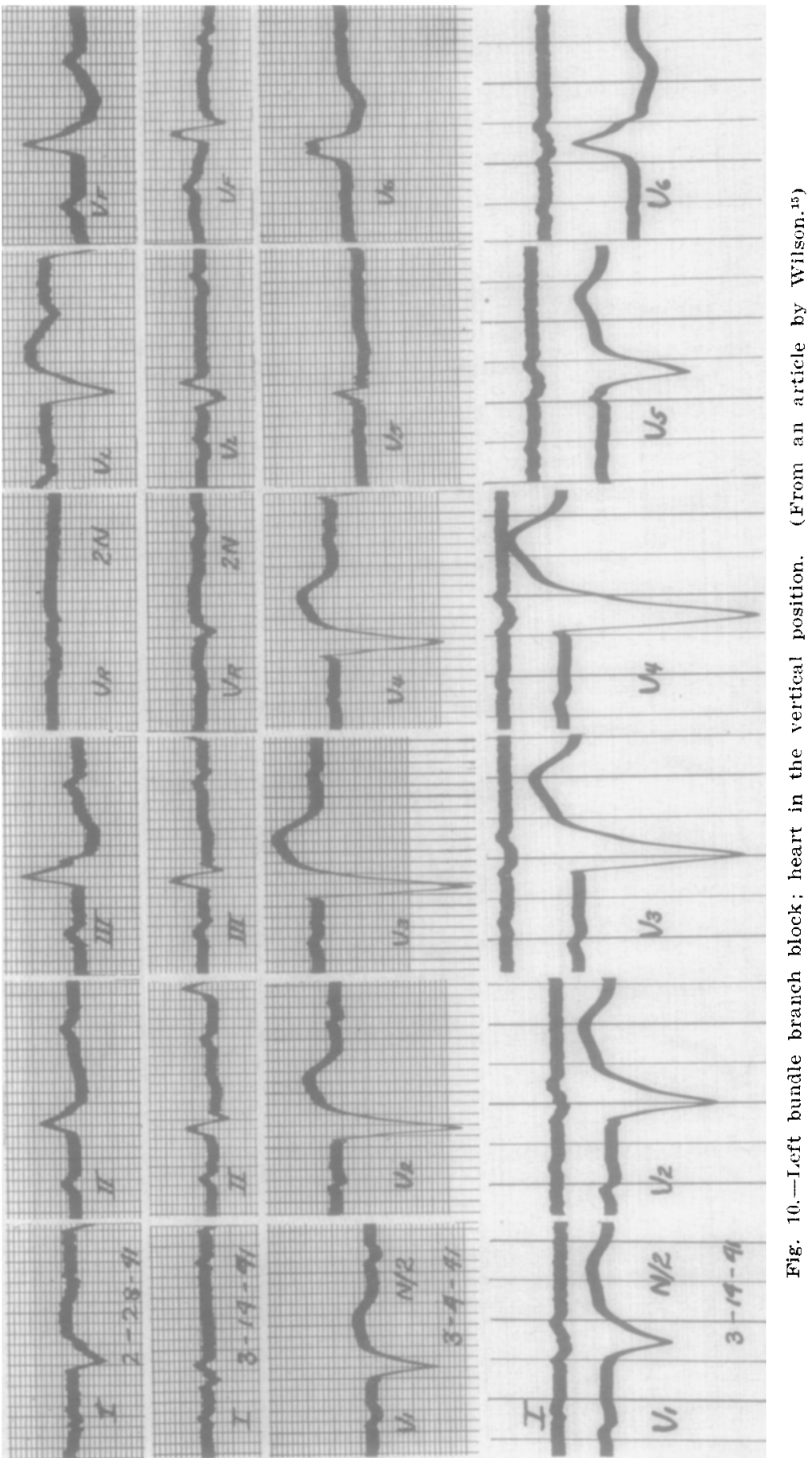


4 and on March 14, and the two' sets of curves differ only as regards the form of the ventricular complexes of Lead $V_{5}$. Differences of this kind have no important significance. They are apparently due to variations in the position of the heart or to slight variations in the locations of the precordial points explored. The precordial electrocardiograms demonstrate beyond question that the conduction defect was in the left branch of the His bundle and not in the right. ${ }^{i 5}$ When they are compared with the unipolar extremity curves taken on February 28, it is clear that the heart was then in the vertical position. On that date, the potential variations of the left arm were like those subsequently found on the right side of the precordium and the potential variations of the left leg like those found on the left side of the precordium. On March 14, the heart was in a somewhat different position. On that day, the potential variations of the left arm and of the left leg were apparently complicatcd mixtures of components transmitted from the surface of the right ventricle, and components derived from the surface of the left ventricle.

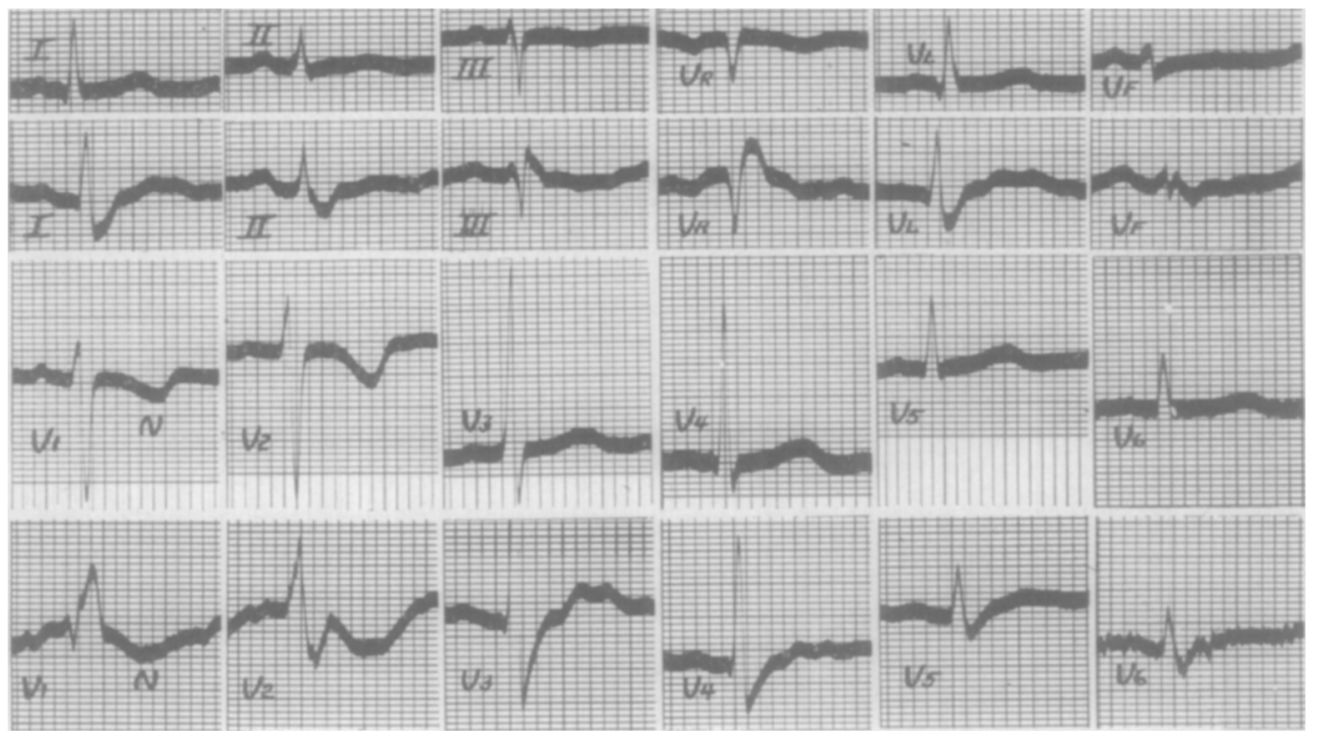

Fig. 11.--Right bundle branch block induced by quinidine; heart in the semihorizontal position.

The electrocardiograms reproduced in Fig. 11 are those of a young woman with ulcerative colitis who developed right bundle branch block on two different occasions after the administration of $0.4 \mathrm{Gm}$. of quinidine sulfate. Before the drug was given, slight left axis deviation was present, but, apart from inversion of the $\mathrm{T}$ waves in Lead $V_{2}$, both the standard and the precordial electrocardiogram were within normal limits. In both sets of curves, the ventricular complexes of Lead $V_{L}$ resemble those of Leads $V_{5}$ and $V_{6}$, and the deflections of Lead $V_{F}$ are 
small. The heart was then in the semihorizontal position. It will be noted that the onset of right branch block produced more striking changes in the form of the ventricular complexes of Lead $V_{1}$ than in the form of those of Leads $V_{5}$ and $V_{6}$. In the latter, the $R$ wave was slightly smaller and the $S$ deflection deeper and broader after the block than before; in the former, the deep $s$ originally present was replaced by a broad slurred $R^{\prime}$ when the block developed. When the cardiac mechanism was normal, the transitional zone which yields complexes intermediate in form between those obtained from points farther to the left and those obtained from points farther to the right lay between precordial points 2 and 3 . When right branch block was present, it lay between points 1 and 2 ; only the first precordial lead yielded ventrieular complexes of the kind seen in direct leads from the surface of the right ventricle. On the second occasion on which right branch block was induced by quinidine, this apparent shift in the transitional zone did not oecur.

The electrocardiograms reproduced in Fig. 12 represent right bundle branch block in a man whose heart was in the senivertical position. The precordial electrocardiogram displays the features usually associated with this conduction defect; the lransitional zone lay belween points 3 and 4 . In the standard limb leads the ventricular complexes are of the concordant type. The potential variations of the left leg were like those of the left side of the precordium and the potential variations of the left arm were very small.

The electrocardiograms reproduced in Fig. 13 are those of a man, aged 62 years, with arteriosclerotic heart discase. The ventricular complexes of the standard limb leads suggest that left branch block was present. It should be noted, howerer, that a $\mathrm{Q}$ and an $\mathrm{S}$ deflection are present in Lead I; both of these components are extremely rare in this lead in left branch block but common in right. The precordial electrocardiogram shows that the conduction defect was on the right side, but it is peculiar as regards the size of the $R$ deflection in Iseads $V_{5}$ and $V_{6}$. The small size of the $R$ waves in these leads suggests that the block was complicated by some factor which diminished the magnitude of the forces produced by activation of the anterolateral wall of the left ventricle. Although no unipolar limb leads were taken, the form of the complexes of the standard limb leads shows that the potential variations of the left leg were like those of the left side of the precordium and the potential variations of the left arm like those of the right side of the precordium. The heart was, therefore, in the vertical position.

When the QRS interval measures 0.12 second or more, and the QRS complex in Lead I is monophasic and consists of a broad, slurred, flattopped, or bifid $R$ deflection, the precordial curves are, almost always, characteristic of left branch block. When the QRS interval measures 0.12 second or more, and the QRS complex in Lead I is biphasic or triphasic and ends with a broad, sturred or notehed $\mathrm{S}$ deflection, the 
precordial curves are, in the vast majority of cases, characteristic of right bundle branch block. The oceasional exceptions to these general rules are due to a number of different causes. When the heart is in the vertical position, the limb leads may suggest that right branch block is

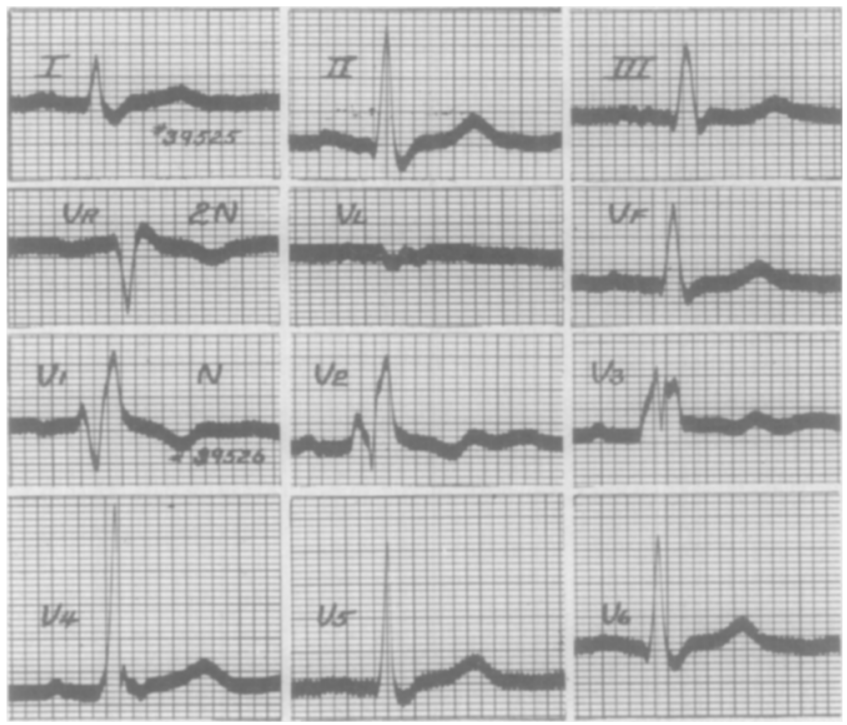

Fig. 12.-Right bundle branch block; heart in the semivertical position.

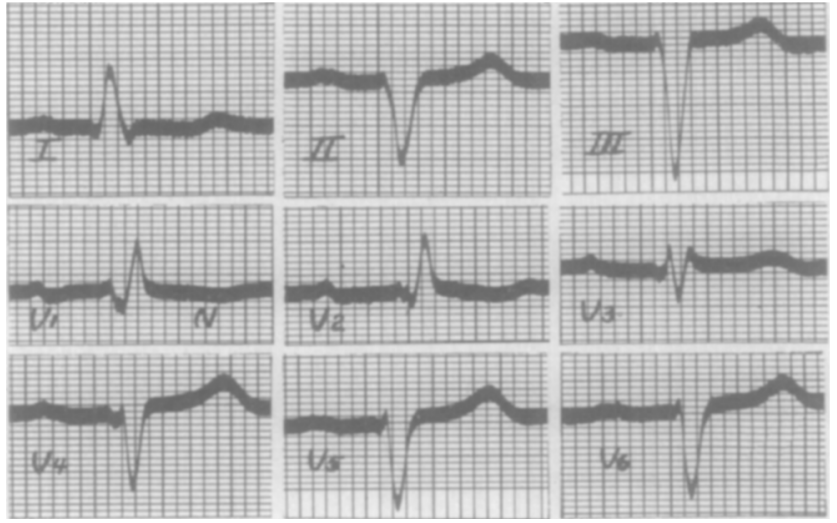

Fig. 13.-Right bundle branch block $\underset{\text { by }}{\text { heart in the vilson. }{ }^{.5} \text { ) }}$ - Rtical position. (From an article

present when the precordial curves are characteristic of left branch block, and vice versa. In other instances, the prolongation of the QRS interval is due to a general depression of the conductivity of the Purkinje tissue or to local lesions involving the subendocardial Purkinjc network over a considerable area ("arborization block"). ${ }^{16}$ The latter is seen most often in association with extensive myocardial infaretion.

When the QRS interval measures 0.12 second or more, and the precordial electrocardiogram shows some but not all of the features typical 
of right or of left branch block, the conduction defect cannot be located with certainty. Sometimes this situation arises because this defect is associated with lesions which modify its effects upon the electrocardingram. Sometimes it depends upon peculiarities in the extent or location of the transitional zone, which separates the parts of the precordium that display potential variations like those of the anterior surface of the right ventricle, from the parts of the precordium that display potential variations like those of the anterolateral surface of the left ventricle. This may be so far to the left or so far to the right that all of the six standard precordial leads yield ventricular complexes of the same type. When this happens, it is desirable to take additional unipolar leads so arranged as to cross this zone. It is still impossible to say why the transitional zone is so variable in width and location. Finally. it should be emphasized that in many cases of bundle branch block there is no obvious relation between the form of the ventricular complexes of the precordial leads and the form of the ventricular complexes of the limb leads. In these cases the position of the heart must be classed as indeterminate.

When the QRS interval is abnormally long but measures distinctly less than 0.12 second, the precordial curves are seldom characteristic of complete bundle branch block. In rare instances they are typical of right branch block even though the QRS interval of the limb leads is not much greater than 0.10 . In most of the cases of this kind that we have seen we suspected that the right ventricle was greatly hypertrophied, and it is possible that this condition was in part responsible for the form of the precordial electrocardiogram.

Unipolar precordial leads make it possible to diagnose incomplete right bundle branch block in many cases in which it cannot be recognized by other means. Except under special circumstances, the diagnosis of incomplete left branch block is still very difficult to make with certainty.

The electrocardiograms reproduced in Figs. 14 and 15 represent the alternation of complete with incomplete right branch block. The first ventricular complex of each pair represents complete, and the second incomplete, block. It will be noted that as regards their earliest components the paired complexes are identical in all leads. In the case of the limb leads and the leads from the left side of the precordium ( $\boldsymbol{V}_{5}$ and $V_{6}$ ), the sole difference lies in the width of the final QRS component and the size of the $\mathrm{T}$ deflection. In the leads from the right side of the precordium $\left(V_{1}\right.$ and $\left.V_{2}\right)$, from the tip of the ensiform process $\left(V_{E}\right)$, and from the right nipple line, the difference is much greater; the large final $R^{\prime}$ deflection of the first complex is very much smaller $\left(V_{1}\right.$ and $\left.V_{E}\right)$ or embryonic ${ }^{*}$ in the second $\left(V_{2}\right)$. The presence of such

*This term is used to indicate that the peak of this deflection does not rise above the level of the trace at the beginning of the QRS interval. 
small or embryonic $R^{\prime}$ deflections, in addition to an initial $R$ wave in the leads from the right side of the precordium, strongly suggests that incomplete right branch block is present, particularly when the QRS interval is slightly increased and there is a broad $S$ wave in Lead I and in the leads from the left side of the precordium. It is advisable to take precordial leads whenever the QRS complex of Lead I is of this kind.

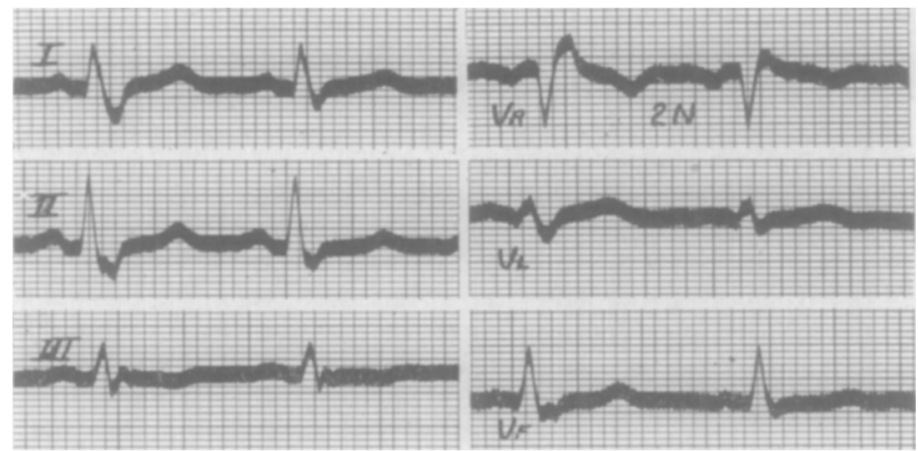

Fig. 14.-Complete and incomplete right bundle branch block; standard and unipolar limb leads.

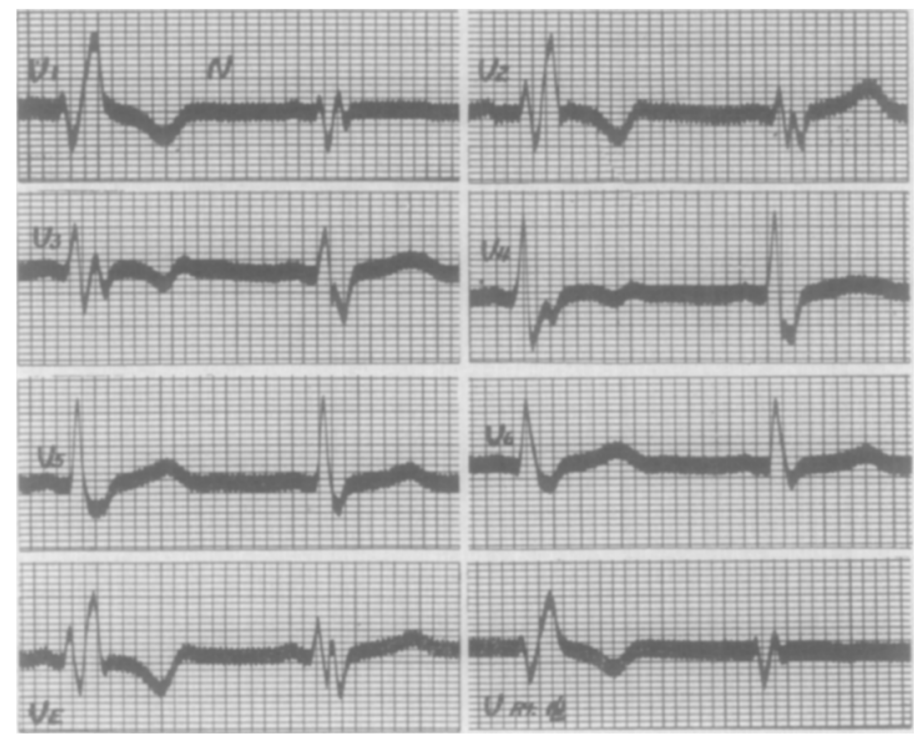

Fig. 15.-Complete and incomplete right branch block; precordial leads.

In incomplete left bundle branch block, the precordial electrocardiogram resembles that seen in preponderant hypertrophy of the left ventricle and the two conditions frequently coexist. The presence of a $Q$ deflection in the leads from the left side of the precordium excludes the former, but the absence of this deflection does not differentiate one from the other. 
Transient Branch Block.-In the vast majority of the cases of human bundle branch block, there is no way of knowing whether all the electrocardiographic abnormalities present are due to the conduction defect or whether some of them are the result of the other lesions which are almost always present. Cases in which the electrocardiogram is normal after branch block disappears, or immediately before it develops, are, therefore, of unusual interest. In such cases the effects produced by the block are uncomplicated by other factors.

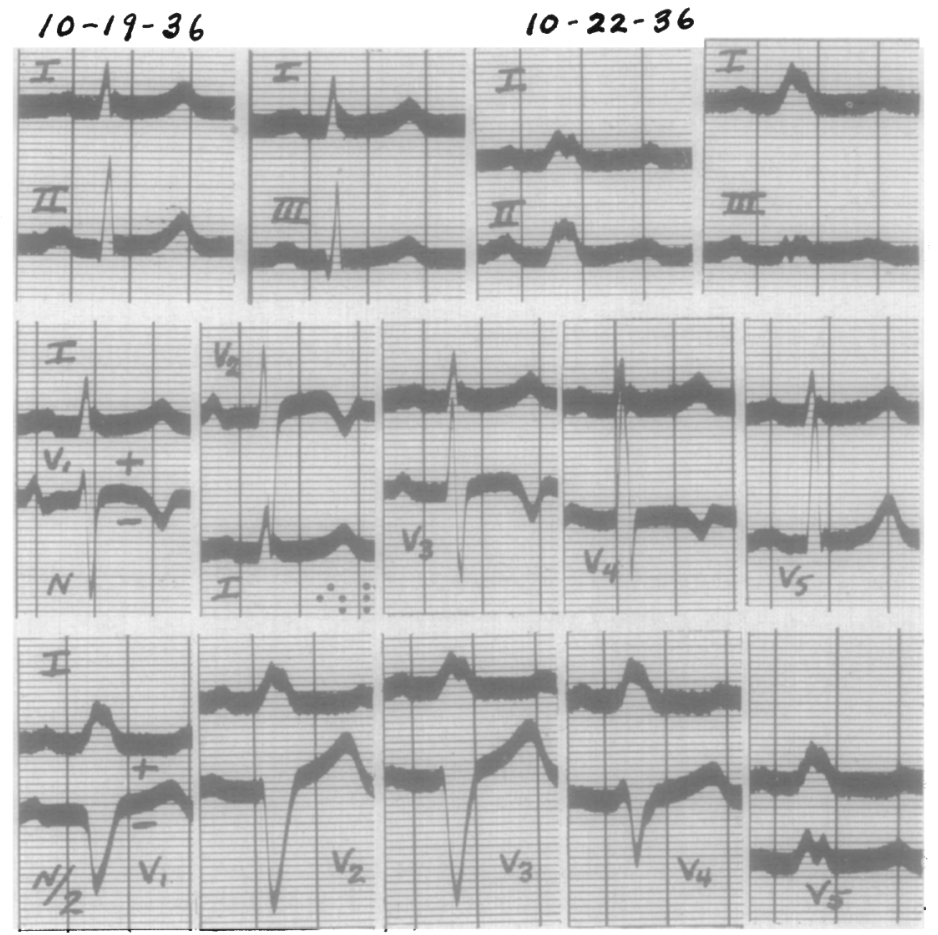

Fig. 16. - Transient left branch block.

The electrocardiograms reproduced in Fig. 16 are those of a woman, aged 55 years, who had symptoms suggesting hyperthyroidism. She was under observation from Oct. 13, 1936, until Nov. 3, 1936. Left branch block was present when the patient was seen for the first time, but, on October 19, the standard extremity curves were normal. At this time the precordial curves were normal as regards the QRS complexes, but the $\mathrm{T}$ waves were sharply inverted in Leads $V_{1}, V_{2}, V_{3}$, and $V_{4}$. This case conforms to the rule that abnormalities which are confined to the leads from the right side of the precordium are not accompanied by similar abnormalities in the limb leads. It illustrates the value of precordial leads in detecting lesions which produce electrocardiographic disturbances of this kind. On October 22, the branch block was again present. In the standard leads the broad notched QRS deflections were of 
low voltage and of the concordant type. In the precordial leads the voltage of the chief QRS deflection was so large that, in order to record it properly, it was necessary to reduce the string galvanometer to onehalf the normal sensitivity. It will be noted that the conduction defect greatly reduced the size of the $R$ waves in the leads from the right side of the precordium, abolished the inversion of the $T$ waves previously present in these leads, and shifted the transitional zone to the left. In the leads from the left side of the precordium $\left(V_{5}\right.$ and $\left.V_{6}\right)$, it gave rise to monophasic QRS complexes consisting of broad, bifid $\mathrm{R}$ deflections. It is evident that the heart was in the semivertical position.

The electrocardiograms reproduced in Fig. 17 are those of a woman, aged 24 years, with rheumatic heart disease. She was under observation from Nov. 16, 1938, until April 25, 1939. When she was first seen, the electrocardiogram displayed rather large $\mathrm{P}$ deflections, but was not definitely outside normal limits. On April 5, the P-R interval measured 0.32 second, and right branch block was present. On April 14, the electrocardiogram was normal and precordial leads were taken. On April 25, right branch block was again present and the precordial leads were repeated. It will be noted that the block gave rise to a large final $R^{\prime}$ deflection in Leads $V_{1}$ and $V_{2}$, inverted or flattened the $T$ waves in the first four precordial leads, made the $R$ deflections somewhat smaller in the leads from the left side of the precordium, and produced broad $\mathrm{S}$ waves in Leads $V_{5}$ and $V_{6}$.

Ventricular Hypertrophy.-Preponderant hypertrophy of the left ventricle increases the thickness and the bulk of its walls. When uncomplicated, its chief effect is, therefore, to exaggerate the normal difference in this respect between this chamber and its fellow. It is not surprising, then, that in left ventricular hypertrophy, unipolar precordial leads yield curves which, in their broader aspects, are not unlike those obtained when the heart is normal. The chief differences are these: In left ventricular hypertrophy the voltage of the chief deflection of the QRS group is on the average much greater than normal, and the QRS interval is increased to 0.10 or even 0.11 second. In the leads from the right side of the precordium the $\mathrm{R}$ deflections are, on the average, much smaller than normal and may be absent. ${ }^{17}$ The transitional zone is, as a rule, much displaced to the left. In the leads from the left side of the precordium, $R$, and often $Q$ as well, is $a b$ normally large; the peak of $R$ oceurs abnormally late in the QRS interval; and the $\mathrm{T}$ deflections are inverted.

By increasing the bulk and thickness of its walls, preponderant hypertrophy of the right ventricle tends to abolish or reverse the normal difference between it and the left. In high-grade right ventricular hypertrophy, the precordial curves are, as regards their broader aspects, somewhat like those obtained in right branch block, but the QRS inter- 
val is not increased and the QRS deflections are not ordinarily loroad, slurred, or notehed. On the average, the voltage ot the chict QRS defiection is above normal although not as large as in lett ventricular hypertrophy. In the leads from the right side of the procordium,

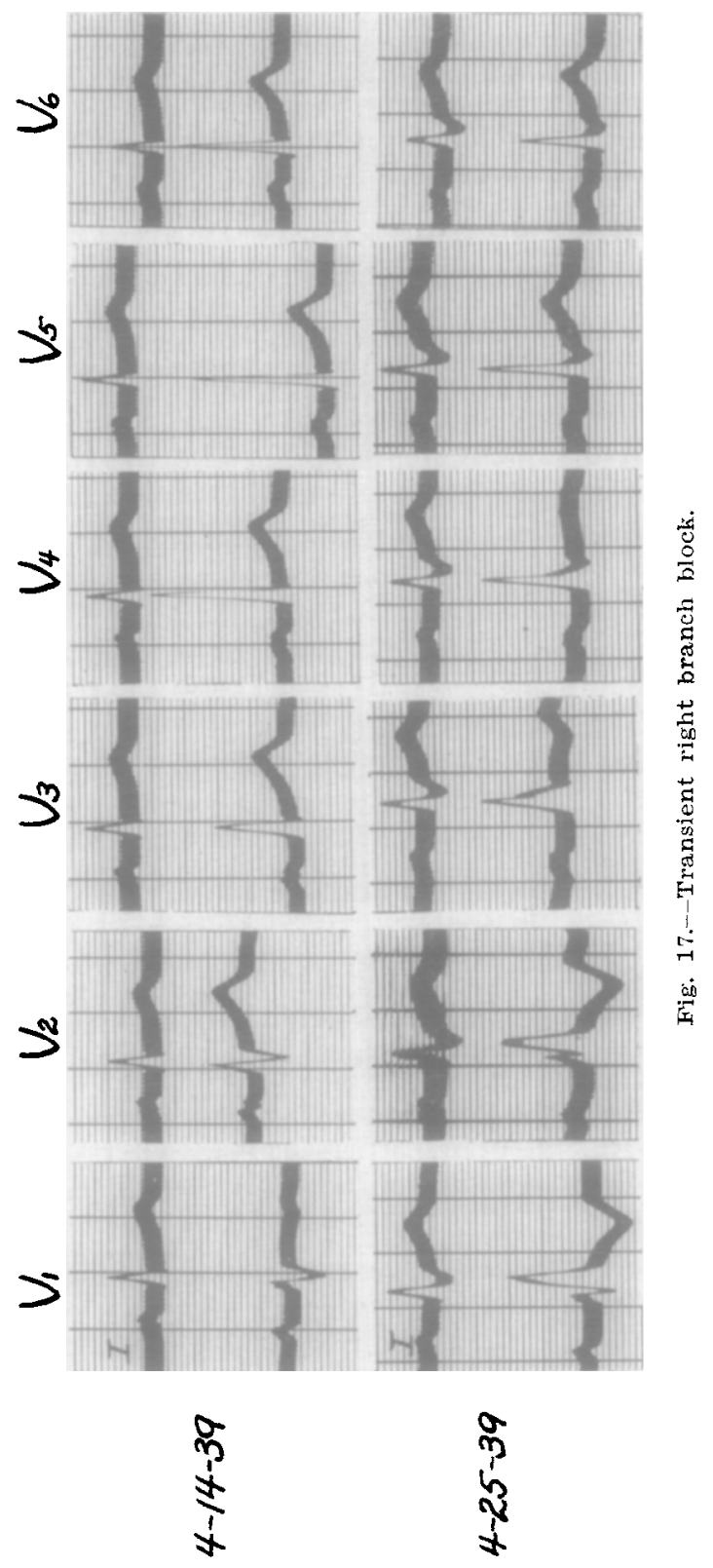

the $R$ wave is very large, $Q$ is frequently present, $S$ is usually absent, and the $\mathrm{T}$ deflections are commonly inverted. In some instances a small initial upward deflection precedes the first downward deflection of the 
QRS group, but we suspect that the presence of such a summit may be due to incomplete right branch block. In the leads from the left side of the precordium the $R$ deflections are abnormally small and the $S$ deflections abnormally large. In other words, the precordial curves are opposite in type to those obtained when the heart is normal.

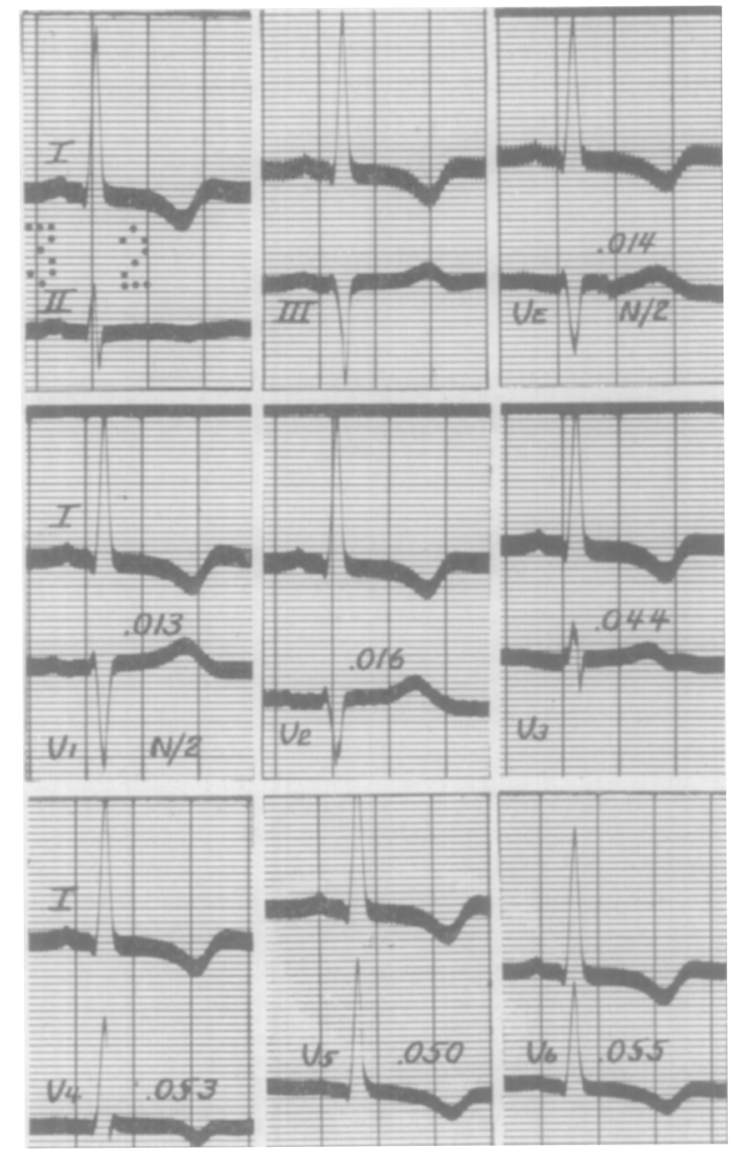

Fig. 18.-Left ventricular hypertrophy; heart in the horizontal or semihorizontal position.

In left ventricular hypertrophy the position of the heart has the same effect upon the mean electrical axis of QRS as in normal subjects. The electrocardiograms reproduced in Fig. 18 are those of a man, aged 54 years, with hypertensive heart disease. The standard extremity curves display left axis deviation, a QRS interval measuring approximately 0.10 second, and prominent $Q$ waves, abnormally large $R$ deflections, and inverted $\mathrm{T}$ waves in Lead $\mathrm{I}$. The precordial curves, which were taken with the galvanometer at one-half the normal sensitivity, are characteristic of left ventricular hypertrophy. Although no unipolar limb leads were taken, it is clear that the heart was in the horizontal or semihorizontal position. 
The electrocardiograms reproduced in Fig. 19 are those of a woman, aged 50 years, with hypertensive heart disease. No axis deviation is present, but the $R$ deflections are ahmormally tall and the $T$ waves inverted in all of the standard limb leads. The QRS interval is slightly increased. The precordial curves, which were taken with the galvanometer at one-half the normal sensitivity, are characteristic of left ventricular hypertrophy. The potential variations of the left leg were like those of the left side of the precordiun and the potential variations of the left arm were small. The heart was in the semivertical position.

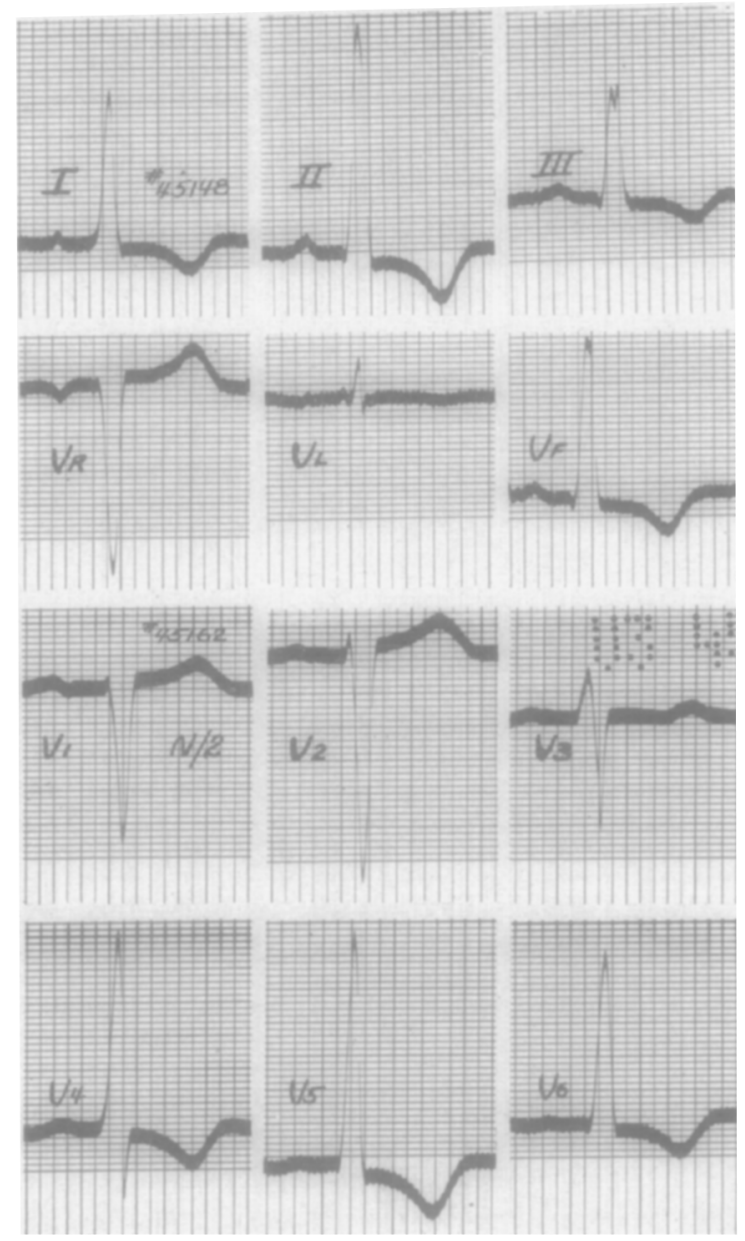

Fig. 19.-Left ventricular hypertrophy; heart in the semivertical position.

The electrocardiograms reproduced in Fig. 20 are those of a young man, aged 16 years, with rheumatic heart disease, aortic stenosis and regurgitation and probably mitral stenosis, and an enormously enlarged heart. Right axis deviation is present, the $\mathrm{T}$ deflections are inverted in Leads II and III, the P-R interval is increased, and the P deflections 
are large and deformed. Seven precordial leads were taken, the last, marked $V_{7}$, from the posterior axillary line at the level of the apex, which was not far inside this point. The precordial curves, which were taken with the galvanometer at one-half the normal sensitivity, are characteristic of left ventricular hypertrophy. Although unipolar limb leads were not taken, it is evident the potential variations of the left leg were like those of the left side of the precordium and the potential variations of the left arm like those on the right side of the precordium. The heart was, therefore, in the vertical position.

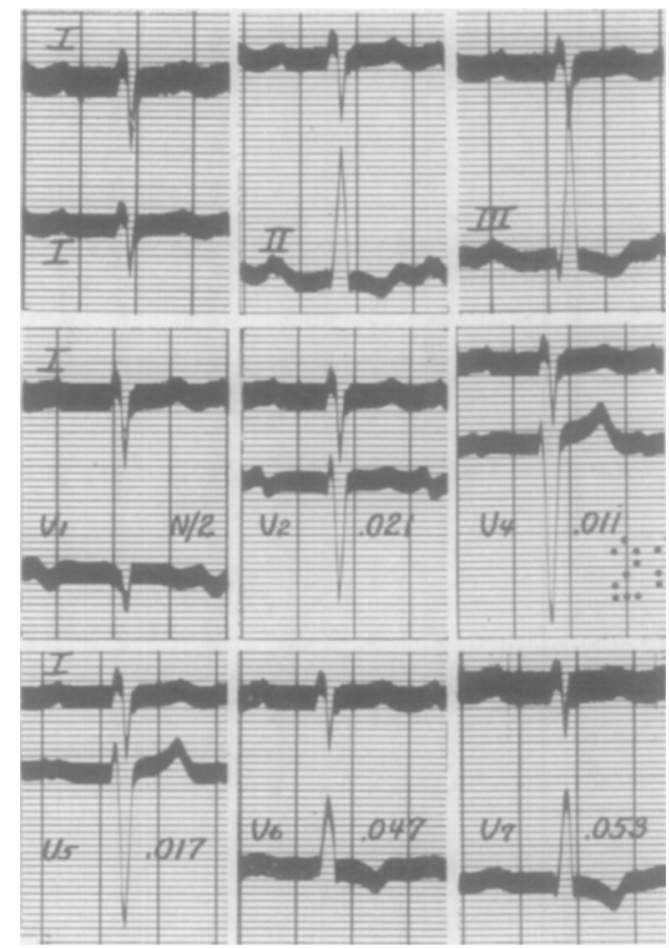

Fig. 20.-Left ventricular hypertrophy; heart in the vertical position. (From an article by Wilson, Johnston, Cotrim, and Rosenbaum. ${ }^{13}$ )

The electrocardiograms of two patients with right ventricular hypertrophy are reproduced in Fig. 21. The eurves of the upper set are those of a woman, aged 34 years, who had pulmonary hypertension of unknown origin. The limb leads display conspicuous right axis deviation. The precordial curves are characteristic of right ventricular hypertrophy; tall $R$ waves, prominent $Q$ waves, and inverted $T$ waves are present in Lead $V_{1}$, and small $\mathrm{R}$ and deep $\mathrm{S}$ waves in Leads $V_{5}$ and $V_{6}$. The potential variations of the left arm were like the potential variations of the left side of the precordium, and the potential variations of the left leg resembled those of the right side. The heart was in the horizontal position. The curves of the second set are those of a 
man, aged 40 years, with mitral stenosis and aortic insufficiency. The precordial curves are characteristic of right ventricular hypertrophy. Large $\mathbf{S}$ waves and small $R$ waves are present in all of the standard

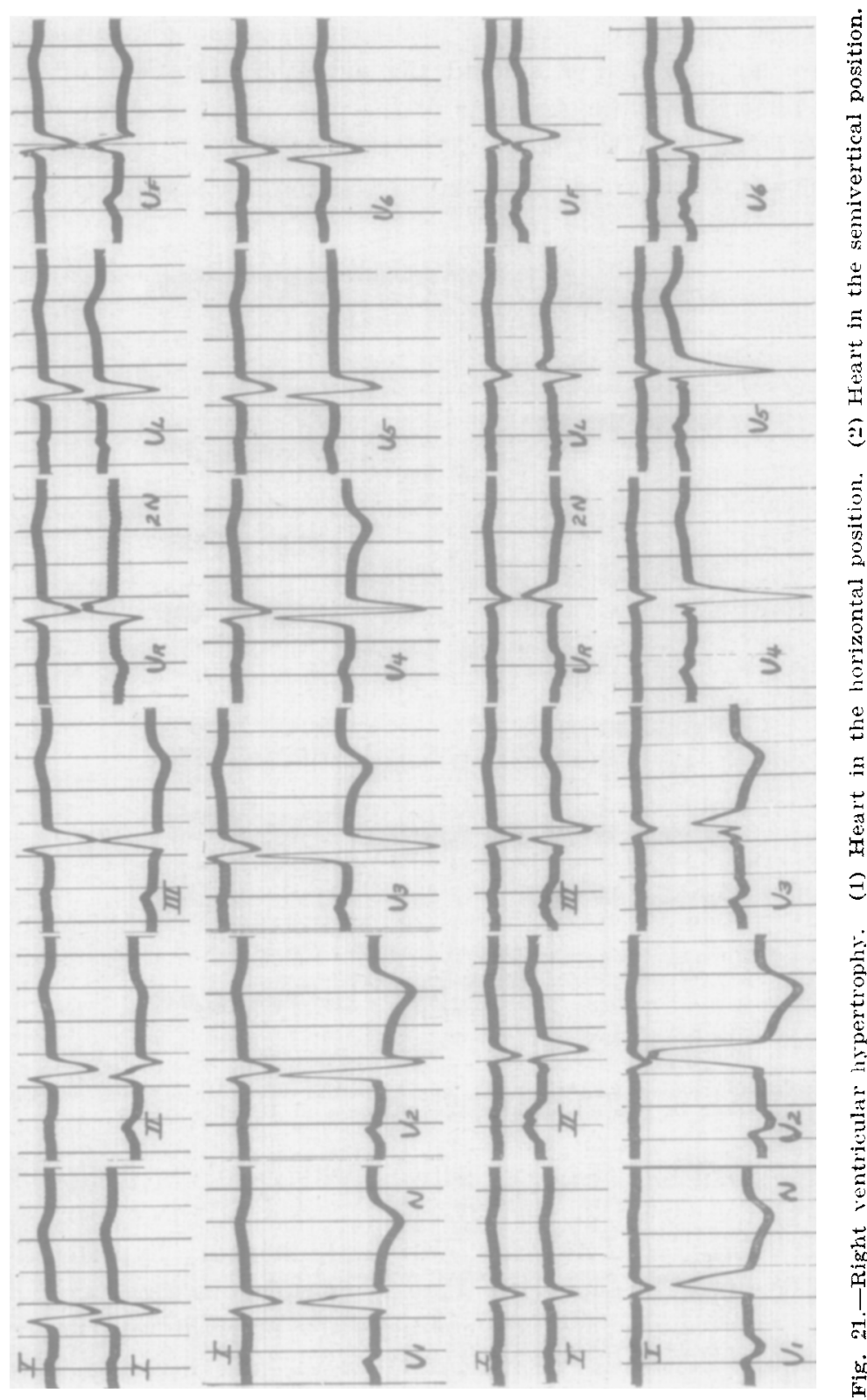

limb leads. Here the ventricular complexes of the unipolar leg lead are like those of Leads $V_{5}$ and $V_{6}$; the complexes of the right arm lead are like those of Lead $V_{1}$, and the complexes of the left arm lead apparently represent a mixture of the potential variations of the other two ex- 
tremities. In this case the heart was apparently in the semivertical position.

When the heart is greatly enlarged, it is ordinarily in the horizontal or semihorizontal position. In either ease, left ventricular hypertrophy produces left, and right ventricular hypertrophy produces right, axis deviation. When the heart is in the semivertical position, left ventricular hypertrophy produces abnormally tall $\mathbf{R}$ waves and, usually, inverted $\mathrm{T}$ waves, and right ventricular hypertrophy produces large $\mathbf{S}$ waves in all of the standard limb leads. When the heart is in the vertical position, left ventricular hypertrophy produces right, and right ventricular hypertrophy should produce left, axis deviation, although we have not as yet seen the latter occur.

The precordial curves of left ventricular hypertrophy are distinguished from those of left bundle branch block by the absence of slurred or bifid $R$ waves and the great frequency of $Q$ deflections in the leads from the left side of the precordium. In left branch block, the presence of $Q$ in these leads suggests that the diagnosis is wrong or that septal lesions which prevent initial positivity of the left ventrieular cavity are present. The QRS interval is almost always less than 0.12 second in hypertrophy, whereas, in block, it equals or exceeds this figure.

The precordial curves of right ventricular hypertrophy are distinguished from those of right bundle branch block by the absence of double or bifid $\mathrm{R}$ waves in the leads from the right side of the precordium, by the small size of the $R$ waves in the leads from the left side of the precordium, and by the absence of a greatly increased QRS interval.

It must be admitted, however, that cases are frequently encountered in which it is impossible to say with certainty whether the electrocardiographic changes are due to hypertrophy alone or to hypertrophy plus incomplete bundle branch block.

Myocardial Infarction.-The character of the potential variations at the epicardial surface of an infaret depends upon the stage of infaretion and upon whether the whole thickness of the ventricular wall or only the subendocardial layers of muscle are involved. Immediately after ligation of the anterior descending coronary artery or one of its branches in experiments upon the dog's heart, direct leads from the region supplied by the vessel occluded display pronounced upward displacement of the RS-T junction, which persists for many hours but disappears when the muscle affected dies or recovers. ${ }^{18}$ This RS-T displacement is accompanied by a corresponding reduction in the voltage of the intrinsic deflection. When the whole thickness of the ventricular wall is involved and the infarcted muscle is already dead or ineapable of responding to the excitatory impulse, the potential variations of the part of the ventricular eavily adjacent to the infaret are faithfully transmitted to its epicardial surface. Since the ventricular cavities 
are normally negative throughout the QRS interval, direct leads from the central part of the infaret then yield QRS complexes which consist of a monophasie downward deflection (QS deflection). In many cases, some fraction of the muscle in this region remains alive and gives rise to an embryonic $R$ wave which produces a notch on the descending or ascending limb of the single QRS component. When the subendocardial layers of muscle are for the most part dead or incapable of responding to the cardiac impulse, and the outer layers of muscle are relatively normal, the embryonic $R$ rises above the base line and becomes a true $\mathrm{R}$ deflection. The QRS complex then consists of an $\mathrm{ab}$ normally large $Q$ followed by a small $R$ and often by an $S$ deflection also. Since infarets of the left ventricular wall are usually more extensive on the endocardial than on the epicardial side, QRS complexes of this kind are commonly seen in direct leads from the marginal parts of the infarcted area. ${ }^{19}$ These same zones also yield deep, sharply inverted $\mathrm{T}$ deflections due to an increase in the duration of the excited state at the epicardial surface. In the dog, the T-wave changes develop some hours after infaretion begins, and ordinarily disappear almost completely within twenty-four hours or less. In man, they commonly last for some wecks or months.

The potential variations of the epicardial surface of the infareted region are transmitted to the adjacent parts of the body. When the anterior wall of the left ventricle is involved, they are transmitted to the precordium; if the anterolateral wall is involved, they are also transmitted to the left arm. If the posterior wall is infarcted, they are usually transmitted to the ventricular levels of the esophagus, to the back, and to the left leg. The potential variations of points at some distance from the heart but on the same side of it as the infareted region are, of course, a mixture of components derived from the epicardial surface of the infarct, from regions adjacent to it, and from other parts of the ventricular surface. It is only when the infarct is large that characteristic curves are obtained by leading from regions distant from it. The potential variations of parts of the body on the side of the heart opposite the one involved, as regards their general character, are the inverse of those that occur at the surface of the infarct.

The diagnosis of myocardial infaretion can be made with certainty from the electrocardiogram alone only when characteristic changes in the QRS complex occur in association with RS-T displacement or typical changes in the $\mathrm{T}$ deflections. Changes in the QRS complex have more diagnostic value than displacement or changes in the $T$ waves when only electrocardiographic data are available, but they are not conclusive. When clinical data are available and are strongly suggestive of infarction, and serial curves are taken, the opposite is true because of the relatively rapid, progressive changes which the $\mathbf{T}$ complex undergoes. Similar changes may occur in pericarditis, but usually this ean be ruled out on clinical grounds. 
From the electrocardiographic standpoint, there are many types of infarcts which differ one from another as regards the character of the electrocardiographic changes and the leads in which they occur. Whether some of these varieties are determined by the position of the heart rather than the location and extent of the infarcted region, only the future can decide.

T.Wave Changes Only.-The electrocardiograms reproduced in Fig. 22 are those of a physician, aged 67 years, who was first seen on Aug. 7, 1934. At that time, the standard limb leads showed a considerable shift of the electrical axis to the left, but no other notable features. Myocardial infarction occurred early in March, 1940. Six weeks later (April 25, 1940) there was sharp inversion of the $\mathrm{T}$ deflections in all of the precordial curves although the limb leads showed no significant changes. After eight months, all electrocardiographic evidence of infarction had completely disappeared. The patient made an uneventful recovery. We have seen no instance of serious cardiac impairment following anterior infarction which produced no electrocardiographic abnormalities other than sharp inversion of the $\mathrm{T}$ waves.

Transient QRS Changes.-The electrocardiograms reproduced in Fig. 23 are those of a man who began to have symptoms typical of coronary thrombosis at 6:40 P.M., on Oct. 6, 1936, while he was under treatment for prostatic hypertrophy. On the following day, the standard electrocardiogram showed extremely small QRS deflections and flat $\mathrm{T}$ waves in Lead I. In the precordial leads there was a progressive diminution in the size of $\mathbf{R}$ as the exploring electrode was moved from the first to the fourth position, and this deflection was abnormally small in I eads $V_{5}$ and $V_{6}$. A month later, the QRS deflections were normal, but there was sharp inversion of the $\mathrm{T}$ waves in all of the limb leads and in the last four precordial leads. The patient's recovery was complete and uninterrupted.

It is apparent that, in this instance, the infareted muscle was, for a time, incapable of responding to the excitatory impulse, but it was not dead and subsequently recovered its excitability. Very pronounced alterations in the QRS complex of the kind observed in this case seldom disappear completely, but they often undergo a very slow and much less pronounced retrogression. Very often, elear-cut and characteristic deformities of this complex and, much more rarely, residual changes in the $\mathrm{T}$ waves are still present years after the cardiac accident. An instance of this kind is illustrated in Fig. 24. The patient developed myocardial infarction in May, 1934, when he was 23 years old. The electrocardiograms taken at that time are not very different from those reproduced, which were made seven years later. When last seen, this young man was working and was free of symptoms. Note that the ventricular complexes of the unipolar left arm lead are like thosc of Lead $V_{5}$. 
Persistent RS-T Displacement.-The electrocardiograms reproduced in Fig. 25 are those of a man, aged 73 years, who began to have anginal pain late in May, 1941, and developed myocardial infarction on June 11 of that year. Very pronounced RS-T displacement of the kind commonly seen in very recent anterior infarction was still present on Nov. 7,

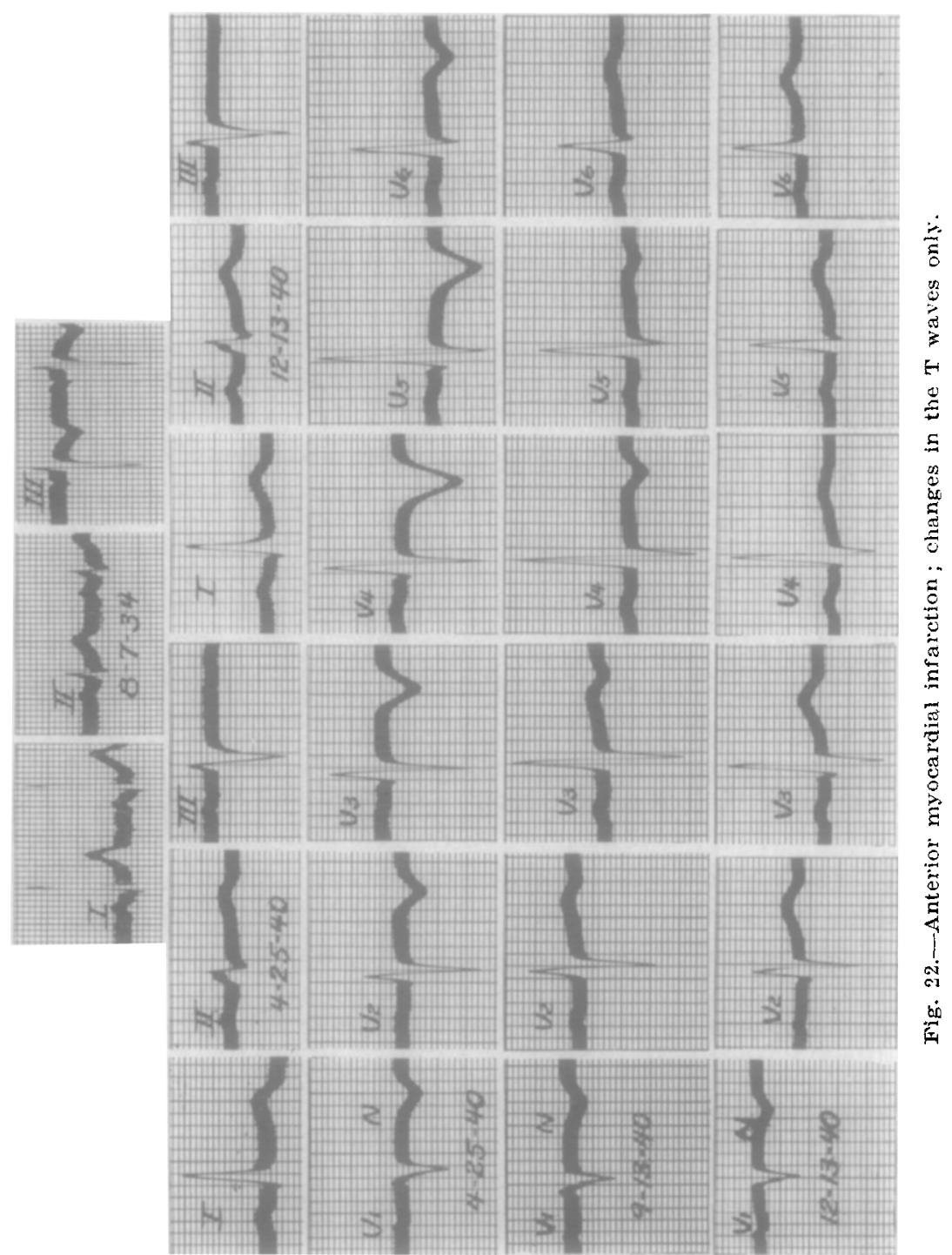

1941, when the first electrocardiogram was taken, and remained essentially unchanged on April 20, 1942. The patient developed congestive cardiac failure but had chest pain only during the earliest stage of his illness. Complete healing of the infareted muscle must have taken place long before the last curves were made. Ordinarily, pronounced RS-T displacement of the kind in question persists for a few 


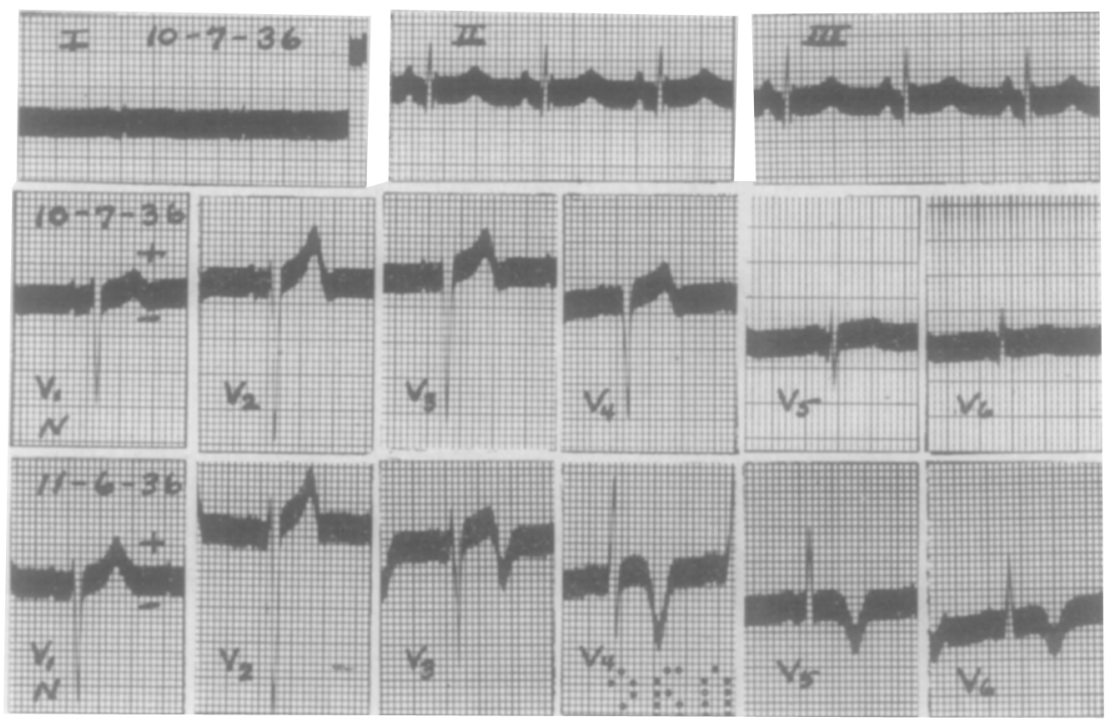

Fig. 23.-Anterior myocardial infarction; transient QRS changes.

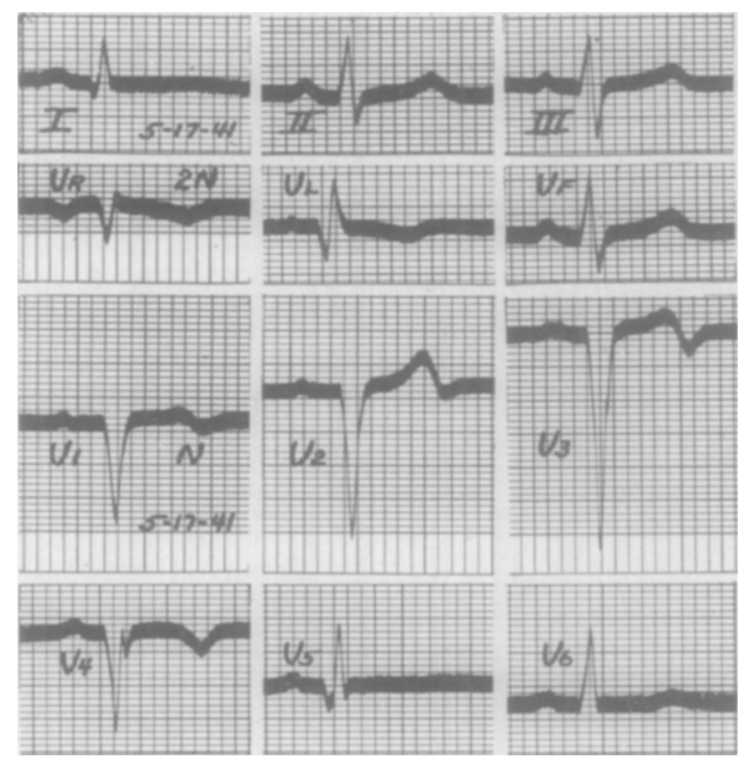

Coronary occlusion 7 years ago.

Fig. 24.-Anterior myocardial infarction; persistent changes in the QRS complex and residual changes in the $T$ waves. 
hours, or, at most, for a few days. Why it persists in rare inslancess for weeks or months is still a mystery. In three cases of this sort, one of which was observed by Langendorf, ${ }^{\circ}$ a ventricular aneurysm was present, but it is possible that the association was due to chance. There

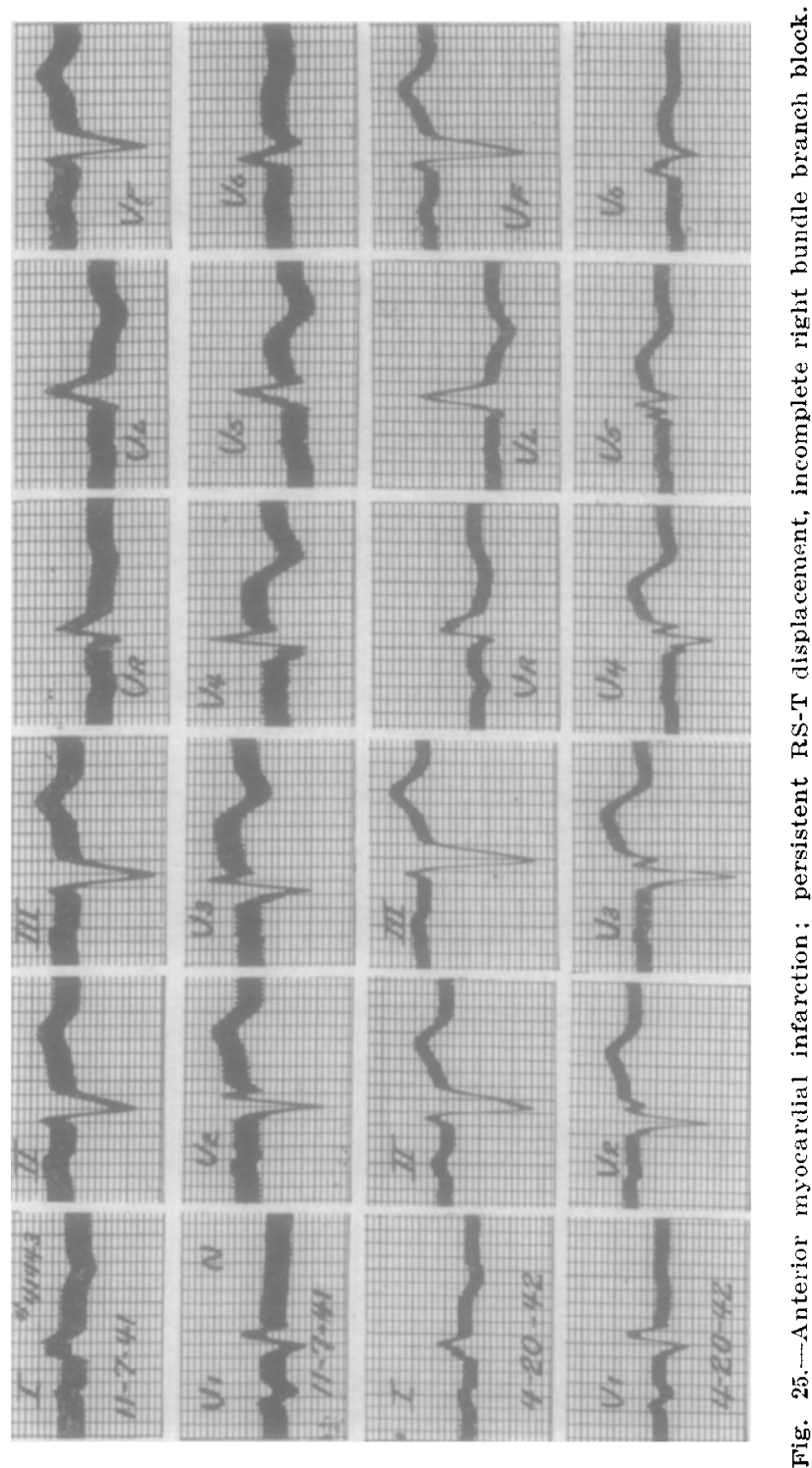

is no known reason why ventricular aneurysm should displace the RS.T junction or deform the RS-T segment in this way. In addition to RS-T displacement, both electrocardiograms display a QRS interval between 0.10 and 0.11 second in length, and late $R$ waves in the leads from the right side of the precordium. These findings strongly suggest that incomplete right branch block was present. 
Extensive Anterior Infarction.--In the three cases illustrated in Fig. 26, characteristic signs of infaretion were present in all of the six precordial leads, or in all except the first. In such instances it must be assumed that the region involved is a very large one. The first patient was a woman, aged 48 years, who began to have symptoms typical of coronary thrombosis at 9:45 P.M. on Aug. 9, 1938. On the

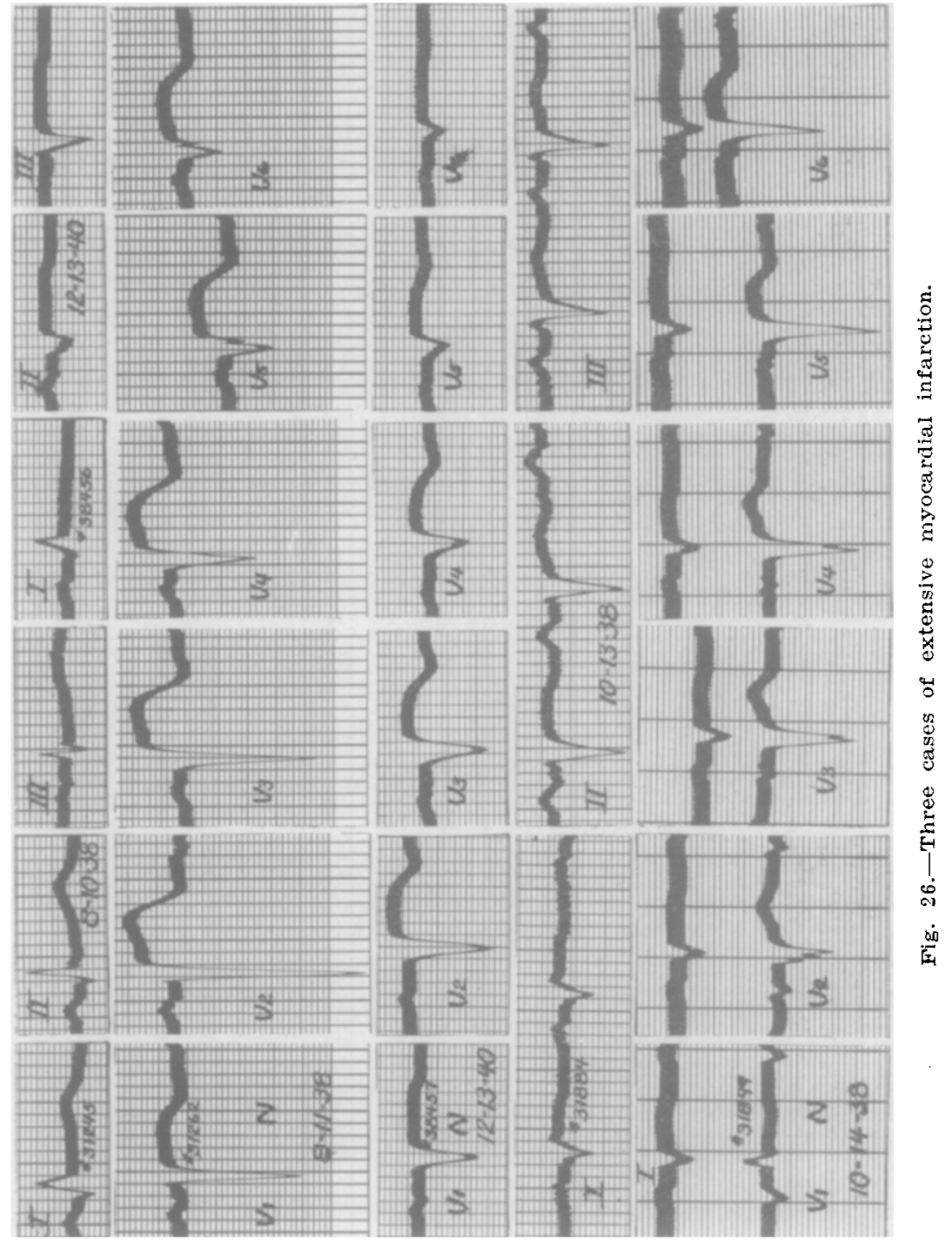

next day, small QRS deflections, prominent $\mathrm{Q}$ waves, and upward displacement of the RS-T junction were present in Lead I. On Angust 11, the precordial electrocardiogram displayed large QS deflections and conspicuous upward RS-T displacement in all leads except $V_{1}$. The second patient was a man, aged 71 years, who began to have severe 
pain in the chest at 9:45 r.M., on Dec. 10, 1940. On December 13, the changes in the precordial leads were almost identical with those that occurred in the case just described. Both patients recovered.

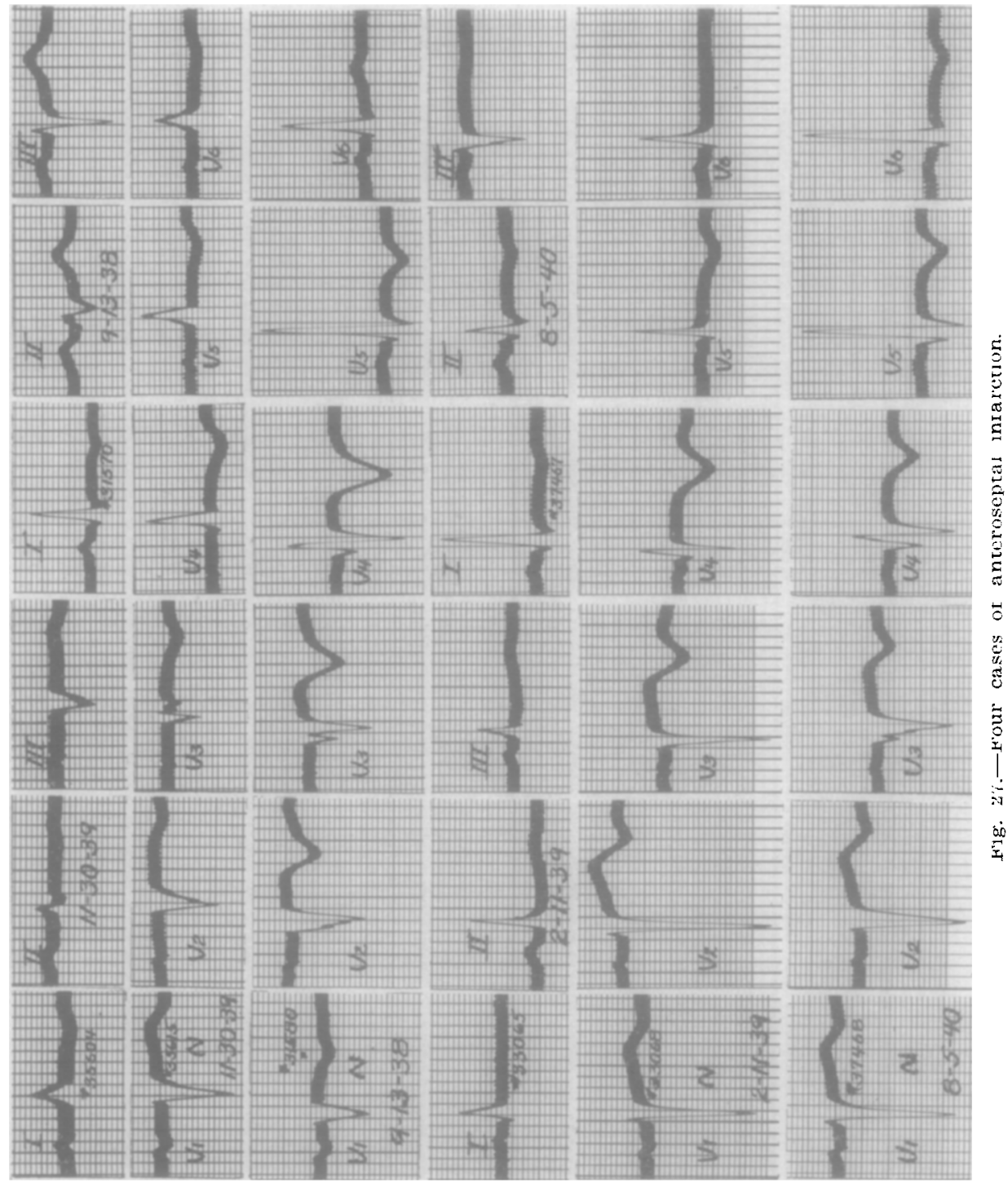

The third patient was a man, aged 48 years, who had severe attacks of anginal pain on Feb. 1, and on May 30, 1938. The first electrocardiogram was taken on Oct. 14, 1938. At that time there were very large $Q$ deflections in Lead I and large $Q S$ deflections in the last fivo precordial leads. Probably because the infaret was an old one, no pronounced RS-T displacement or typical $\mathrm{T}$-wave changes were present. The patient died on Oct. 16, 1938, and autopsy diselosed an infarct 
measuring 9 by $10 \mathrm{~cm}$., which involved the lower two-thirds of the anterolateral, and the lower one-third of the posterior, wall of the left ventricle. The cavity of this chamber was almost completely filled by a thrombus.

Anteroseptal Infarction.-In the four cases illustrated in Fig. 27, the diagnostic signs of infarction (characteristic QRS combined with characteristic RS-T or T-wave abnormalities) were confined to the leads from the right side of the precordium. Leads $V_{5}$ and $V_{6}$ display alterations in the $\mathrm{T}$ waves which are more or less typical but no unequivocal changes in the QRS complexes. Since the potential variations of the left arm are like those of the left side of the precordium in the majority of the cases of anterior myocardial infarction, the changes in the ventricular complexes of Ieat I are as a rule no greater than those displayed by the ventriculal complexes of lead $V_{5}$. An electrocardiographic diagnosis of anterior infarction, therefore, cannot be made with certainty in cases of the kind under consideration unless leads from the right side of the precordium are employed. Since the potential variations of the infarcted region are referred to the right side of the precordium, we may assume that the anteroseptal wall of the left ventricle is involved.

The first patient was a man, aged 68 years, who developed myocardial infarction in September, 1939. The electrocardiogram, taken on November 30 of that year, shows notched QS deflections in Leads $V_{2}$ and $V_{3}$ and inversion of the $\mathrm{T}$ waves, not of the kind characteristic of infaretion, in Leads I, $V_{3}, V_{4}, V_{5}$, and $V_{i j}$. The rentricular complexes of Lead I closely resemble those of Lead $V_{i ;}$.

The second patient was a man, aged 57 years, who had a coronary accident about Sept. 1, 1938. The electrocardiograms, made on September 13, show characteristic changes in the T waves of Leads $\mathrm{I}, V_{2}, V_{3}$, $V_{4}$, and $V_{5}$. Characteristic changes in the QRS complexes are confined to Leads $V_{2}$ and $V_{3}$. The rentricular complexes of I iead $I$ are like those of Lead $V_{5}$.

The third patient was a woman, aged 48 years, who began to have symptoms typical of coronary thrombosis on Feb. 9, 1939. The electrocardiograms, taken on February 11, show signs diagnostic of infaretion in Lead $V_{3}$ and typical T-wave changes in Lead $V_{4}$. The ventricular complexes of Lead I are like those of Lead $V_{6}$.

The fourth patient was a man, aged 59 years, who had a coronary occlusion on May 14, 1940. The electrocardiograms of Aug. 5, 1940, display signs diagnostic of infarction in Leads $T_{3}$ and $V_{4}$ and characteristic T-wave changes in Leads $V_{5}$ and $V_{6}$. The ventricular complexes of Lead $I$ are like those of Luead $V_{i j}$.

In normal subjects the $R$ deflection steadily increases in height as the precordial electrode is moved from the first to the fourth position and 
then decreases as it is moved farther to the left. The decrease in the height of $\mathrm{R}$, and its eventual disappearance as the exploring electrode was moved across the right side of the precordium in a number of the cases just described, is a far more reliable sign of infarction than complete absence of this deflection in the first two or three precordial leads.

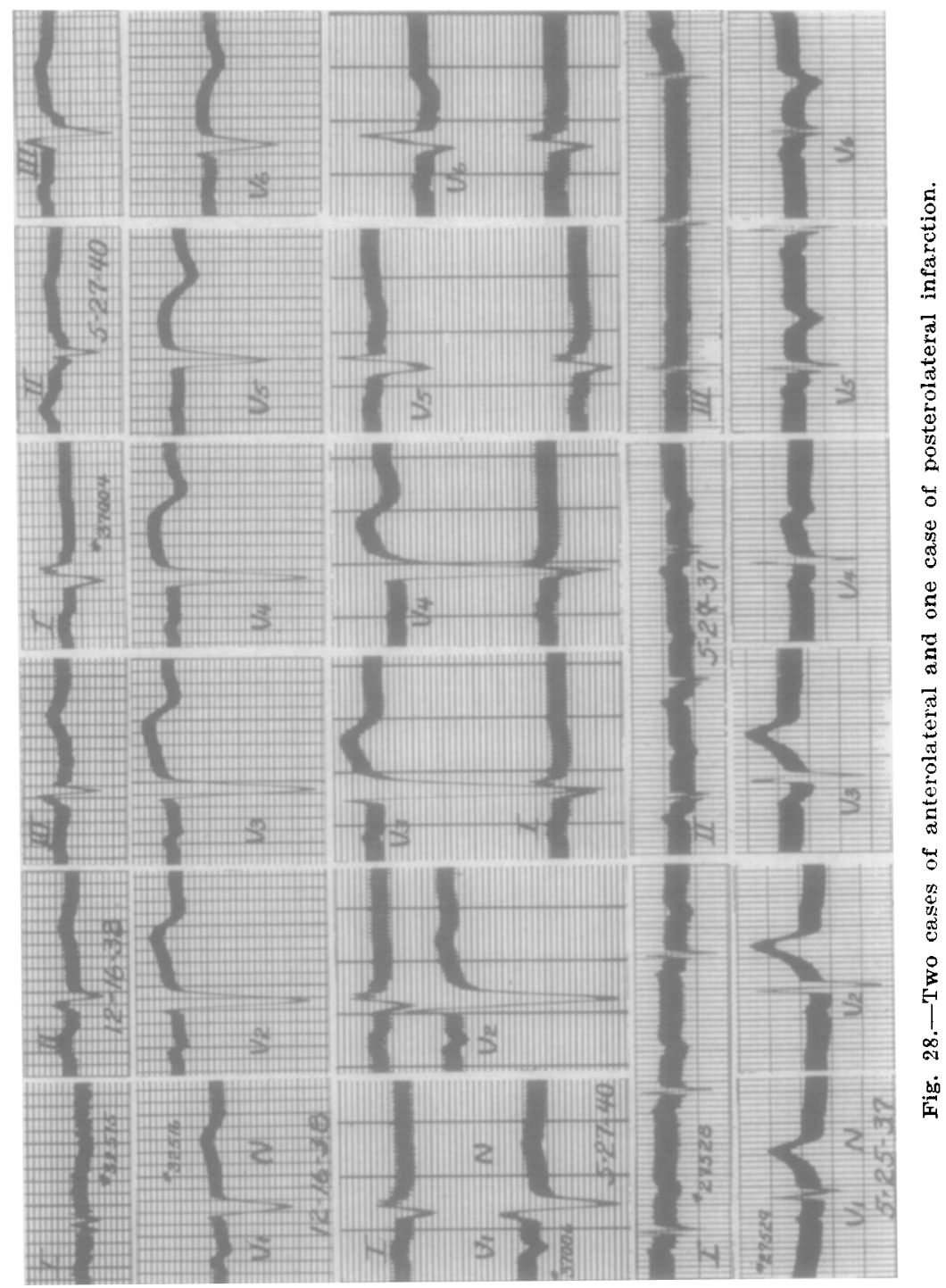

Anterolateral Infarction.--In the first two of the three cases illustrated in Fig. 28, the diagnostic signs of infarction in the chest leads are confined to the leads from the left side of the precordium, and it may be assumed that the anterolateral wall of the left ventricle was involved. 
The first patient was a man, aged 70 years, who had a severe attack of anginal pain on July 6, 1938. The precordial electrocardiogram, taken on December 16 of the same year, displays signs diagnostic of infarction in Leads $V_{4}$ and $V_{5}$, and abnormally small $R$ deflections associated with changes in the T complex in Lead $V_{6}$. In Lead $I$ the QRS deflections are extremely small, and suggestive changes in the $T$ waves are present.

The second patient was a man, aged 47 years, who was disoriented when admitted to the hospital, so that no history of his illness could be obtained. It was learned later that he had had anginal pain, but the time at which coronary thrombosis occurred could not be determined. The electrocardiogram displays deep $Q$ deflections in Lead I and in Lead $V_{5}$, and the ventricular complexes of these leads are very much alike. There is also a rapid decrease in the size of the $R$ deflection between Lead $V_{2}$ and Lead $V_{5}$. No characteristic modifications of the RS-T segment or $\mathrm{T}$ wave are present, probably because the infaret was an old one.

In infarcts of this kind, as a rule, there are changes diagnostic of infarction in the ventricular complexes of Lead $I$. Since the infaret involves the left upper margin of the heart, the potential variations at its epicardial surface are transmitted to the left arm.

The third case illustrated in Fig. 28 belongs to a different group and will be referred to later.

High Lateral Infarction.-The electrocardiograms reproduced in Fig. 29 are those of a man who was under observation off and on from September, 1926, until his death on Jan. 19, 1942. In the autumn of 1934, he began to have mild anginal pain on exertion after meals, and at 3 o'clock on the morning of Aug. 18, 1935, he developed symptoms characteristic of coronary thrombosis. The electrocardiogram taken on the following day presents small QRS deflections, deep $Q$ waves, and sharply inverted $\mathrm{T}$ waves in Lead $\mathrm{I}$. The precordial electrocardiogram taken at the same time, which is not reproduced, is not definitely outside normal limits, but the $R$ and $T$ waves of Leads $V_{1}$ and $V_{2}$ are unusually prominent. The patient had no more anginal pain and got along very well until June 3, 1940, at 12:30 P.M., when a second coronary accident occurred. The electrocardiogram taken two days later is very similar to that of 1935 except that the QRS interval is a little longer. Precordial leads were not used on this occasion. In October, 1941, paroxysmal nocturnal dyspnea began to occur, and in January, 1942, the patient entered the hospital with the symptoms and signs of congestive cardiac failure, and attacks of substernal oppression.

On January 13, the limb leads showed a QRS interval measuring approximately 0.11 second, left axis deviation, and inverted $\mathrm{T}$ deflections in Lead I. The precordial curves of the next day present a very small $R$ deflection in Lead $V_{2}$ and prominent $Q$ waves in Lead $V_{3}$, but no other changes which suggest infarction. The third attack of severe 
anginal pain began at 7 A.M., on January 15. On January 17, right bundle branch block was present. The precordial leads then showed changes characteristic of this conduction defect and small bizarre $Q R S$ deflections in Lead $V_{3}$.

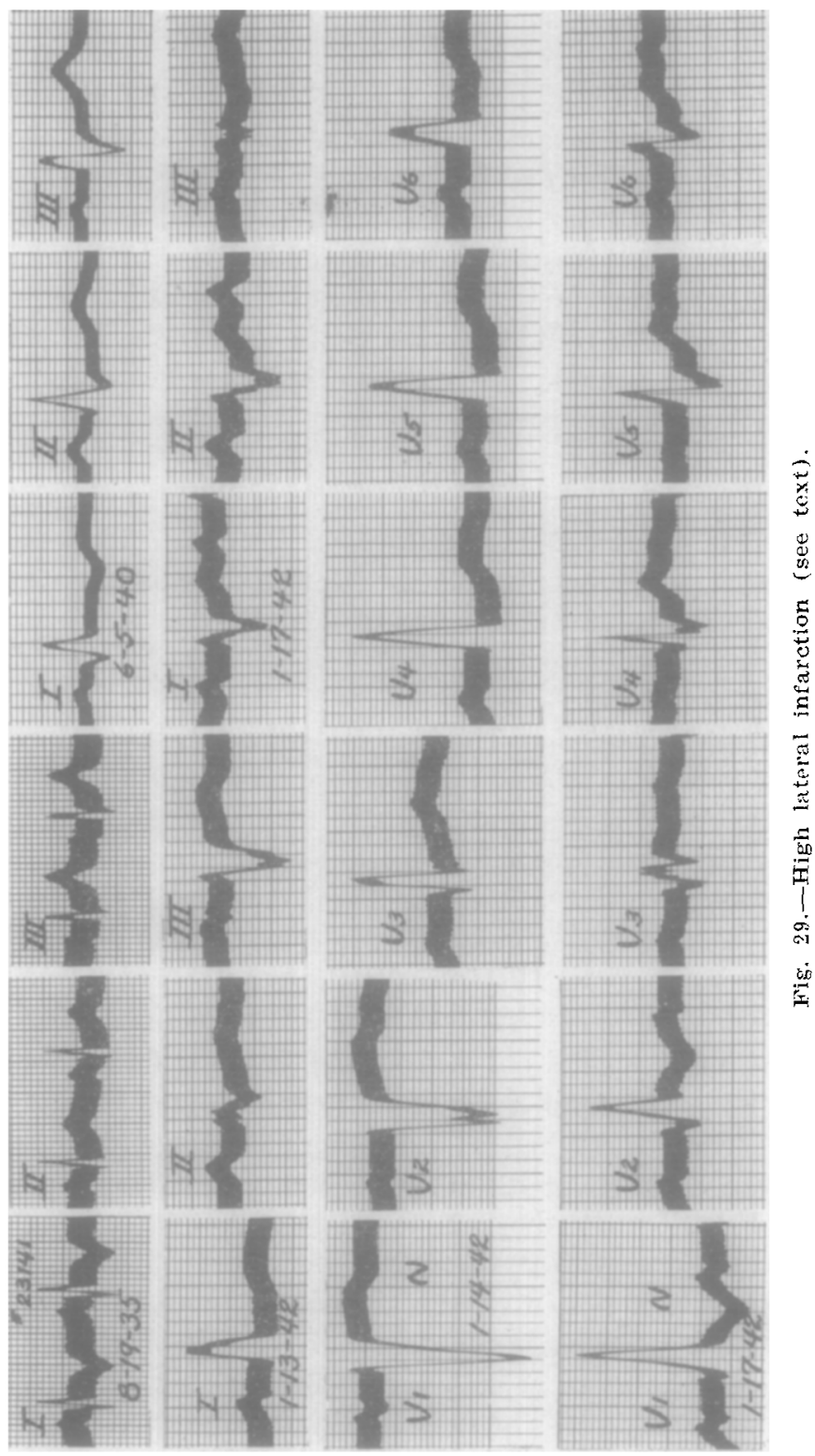

The autopsy diselosed a very large thronbus covering the apical twothirds of the inner aspect of the anterior wall of the left ventricle. Beneath this clot the endocardium was grayish-white in color, and near the base of the ventricle it was about one millimeter thick. Mottled grayish- 
pink to bluish-red areas were scattered through the anterior wall of the left ventricle, the septum, and the apical parts of the right ventricle, which also contained a thrombus. The left coronary artery was blocked by a fresh clot near its origin. In the posterolateral wall of the left ventricle near its base there were extensive grayish-white to grayish-yellow areas which were thought to represent old myocardial infarction. The infarct responsible for the electrocardiographic changes observed in 1935 and 1940 apparently involved this region. We have seen only one other instance in which the limb leads showed signs diagnostic of anterior infaretion and the precordial leads did not.

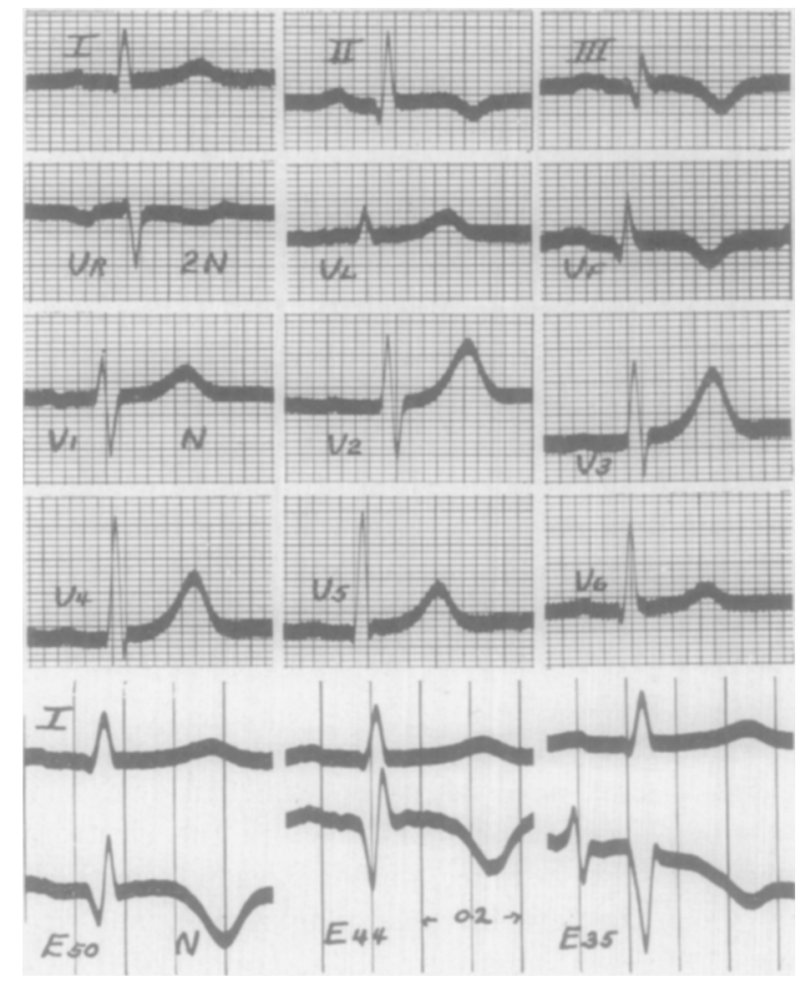

Fig. 30.-Plain posterior infarction. In this a.s in other figures the symbol $E$ is used for unipolar esophageal leads. The flgure which follows this symbol gives the distance (in centimeters) of the esophageal electrode from the nostrils.

Plain Posterior Infarction.--The man, aged 45 years, whose electrocardiograms are shown in Fig. 30, was referred to us for laboratory studies only. He had symptoms typical of coronary thrombosis about one month before these curves were taken. The deep $Q$ waves and sharply inverted $\mathrm{T}$ waves of Leads II and III indicate that there was a recent infarct on the posterior wall of the heart. Note that the potential variations of the outer surface of the infarct were transmitted to the left leg. The precordial leads show no significant abnormalities, but it will be observed that, in Leads $V_{1}$ and $V_{2}$, the $\mathbf{R}$ deflections and $\mathbf{T}$ waves are unusually tall. Characteristic signs of infarction are present 
in the leads from the ventricular levels of the esophagus (Leads $E_{4: 4}$ and $E_{50}$ ). In the lead from the aurieular levels of the esophagus (Lead $E_{35}$ ), there is a large diphasic auricular romplex, and the ventricular complex consists of a large QS deflection followed by an inverted $\mathrm{T}$ wave. Electrocardiograms of this kind are obtained from the auricular levels of the esophagus in normal subjects. ${ }^{21}$ The exploring electrode is close to the auricular wall, which accounts for the character of the auricular complex, and also opposite the great valvular orifiecs at the base of the ventricles so that the negativity of the ventricular cavities is transmitted to it. Large Q or QS deflections in leads from this region have no significance.

In the earliest stages of infarction of the posterior wall of the heart, downward displacement of the RS-T junction is usually present in the precordial leads. In leads from the ventricular levels of the esophagus, the displacement is upward. ${ }^{21}$

Posterolateral Infarction.-The last of the three patients whose electrocardiograms are reproduced in Fig. 28 was a physician, aged 42 years, who had a coronary accident on March 18, 1937. The limb leads, taken on May 25 of the same year, display inverted $T$ waves in Leads I and II and small QRS deflections with prominent $Q$ waves in Leads II and III. There is also a small $Q$ deflection in Lead $I$. The changes in the $T$ waves resemble those found in anterior, and the changes in the QRS complex those found in posterior, infarction. In the precordial curves, the significant abnormalities are in Leads $V_{5}$ and $V_{6}$, which show prominent $Q$ and sharply inverted $T$ waves. It will be noted, however, lhat in the leads from the right side of the precordium, the $\mathrm{R}$ and $\mathrm{T}$ deflections are unusually prominent.

Another electrocardiogram of the same sort is reproduced in Fig. 31. The patient was a man, aged 54 years, who had symptoms typical of coronary thrombosis about one month before the electrocardiographic study was made. In this instance, unipolar leads from the left posterior axillary line, from the left infrascapular region, and from the ventricular levels of the esophagus were taken, and all of these show prominent $Q$ and sharply inverted $T$ waves. Similar but less characteristic changes are present in the leads from the left side of the precordium (Leads $V_{5}$ and $V_{6}$ ). There can be little doubt that the infaret involved the posterolateral wall of the heart. Note that here also large $Q$ and inverted $\mathrm{T}$ waves occur in the unipolar left leg lead. Not all cases in which there are sharply inverted $\mathrm{T}$ waves in Lead $\mathrm{I}$ and abnormally large $Q$ waves in Leads II and III are examples of posterolateral infarction. This electrocardiographic pattern in the limb leads may be produced by the simultaneous presence of two infarcts, an old lesion on the posterior, and a more recent one on the anterior, wall of the heart. It may also occur in anteroseptal infarction in which, perhaps because of some peculiarity in the position of the heart or because there is more 
involvement of the septal wall of the left ventricle than is usual, the potential variations of the central part of the infareted rentricular wall are sometimes transmitted to the left leg.

Posteroinferior Infarction.-The electrocardiograms reproduced in Fig. 32 are those of a man, aged 53 years, who had symptoms typical of coronary ocelusion on Dec. 19, and Dec. 23, 1941. The curves taken

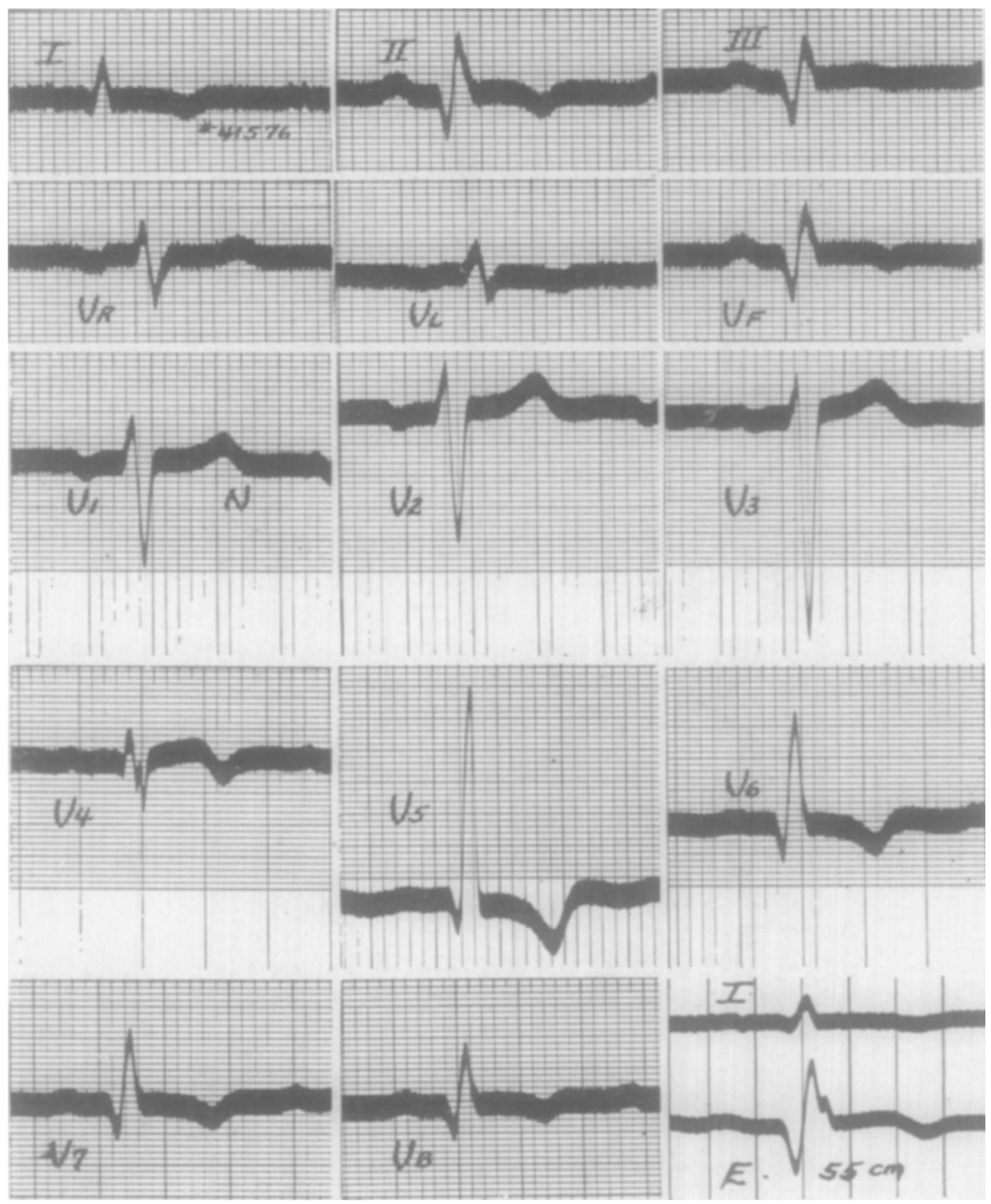

Fig. 31.- Posterolateral infarction.

on December 26 show upward displacement of the RS-T junction, sharply inverted $\mathrm{T}$ waves and prominent $\mathrm{Q}$ deflections in Leads II, III, and $V_{F}$, and complete atrioventricular block, which was transient. Signs diagnostic of infaretion are present in the unipolar lead from the tip of the ensiform cartilage (Lead $V_{E}$ ), but in none of the usual precordial leads. It will be noted that, in this instance, the $R$ and $T$ waves are not unusually prominent in the leads from the right side of the precordium. On the contrary, no $R$ wave is present in Lead $V_{1}$, and the $R$ wave in Lead 
$V_{2}$ is unusually small. In some cases of this sort, slight upward displacement of the RS-T junction occurs ir Lead $V_{1}$ in the early stages of infarction.

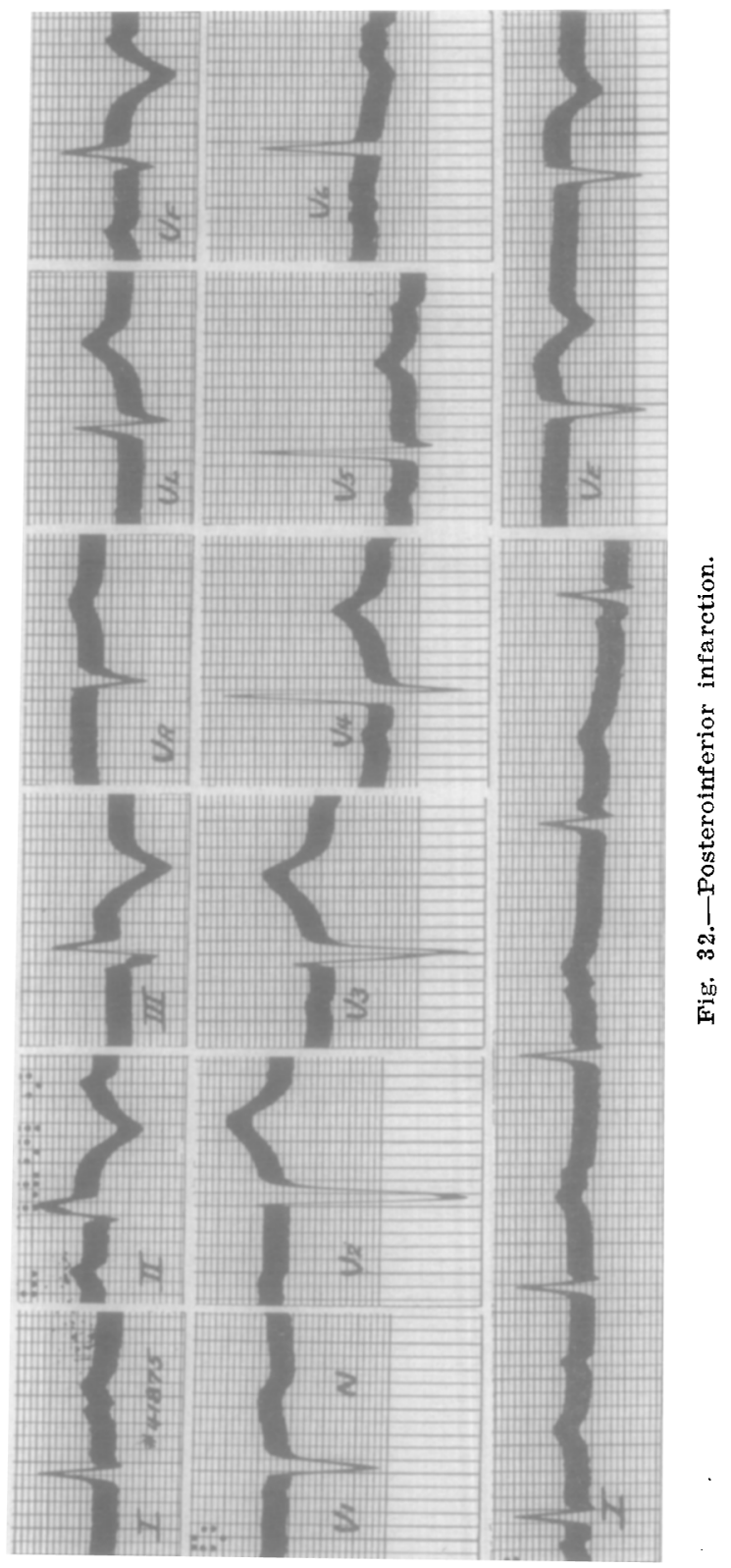

Signs of Posterior Infarction in Limb Leads and of Anterior Infarction in the Precordial Leads.-We have seen only one case of the kind illustrated in Fig. 33. The patient, a man aged 68 years, had a coronary accident on March 31, 1935, while he was unde treatment for prostatic hypertrophy. He died three days later, but permission for an antopsy 
could not be obtained. No symptoms referable to the heart had oceurred previous to this fatal illness. The limb leads show pronounced upward displacement of the RS-T junction and prominent $Q$ deflections in Leads II and III and in precordial Lead $V_{3}$. 'There is, in addition, conspicuous RS-T displacement without characteristic alterations of the QRS complex in Leads $V_{2}$ and $V_{5}$. Leads $V_{4}$ and $V_{8}$ were not taken.

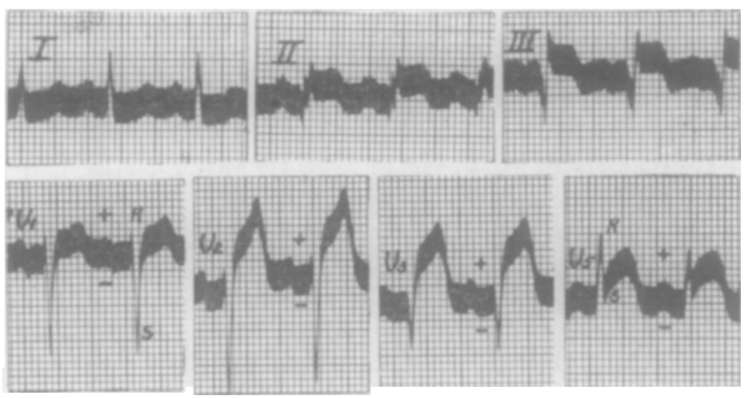

Fig. 33.- Signs of posterior infarction in the limb leads and of anterior infarction in the precordial leads. (After Wilson. ${ }^{22}$ Reproduced with the permission of the Macmillan Company, New York.)

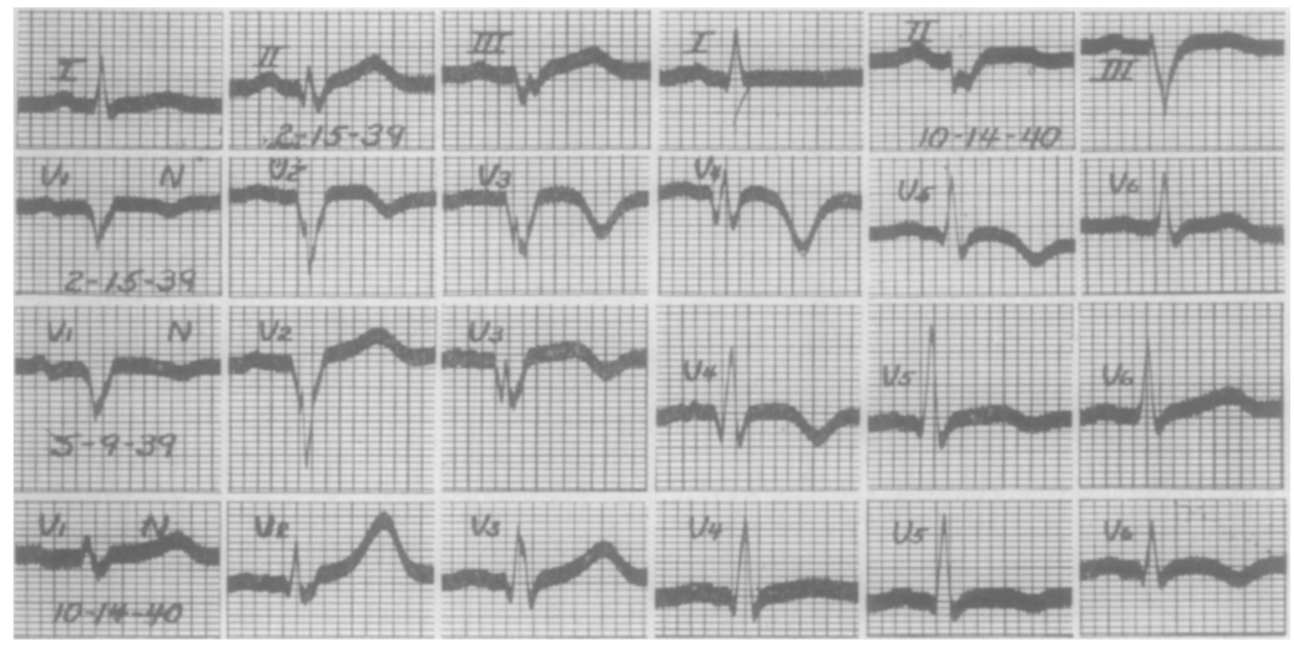

Fig. 34.-Anteroseptal followed by posterolateral infarction.

The character of the electrocardiographic ehanges precludes the possibility that there were two infarcts differing in age. Nor does it appear consistent with the presence of a single infaret extending from the posterior wall of the heart around the left margin onto the anterior wall, for the most striking changes in the precordial leads are in the middle of the series. We are not able to explain these findings satisfactorily, but may point out that apical infarction might give rise to electroeardiographic changes of this sort if the heart were in the vertical position. 
old Anteroseptal and Recent Posterolaterul Infarction.-The electrocardiograms reproduced in Fig. 34 are those of a man, aged 52 years, who had symptoms characteristic of infarction on Dee. 18, 1938, and

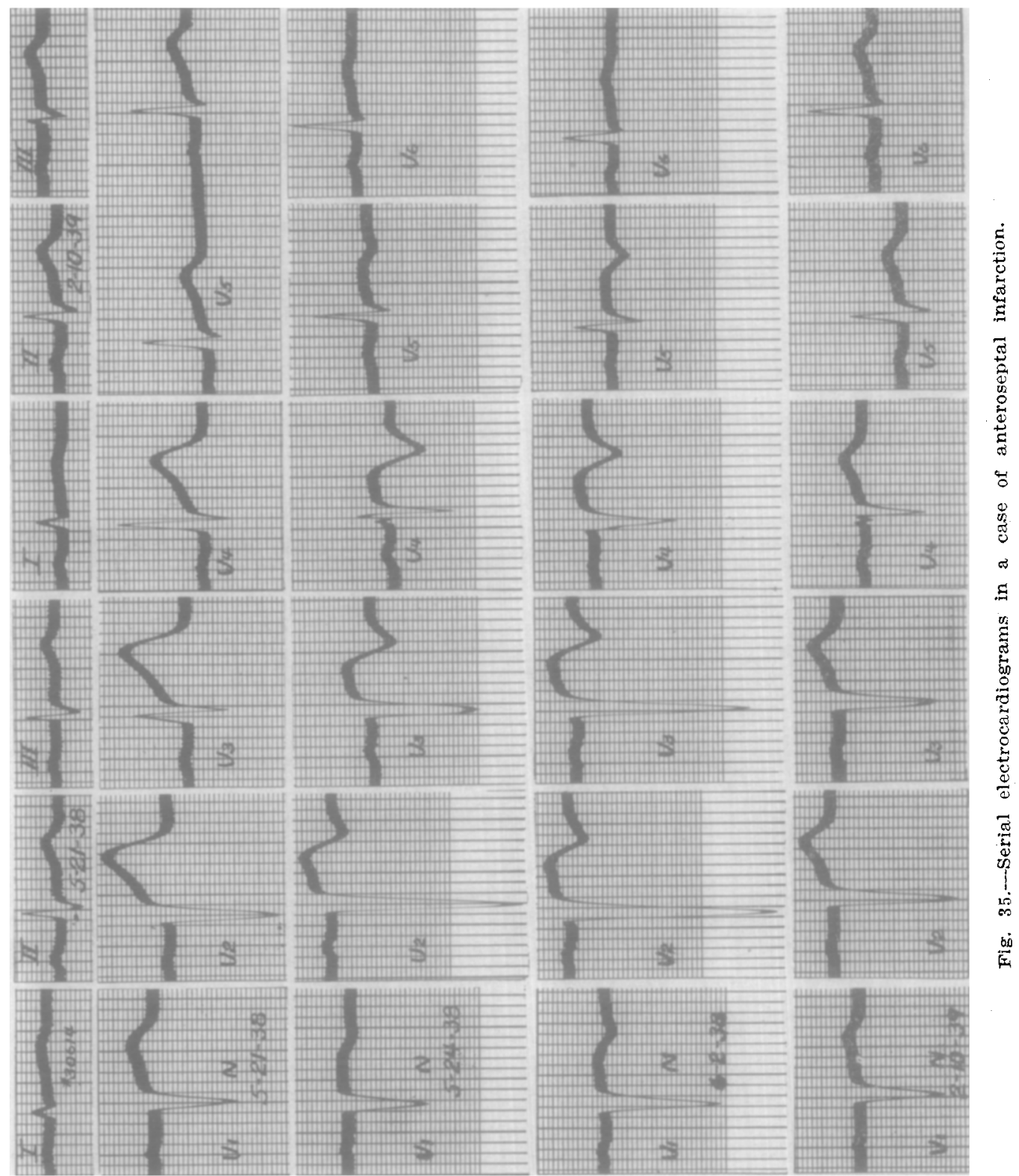

made an uneventful recovery. On Aug. 29, 1940, he had a second attack of severe anginal pain. On this occasion he was examined at his home and the cardioscope disclosed very pronounced upward RS-T displacement in Leads II and III. 
The clectrocardiograms taken two months after the first attack show prominent $Q$ deflections but no charaeteristic T-wave changes in Leads II and III. The precordial curves of the same date display signs diagnostic of infaretion in Leads $V_{2}, V_{3}$, and $V_{4}$, and sharply inverted $T$ waves in Lead $V_{5}$. Note that the ventricular complexes of Lead I resemble those of Lead $\nabla_{6}$ which are in no way abnormal. The infaret was, then, of the anteroseptal variety. We have already mentioned that infarcts of this kind may give rise to prominent $\mathrm{Q}$ deflections in Leads II and III. After the second infarction, sharply inverted $T$ waves appeared in the complexes of Lead $V_{6}$ which had previously eseaped modification, and initial $\mathrm{R}$ deflections were present in Leads II and III. At the same time, conspicuous $\mathbf{R}$ waves were found in the leads from the right side of the precordium in which only embryonic $R$ deflections had previously been present. Note, however, that the resurgent $\mathbf{R}$ waves are preceded by $Q$ deflections in Leads $V_{2}, V_{3}$, and $V_{4}$. Observe also the large size of the $\mathrm{T}$ waves in Leads $V_{1}, V_{2}$, and $V_{3}$, in which deeply inverted $\mathrm{T}$ waves were present after the first coronary accident. The second infarct was, then, of the postcrolateral kind.

We were able to make a more detailed study of another case in which the same series of events occurred. The patient was a man, aged 48 years, who had his first coronary thrombosis on May 21, 1938. The first electrocardiogram was taken about two hours after the onset of characteristic symptoms (Fig. 35). The limb leads showed no significant changes, but there was very pronounced upward displacement of the RS-T junction in Leads $V_{2}, V_{3}$, and $V_{4}$. Three days later, the $R$ deflections had disappeared in Leads $V_{1}, V_{2}$, and $V_{3}$ and had become very small in Lead $V_{4}$, the RS-T displacement had begun to subside, and inversion of the terminal part of the $\mathrm{T}$ waves had developed in Leads $V_{1}, V_{2}, V_{3}, V_{4}$, and $V_{5}$. The ventricular complexes of Lead $V_{6}$ were not affected. In the course of the next year the T deflections regained their normal form, but the QRS changes persisted.

On March 5, 1942, the patient, who had been well for nearly three years, began to have new attacks of severe anginal pain. He was admitted to the hospital, but at first the electrocardiogram showed only the residual changes which dated from his first infarction (Fig. 36). On March 11, however, he developed clear signs of posterior infarction. In the extremity curves, these consisted of pronounced upward displacement of the RS-T junction and prominent Q deflections in Leads II and III. In the precordial electrocardiogram, inverted $\mathbf{T}$ waves eventually developed in the complexes of Lead $V_{6}$, which had escaped in the first attack, and small $\mathrm{R}$ waves appeared in Leads $V_{1}, V_{2}$, and $V_{3}$. In these same leads the $\mathrm{T}$ waves became larger than they had been before.

Anterior Infarction Plus Right Bundle Branch Block.-When anterior infaretion produces right bundle branch block, modifications of the electrocardiogram that can be regarded as diagnostic of infaretion are relatively rare in the limb leads. Displacement of the RS-T junction and 
more or less characteristic changes in the $\mathrm{T}$ waves may be present, but, when the area of the QRS complex is large, they are often obscured by the alterations in the $\mathrm{T}$ complex due to the block. In the precordial leads, on the other hand, entirely characteristic modifications of the QRS

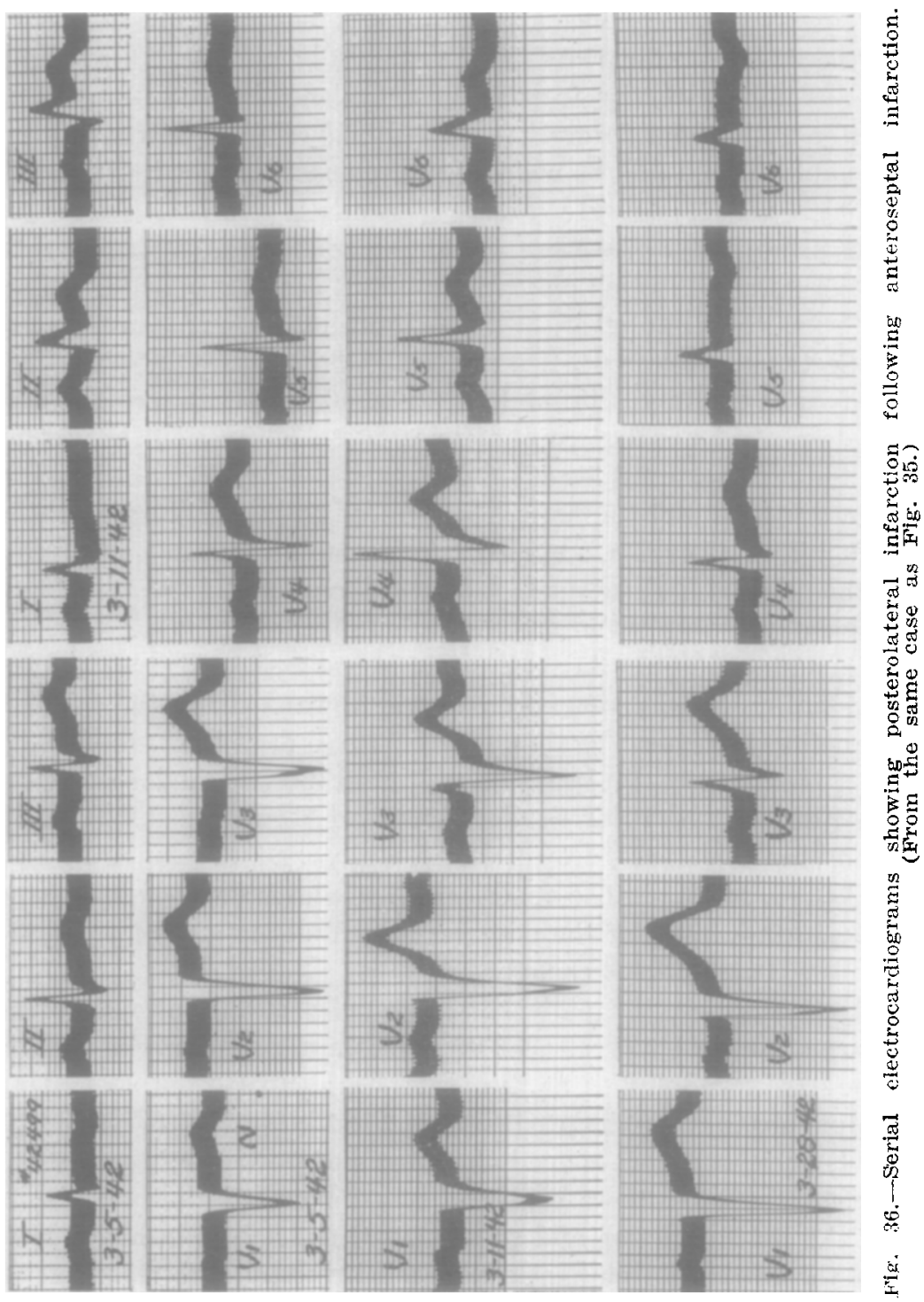

and $\mathrm{T}$ deflections are usually present. We have studied this problem experimentally by producing anterior infaretion and right bundle branch block in dogs, and there are no essential differences between this animal and man, either as regards the character of the changes in the ventricular complexes of the limb leads, or as regards the changes of the ventricular deflections of precordial leads. 
The electrocardiograms reproduced in Fig. 37 are those of a man, aged 37 years, who had severe attacks of anginal pain on July 26 and $28,1938$. Two days after the last attack, a perieardial friction rub was heard. The patient died on Sept. 18, 1938, and autopsy disclosed thrombosis of the anterior descending coronary artery and a very large anterior infaret over which the pericardium had become adherent. Liquefaction neerosis of the central part of the infarct had taken place.

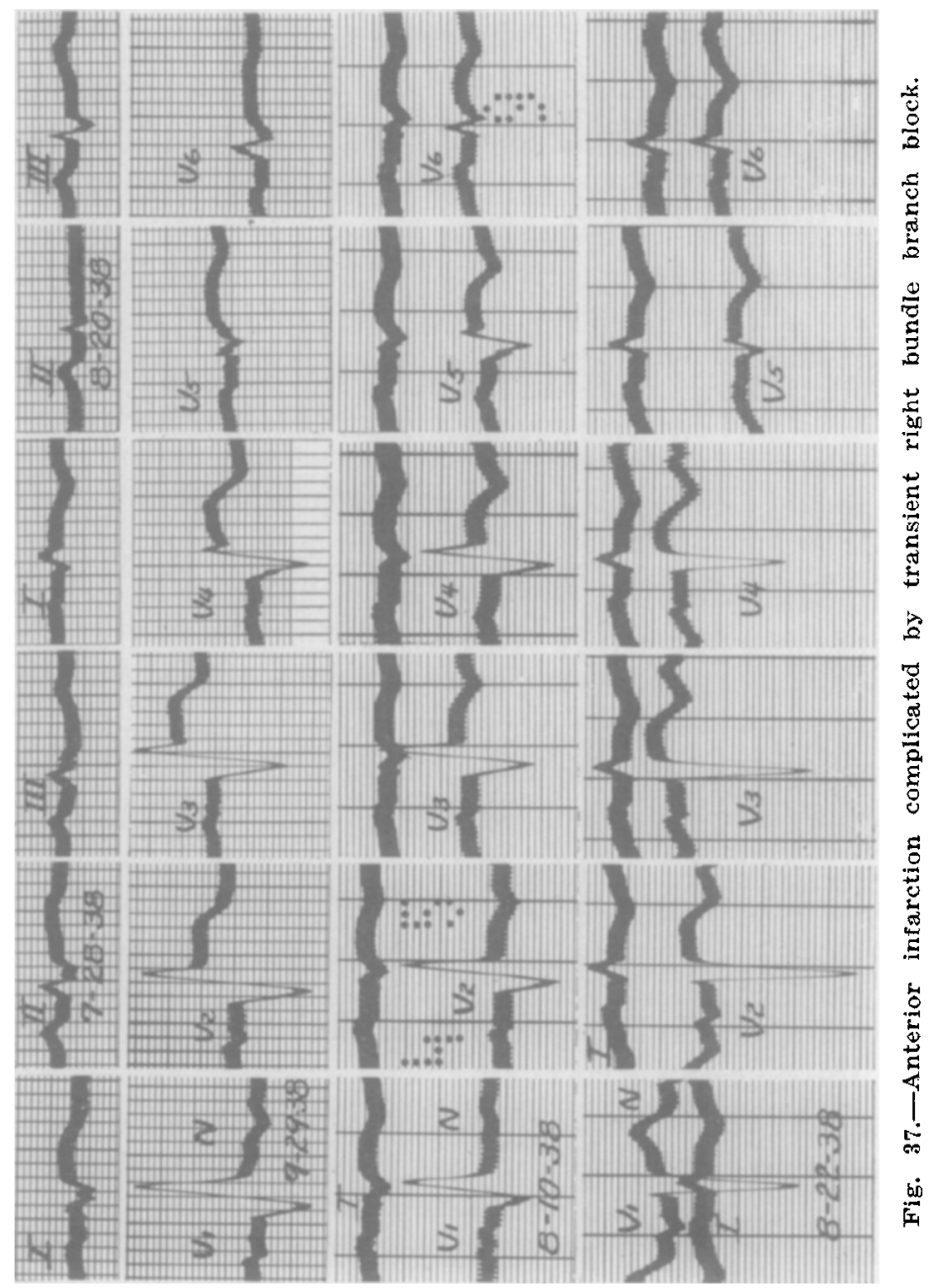

The limb leads, taken on July 28, show small broad QRS complexes and slight upward displacement of the RS-T junction in Lead I. The QRS interval measures approximately 0.12 second. The precordial curves taken on the following day are characteristie of anterior infaretion complicated by right bundle branch block. The presence of the block is indicated by the length of the QRS interval, the very late $R$ waves in Leads $V_{1}, V_{2}$, and $V_{3}$, and the broad $\mathrm{S}$ waves in Lead $V_{6}$. The small 
initial $\mathrm{R}$ deflections which vecur in the leads from the right side of the precordium in uncomplicated right bundle branch block are absent. The presence of the infaret is indicated by the very large $Q$ deflections in Leads $V_{1}, V_{2}, V_{3}$, and $V_{1}$, the small bizarre QRS deflections in Lead $V_{5}$, and pronounced upward displacement of the RS-T junction in Leads $V_{2}, V_{3}$, and $V_{4}$. The precordial curves, taken on August 10, are not essentially different in general outline, but the RS-T displacement is not so great and the end of the $\mathrm{T}$ wave is slightly inverted in Leads $V_{2}$ to $V_{6}$ inclusive.

The electrocardiograms, taken on August 22, are very different. The QRS interval is normal, and small QRS deflections, prominent $\mathrm{Q}$ waves, and sharply inverted $\mathrm{T}$ waves are present in Lead $\mathrm{I}$. In the precordial leads, the $\mathrm{R}$ deflection decreases from Lead $V_{1}$ to Lead $V_{4}$ where it is absent, and there are abnormally large $Q$ deflections in Lead $V_{5}$. Displacement of the RS-T junction and inversion of the end of T are present in Ieads $V_{2}$ to $V_{6}$. It is noteworthy that, although initial $\mathrm{R}$ waves were not present in Leads $V_{1}, V_{2}$, and $V_{3}$ so long as the branch block persisted, they appeared when normal intrarentricular conduction was re-established. It should also be noted that the $\mathrm{S}$ waves which follow these small initial $\mathrm{R}$ deflections are much deeper than the $\mathrm{Q}$ waves present in the same leads during block. These initial $R$ waves must evidently be ascribed to forces produced by activation of the free wall of the right ventricle, and the deep $S$ waves that follow them, at least in part, to the activation of septal muscle from the right side.

Another example of anterior infarction plus right bundle branch block is illustrated in Fig. 38 . The patient was a man, aged 56 years, who had a coronary thrombosis, either on Sept. 23 or on Oet. 3, 1938. The first electrocardiogram, taken before he had had anginal pain, is normal. After the coronary accident, the limb leads disclosed the presence of right branch block but showed no changes characteristic of myocardial infarction. The precordial curves, on the other hand, are characteristic of both of these conditions.

Posterior Infarction Plus Right Bundle Branch Block.-The electrocardiograms reproduced in Fig. 39 are those of a man, aged 53 years, who began to have symptoms characteristic of coronary thrombosis at 9 A.M. on April 30, 1941. The electrocardiograms, taken on May 27, 1941, display large $Q$ waves in Leads IT and III, and sharp inversion of the end of $\mathrm{T}$ in the latter. The QRS interval measures about 0.14 second and there is a broad $\mathrm{S}$ wave in Lead $\mathrm{I}$. The precordial electrocardiogram is characteristic of right bundle branch block. The conspicuous $Q$ waves and T-wave changes in Lead $V_{6}$ suggest that the marginal parts of the infarct involved the lateral wall of the left ventricle. In the cases we have observed, QRS changes in the limb leads characteristic of postcrior infarction have not been obscured br the presence of right hrand blokth. 
WILSON ET AL.: PRECORDIAL ELECTROCARDIOGRAM
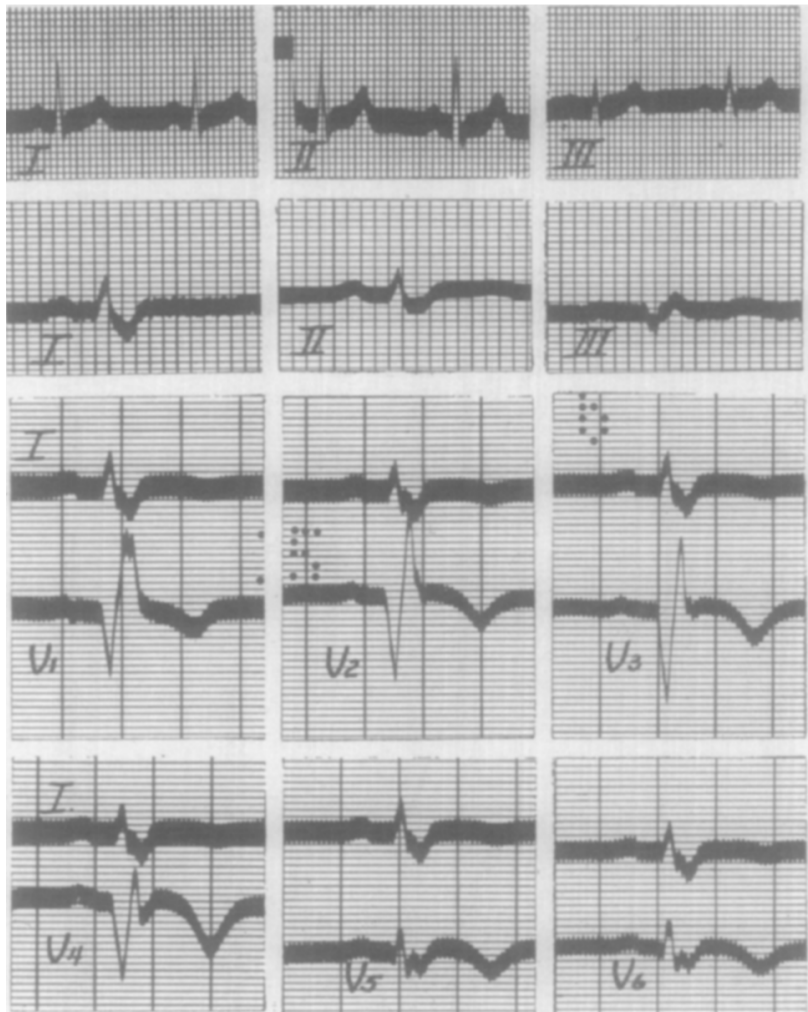

Fig. 38.-Anterior infarction complicated by right bundle branch block.

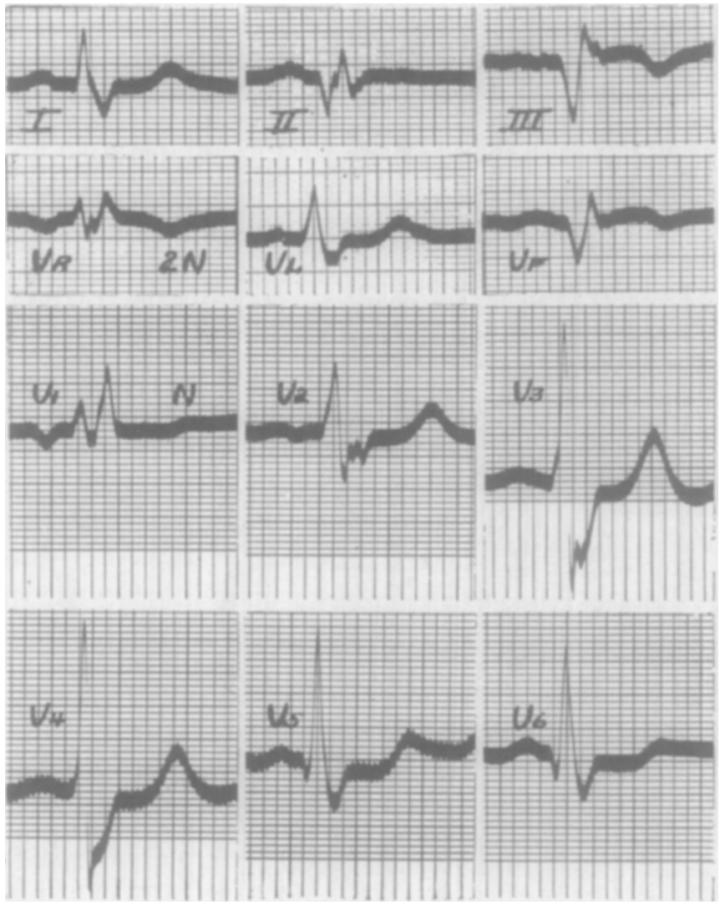

Fig. 39.-Posterior infarction complicated by right bundle branch block. 
Myocardial Infarction Complicated by "Arborization" Block.-The electrocardiograms reproduced in Fig. 40 are those of a man who, after nine years of hypertension, developed myocardial infarction on Aug. 28, 1941. In the electrocardiograms of May 5, 1942, the limb leads display a QRS interval of about 0.14 second, deep, broad $Q$ waves followed $b y$

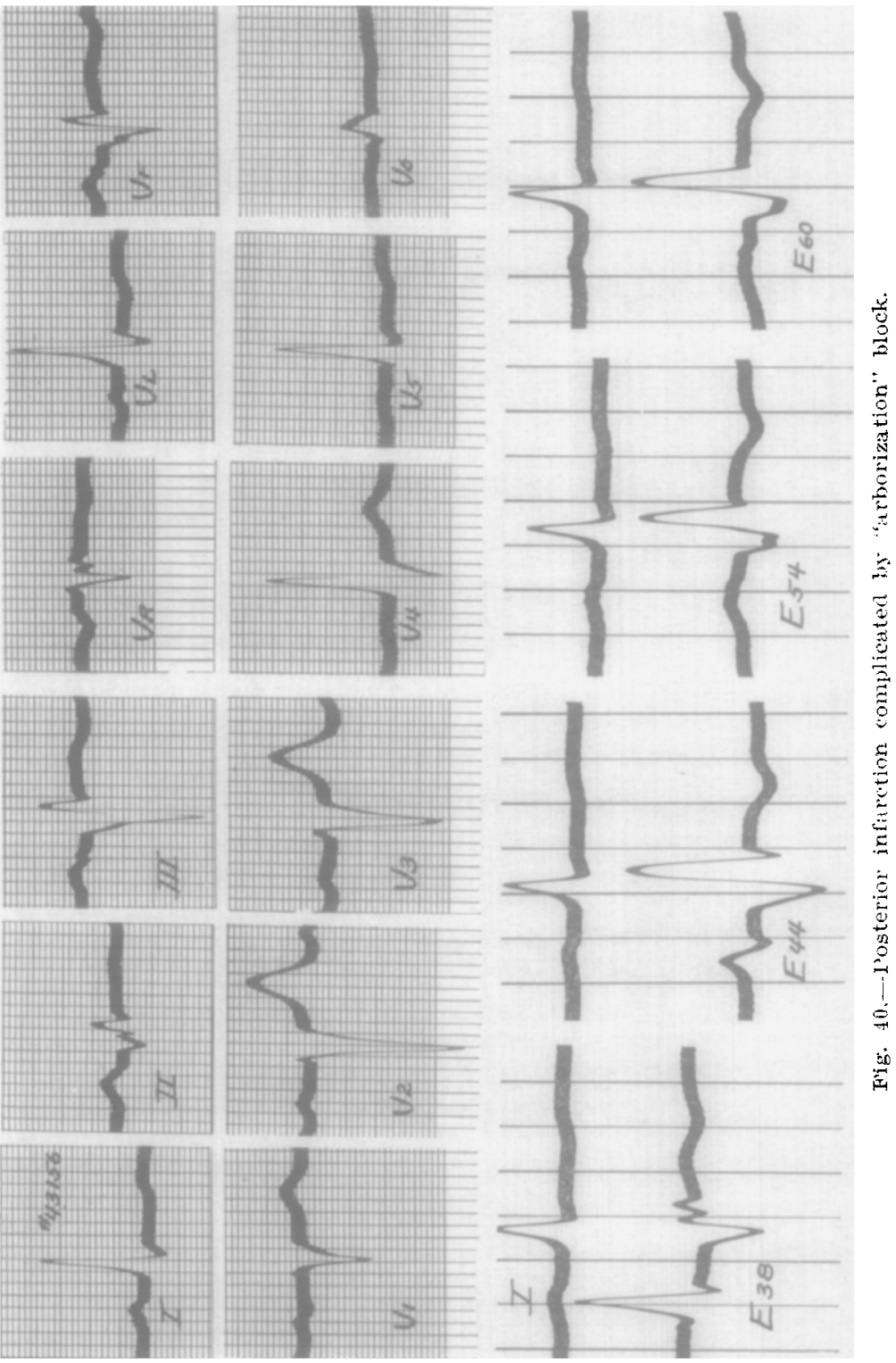

late $\mathrm{R}$ waves in Leads II and III, and conspicuous $\mathrm{S}$ deflections in Lead I. This electrocardingraphie pattern is very similar to that shown in Fig. 39, and posterior infaretion complicated by right branch block was suspected. The precordial electrocardiogram, however, is incompatible 
with the presence of this conduction defect, and shows no changes diagnostic of infarction; nor is it characteristic of left branch block. The leads from the ventricular levels of the esophagus ( $\boldsymbol{E}_{44}, \boldsymbol{E}_{54}$, and $\boldsymbol{E}_{60}$ ) present very broad, notehed $Q$ waves and very late, tall $R$ deflections. It seems probable, therefore, that the infaret involved the subendocardial muscle on the posterior wall of the heart, and not only permitted the negativity of the left ventricular cavity to be transmitted to its epicardial surface during the first part of the QRS interval, but also delayed the activation of less seriously injured outer layers of muscle. which gave rise to the late $\mathrm{R}$ deflection.

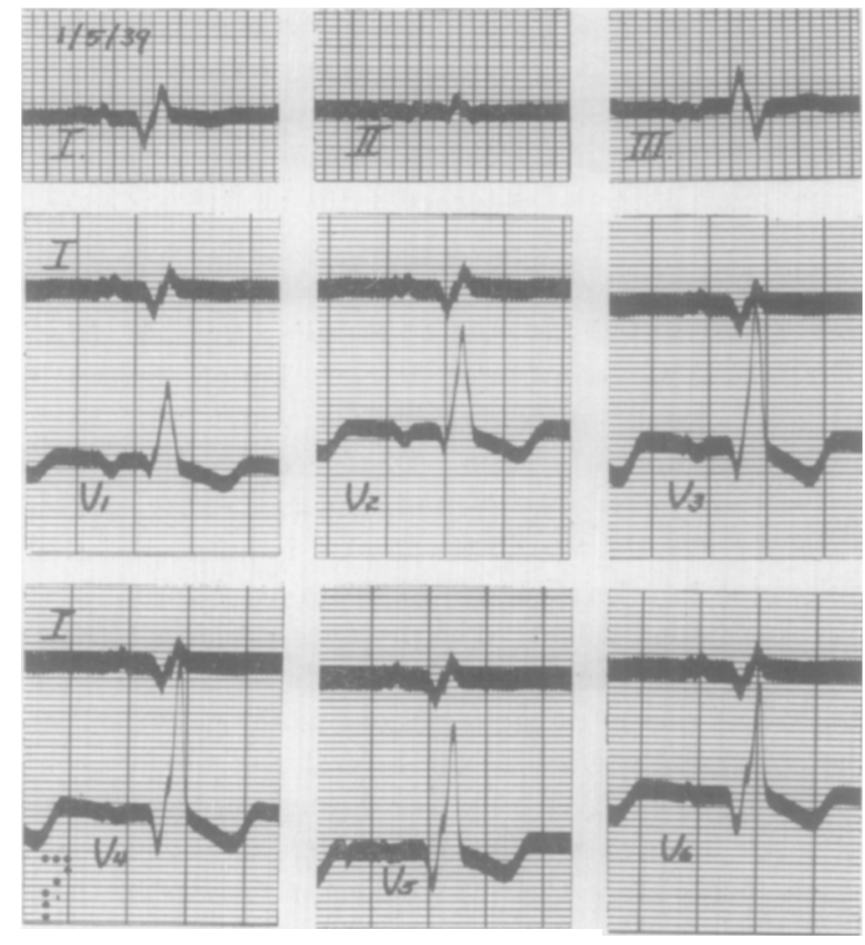

Fig. 41.-Anterior infarction complicated by "arborization block." by right bundle branch block, or by both.

The electrocardiograms reproduced in Fig. 41 are those of a man, aged 45 years, who had symptoms typical of coronary thrombosis, in January, 1938. The limb leads, taken a year later when the patient had congestive cardiac failure, display a $Q R S$ interval measuring between 0.11 and 0.12 second, small QRS deflections, and deep, broad $Q$ waves in Lead I. The curves from the right side of the precordium are like those seen in right bundle branch block complicated by anterior infarction, but in this instance the curves from the left side of the precordium are of the same type. It will be noted that these ventricular complexes closely resemble the ventricular complexes of the esophageal electrocardiograms 
reproduced in Fig. 40. In the case under consideration, leads from the ventricular levels of the esophagus yielded curves of the opposite type; the QRS complex of these tracings is diphasic and consists of an $R$ wave followed by an $\mathrm{S}$ deflection of approximately equal size. It may be that these electrocardiograms represent right bundle branch block complicated by anterior infarction in which there is extreme displacement of the transitional zone. They are, however, more suggestive of anterior infarction with involvement of the subendocardial muscle over a large area, complicated by delayed activation of more normal subepicardial muscle and, perhaps, by right branch block as well. It seems probable that, in some cases of old infarction, the Purkinje network is damaged locally by subendocardial sclerosis and that, as a result, there is a delay in the activation of such living muscle as may remain in the part of the ventricular wall affected. Oppenheimer and Rothschild ${ }^{16}$ had such lesions in mind when they introduced the term "arborization" block, but we doubt very much that the eriteria for the electrocardiographic diagnosis of this condition advanced by them are capable of distinguishing between bundle branch block and intraventricular block of other types.

Infarction Complicated by Left Bundle Branch Block.-When coronary thrombosis is complicated by left bundle branch block, the electrocardiogram rarely presents changes which can be considered diagnostic of myocardial infarction. Displacement of the RS-T segment and changes in the $T$ wave may occur if the area of the QRS complex is small. When the area of the QRS complex is large, the alterations in the $\mathrm{T}$ complex due to infarction are likely to be obscured by those produced by the conduction defect. The presence of characteristic modifications of the QRS deflections in infarction almost always depends upon the transmission of the potential variations of the cavity of the left ventricle to the epicardial surface of the infarct and the adjacent parts of the body. When this cavity is negative throughout the QRS interval, as is the case when intraventricular conduction is normal and in right bundle branch block, large $Q$ or $Q S$ deflections occur in those leads in which one electrode is placed on that side of the left ventricle on which the infaret lies, provided that the connections are made in such a way that negativity of this electrode is represented by a downward deflection. In left bundle branch block, the cavity of the left ventricle is positive at the beginning of the QRS interval, and, consequently, $Q$ or $Q S$ waves do not oceur in leads of the kind mentioned. Direct or semidirect leads from the infarcted wall or from the cavity of this chamber display diphasic QRS complexes consisting of an $\mathbf{R}$ followed by an $\mathrm{S}$ deflection. Ventricular complexes of this sort are not sufficiently distinctive to have diagnostic value. When the septum is infarcted, as well as the free wall of the left ventricle, the cavity of the left ventriele is initially negative because the negativity of the cavity of the right is transmitted to it. Under these circumstances, the electrocardiogram may display large $Q$ 
or QS deflections in leads from the left precordium. The presence of large $Q$ waves in these leads in left bundle branch block should always lead to the suspicion that the ventricular septum is involved.

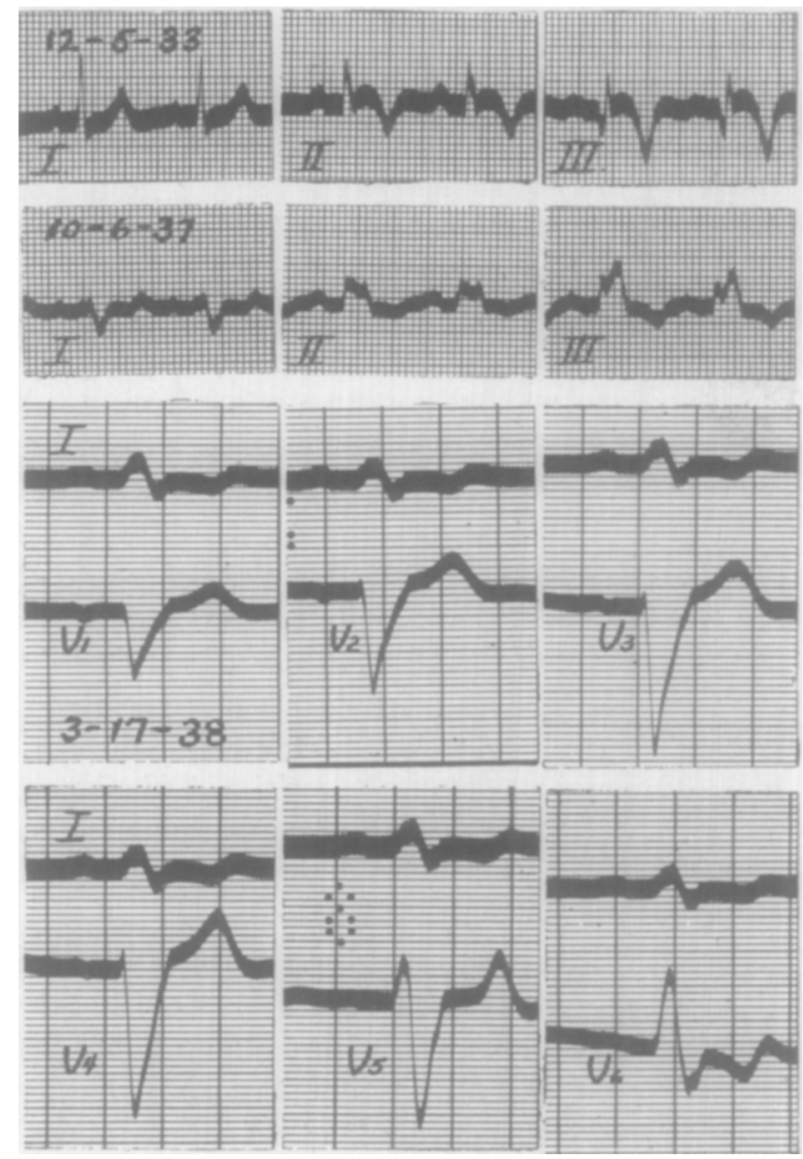

Fig. 42.-Posterior infarction complicated by left bundle branch block.

A case of infarction which was complicated by left bundle branch block is illustrated in Fig. 42. The patient was a man, aged 56 years, who had a coronary occlusion on Nov. 24,1933 . When he was first seen about ten days later, the limb leads showed the changes usually observed in posterior infarction. Except for inversion of the $\mathrm{T}$ waves in Lead $V_{0}$ the precordial leads were negative. A week later, the patient developed transient intraventricular block with ventricular complexes similar to those observed in 1937 and 1938 (see Fig. 42). The area of the QRS group was small, and the block did not abolish the inverted $\mathrm{T}$ waves in Leads II and III. It did abolish the prominent $\mathrm{Q}$ waves in these leads. These curves have been published (Wilson, ${ }^{22}$ Fig. 78). When the patient returned in 1937, the intraventricular block was again present and it persisted. The ventricular complexes of the standard leads are of the 
kind ascribed to right bundle branch block, but the precordial leads indicate that the conduction defect was in the left branch of the His bundle instead of the right. The heart was in the vertical rather than in the horizontal position. The QRS complexes of Lead $V_{8}$ are diphasic and, as regards their gencral outline, of the sort obtained by leading directly from the surface of an infarct of the left ventricular wall when left branch block is present. But QRS deflections of this kind may occur in Lead $V_{\mathrm{a}}$ merely because the transitional zone is shifted unusually far to the left. They are not sufficiently distinctive to be helpful in the diagnosis of infarction. A case of septal infaretion plus left bundle branch block is described in the article to which we have already referred (see Fig. 82 of that article).

In conclusion, we may say that we feel certain that, in the long run, unipolar precordial leads will be found superior to precordial leads of other kinds. They are the best available substitute for unipolar direet leads from the anterior surfaces of the ventricles. They are not directly influenced by the potential variations of the extremities; this makes them valuable in the analysis of electrocardiographie patterns in the standard limb leads with which we have long been familiar.

We must add that many of the views expressed here must be regarded as tentative. There are still many problems to be solved. We do not know exactly what degree of correlation may exist between the electrocardiographic position and the anatomic position of the heart. We have, as yet, very little information as to the effect of the latter upon the precordial electrocardingram. The factors that determine the location and breadth of the transitional zone are still obscure. It is desirable that the effects of preponderant hypertrophy of the right and of the left ventricle upon the form of the precordial deflections be studied experimentally. It is still impossible to diagnose the lesser grades of this condition with any certainty. We need to have a better idea as to what parts of the heart's surface play the most important role in determining the potential variations of the extremities and other points on the body distant from the heart. We must study more carefully the electrocardiographic changes produced by combinations of lesions, such as right ventricular hypertrophy and right bundle branch block, and by conduction defects which delay the activation of local regions of muscle, particularly in myocardial infarction ("arborization block"). We must determine more exactly what the different electrocardiographic patterns seen in infarction mean in terms of the size of the infarct, its location, and the character of the changes in the muscle involved.

We feel sure that the future will see a great increase in the use of precordial and other special leads, and that it will bring us far greater knowledge of the electrocardiogram than wo now possess.

\section{RFFFRFNCFS}

1. Lewis, T., and Rothschild, M. A.: The Excitatory Process in the Dog's Heart. Part II. The Ventricles, Phil. Trans. Roy. Soe. Lond. Series B. 206: 181, 1915. 
2. Wilson, F. N., Macleod, A. G., and Barker, P. S.: The Distribution of the Action Currents Produced by Heart Muscle and Other Excitable Tissues Immersed in Extensive Conducting Media, J. Gen. Physiol. 16: 423, 1933.

3. Cole, K. S., and Curtis, H. J.: Electric Impedance of Squid Giant Axon During Activity, J. Gen. Physiol. 22: 649, 1939.

4. Wilson, F. N., Hill, I. G. W., and Johnston, F. D.: The Interpretation of the Galvanometric Curves Obtained When One Electrode Is Distant From the Heart and the Other Near or in Contact With Its Surface, Am. HearT J. 10: $176,1934$.

5. Wilson, F. N., Macleod, A. G., and Barker, P. S.: The Potential Variations Produced by the Heart Beat at the Apices of Einthoven's Triangle, Am. Heart J. 7: $207,1931$.

Wilson, F. N., Johnston, F. D., Macleod, A. G., and Barker, P. S.: Electrocardiograms That Represent the Potential Variations of a Single Electrode, AM. HeART J. 9: 447, 1934.

6. Eckey, P., and Fröhlich, R.: Zur Frage der unipolaren Ableitung des Elektrokardiogramms, Arch. f. Kreislaufforseh. 2: 349, 1938.

7. Burger, R.: Ueber die herzferne Elektrode bei der semidirekten Ableitung; über dic Nullpotentialclektrode von Wilson; über die Verwendung einer von der gesamten Körperoberfläche ableitenden Elektrode als indifferente TestElektrode, Cardiologia 3: 56, 1939.

8. Burger, R., and Wuhrmann, F.: Ueber das elektrische Feld des Herzens. 2 Mitteilung; Die Darstellung des elektrischen Feldes des Herzens durch das "Diagramm der Potentialdifferenzen." Der Vergleich dieses Diagramms mit dem Vektordiagramm, Cardiologia 3: 139, 1939.

9. Arrighi, F.: Personal communication.

10. Supplementary Report by the Committee of the American Heart Association for the Standardization of Precordial Leads, AM. HEART J. 15: 235, 1938.

11. Kossmann, C. E., and Johnston, F. D.: The Precordial Flectrocardiogram. I. The Potential Variations of the Precordium and of the Extremities in Normal Subjects, AM. HEART J. 10: 925, 1935.

12. Goldberger, E.: A Simple Indifferent Electrocardiographic Electrode of Zero Potential and a Technique of Obtaining Augmented Unipolar Extremity Leads, AM. HEART J. 23: 483, 1942.

13. Wilson, F. N., Johnston, F. D., Cotrim, N., and Rosenbaum, F. F.: Relations Between the Potential Variations of the Ventricular Surfaces and the Form of the Ventricular Electrocardiogram in Leads From the Precordium and the Extremities, Tr. A. Am. Physicians 56: 258, 1941.

14. Kraus, F., and Nicolai, G.: Das Elektrokardiogramm des gesunden und kranken Menschen, Leipzig, 1910, Veit and Comp.

15. Wilson, F. N.: Concerning the Form of the QRS Deflections of the Electrocardiogram in Bundle Branch Block, J. Mt. Sinai Hosp. 8: 1110, 1942.

16. Oppenheimer, B. S., and Rothsehild, M. A.: Electrocardiographic Changes Associated With Myocardial Infarction, J. A. M. A. 69: 429, 1917.

17. Hecht, H., Johnston, F. D., and Wilson, F. N.: Unpublished observations.

18. Johnston, F. D., Hill, I. G. W., and Wilson, F. N.: The Form of the Electrocardiogram in Experimental Myocardial Infarction. II. The Early Effeets Troduced by Ligation of the Anterior Descending Branch of the Left Coronary Artery, AM. HeART J. 10: 889, 1935.

19. Wilson, F. N., Johnston, F. D., and Hill, I. G. W.: The Form of the Electrocardiogram in Experimental Myocardial Infarction. IV. Additional Observations on the Later Effects Produced by Ligation of the Anterior Descending Braneh of the Left Coronary Artery, AM. HEART J. 10: 1025, 1935.

20. Langendorf, R.: Personal communication to F. F. Rosenbaum.

21. Hamilton, J. G. M., and Nyboer, J.: The Ventricular Deflections in Myocardial Infaretion. An Electrocardiographic Study Using Esophageal and Precordial Leads, Aм. HEART J. 15: 414, 1938.

Nyboer, J.: The Normal and Abnormal Esophageal Electrocardiogram With Particular Reference to Myocardial Infarction, AM. HЕAнT J. 22: 469, 1941.

22. Wilson, F. N.: The Electrocardiogram in Diseases of the Coronary Arteries, Chapter XII in: Levy, R. L.: Diseases of the Coronary Arteries and Cardiae Pain, New York, 1936, The Macmillan Company. 